University of Louisville

ThinkIR: The University of Louisville's Institutional Repository

$12-2013$

\title{
An improved thin-film microelectrode array and signal conditioning board for measuring cardiac surface potentials.
}

Christopher Hayward Osborne

University of Louisville

Follow this and additional works at: https://ir.library.louisville.edu/etd

\section{Recommended Citation}

Osborne, Christopher Hayward, "An improved thin-film microelectrode array and signal conditioning board for measuring cardiac surface potentials." (2013). Electronic Theses and Dissertations. Paper 1084.

https://doi.org/10.18297/etd/1084

This Master's Thesis is brought to you for free and open access by ThinkIR: The University of Louisville's Institutional Repository. It has been accepted for inclusion in Electronic Theses and Dissertations by an authorized administrator of ThinkIR: The University of Louisville's Institutional Repository. This title appears here courtesy of the author, who has retained all other copyrights. For more information, please contact thinkir@louisville.edu. 


\title{
AN IMPROVED THIN-FILM MICROELECTRODE ARRAY AND SIGNAL CONDITIONING BOARD FOR MEASURING CARDIAC SURFACE POTENTIALS
}

\author{
By \\ Christopher Hayward Osborne \\ B.S., University of Louisville, 2010

\begin{abstract}
A Thesis
Submitted to the Faculty of the University of Louisville

J. B. Speed School of Engineering as Partial Fulfillment of the Requirements for the Professional Degree
\end{abstract}

\section{MASTER OF ENGINEERING}

Department of Bioengineering

December 2013 


\section{AN IMPROVED THIN-FILM MICROELECTRODE ARRAY AND SIGNAL CONDITIONING BOARD FOR MEASURING CARDIAC SURFACE POTENTIALS}

Submitted by:

Christopher Hayward Osborne

A Thesis Approved On

December 2, 2013

by the Following Reading and Examination Committee:

Dr. Robert Keynton, Thesis Director

Dr. Steven Koenig

Dr. John Naber 



\section{ACKNOWLEDGEMENTS}

I owe a great deal of thanks to Dr. Huihang Dong. It was only through his patience, kindness, and willingness to teach and give that I was able to accomplish what I have. I have the utmost respect and gratitude toward him for his help.

Similarly, if it were not for the opportunity that Dr. Robert Keynton has given me through a graduate assistantship I would not have been able to accomplish any of this. I am honored and thankful that I was thought of and chosen by him to do this work.

If it were not for the continual support of Mr. Mark Crain I do not feel I would have been successful. He provided technical and emotional support to me that has been invaluable and I cannot express my gratitude enough.

The same is true of Mr. Thomas Roussel and Mr. Scott Cambron. Both have supported me and helped me solve problems when I couldn't find solutions.

I am incredibly grateful for the help of Dr. Derek Dosdall of the University of Utah in providing his lab and his time to perform meaningful experiments using the devices I've built.

More than anyone else, I have my family and my good friends to thank for being able to get to this point. Without their support, in every way, none of this would have been a possibility. I can't express how thankful and blessed I am to have each of you. 


\begin{abstract}
The work outlined in this thesis expanded on previous work to improve a thin-film microfabricated electrode array intended for cardiac electrophysiology studies. A thin layer of silver was added in between titanium and platinum electrical trace layers to reduce electrode resistance. A $200 \mathrm{~nm}$ layer of silver decreased electrode resistance by an order of magnitude. In addition, a new high quality signal conditioning board was developed using precision operational amplifiers from Texas Instruments. Both new sensor and board design were verified together through in vivo studies using rabbit, goat, and dog hearts.
\end{abstract}




\section{TABLE OF CONTENTS}

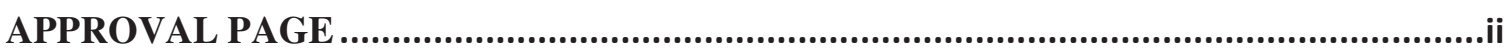

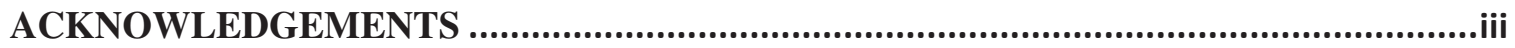

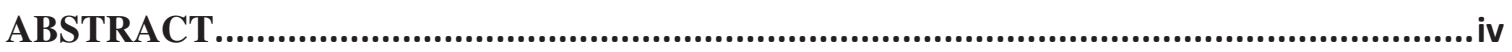

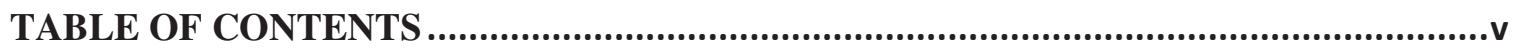

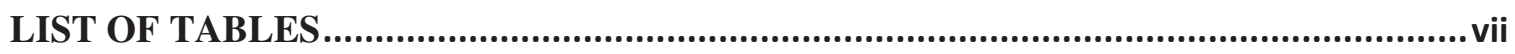

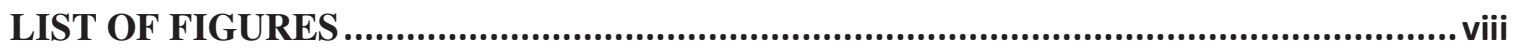

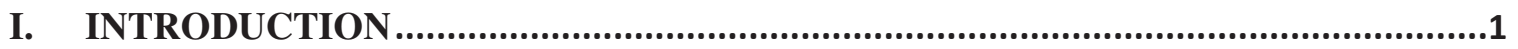

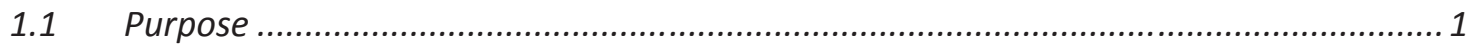

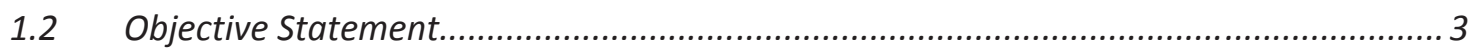

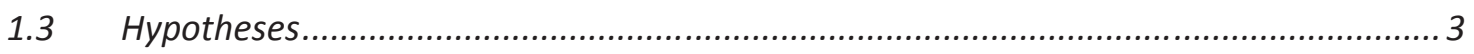

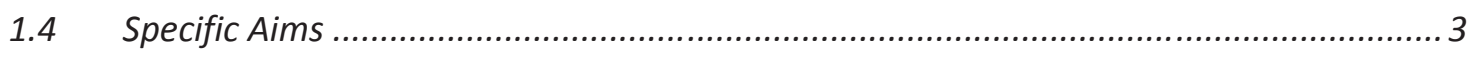

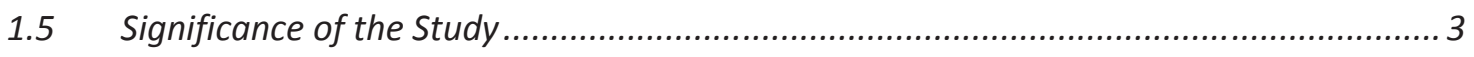

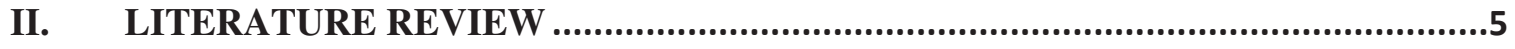

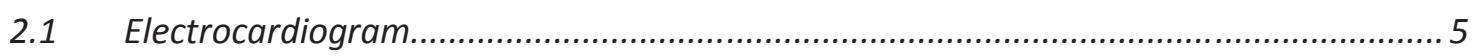

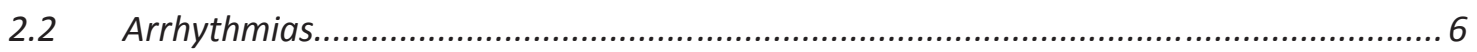

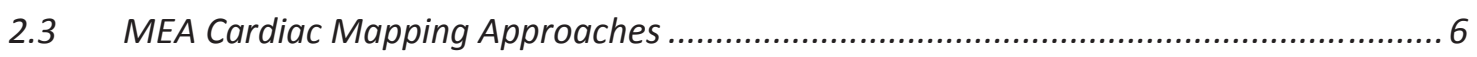

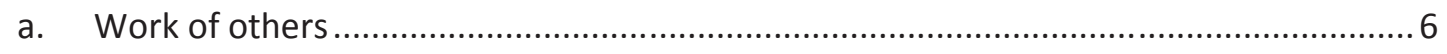

b. University of Louisville TFMEA-SCM system ........................................................... 7

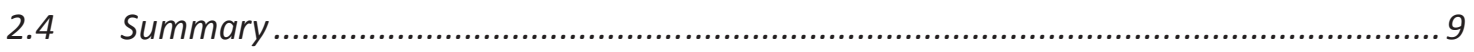

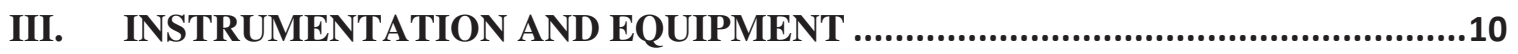

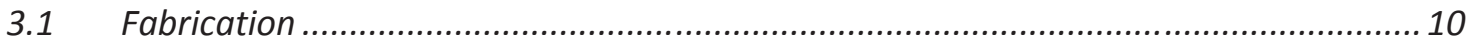

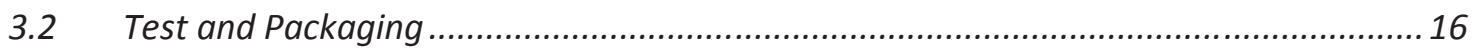

3.3 Signal conditioning board design and testing ........................................................ 17

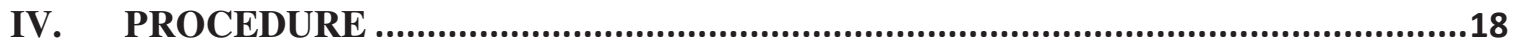

4.1 Brief Overview of Previous Generation System........................................................ 18

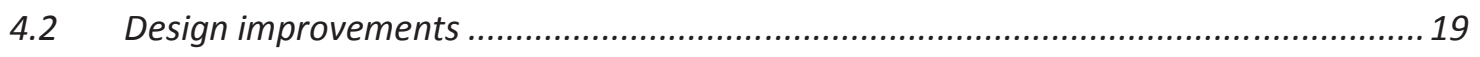

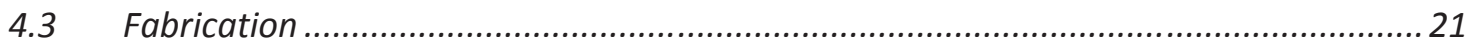

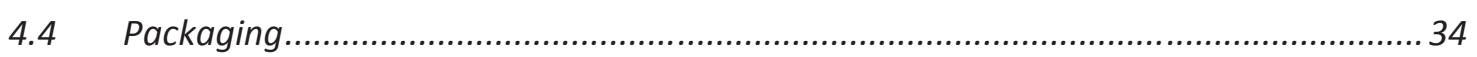

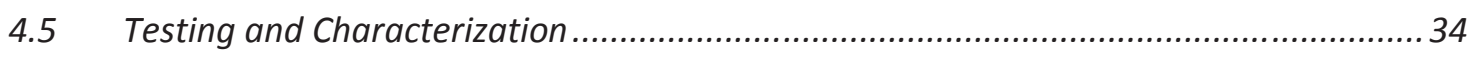

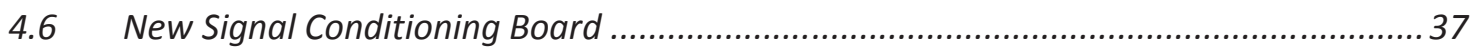

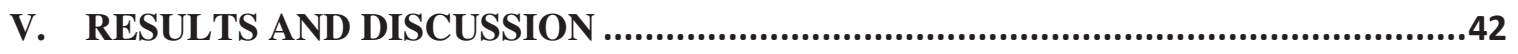

5.1 TFMEA Metrology and Electrical Resistance Measurements....................................... 42

5.2 Preliminary In vitro Characterization of New TFMEA with previous SCM......................44

5.3 Preliminary In vivo Characterization of New TFMEA with previous SCM ..................... 45

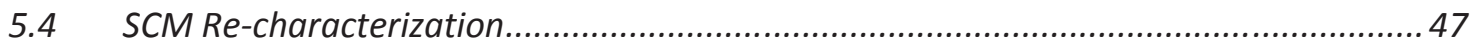

5.5 New Signal Conditioning Board (SCB) Fabrication, Testing and Characterization......... 50 


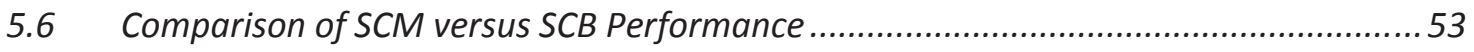

5.7 In vitro characterization of new TFMEA interfaced to new SCB ..................................5

5.8 In vivo characterization of new MEAs interfaced to new SCB....................................58

VI. CONCLUSION AND RECOMMENDATION ..................................................65

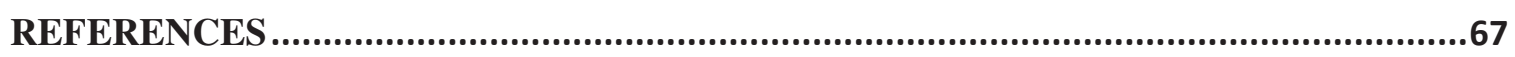

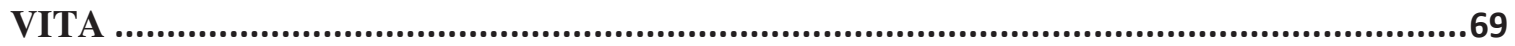




\section{LIST OF TABLES}

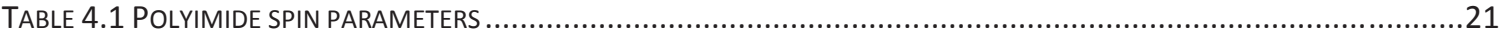

TABLE 4.2 POLYIMIDE CURING PARAMETERS.. 


\section{LIST OF FIGURES}

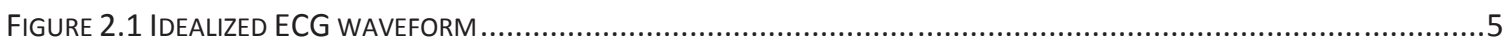

FIGURE 2.2 LEFT, INTER-ELECTRODE SPACING IS SHOWN TO BE $75 \mu \mathrm{M}$ IN EACH GROUP, DIAMETER OF EACH ELECTRODE IS $25 \mu \mathrm{M}$.

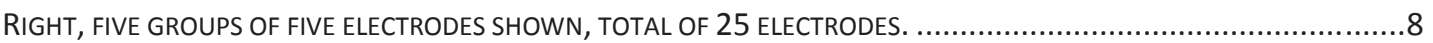

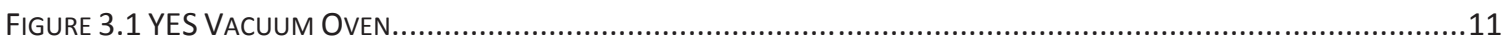

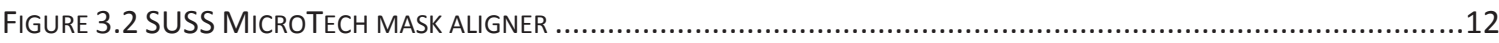

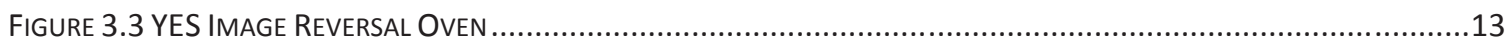

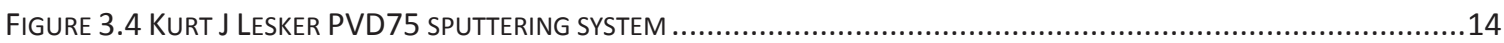

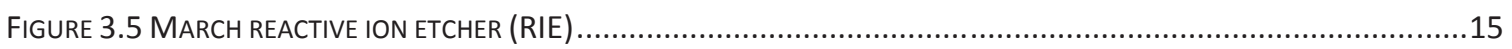

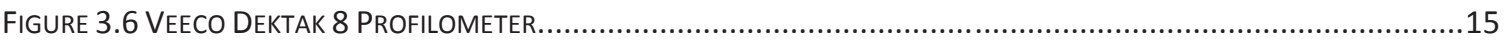

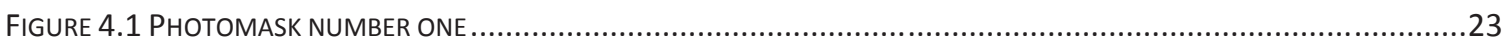

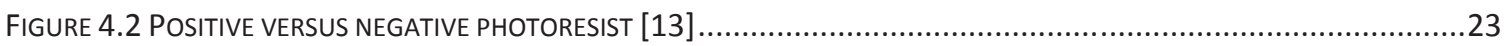

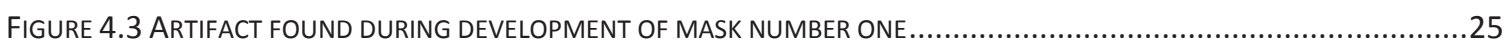

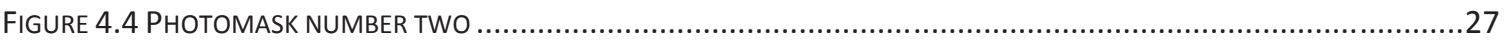

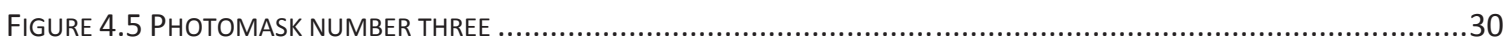

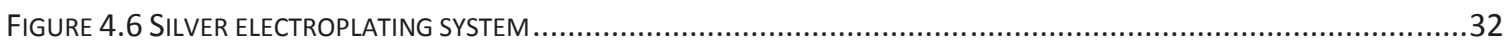

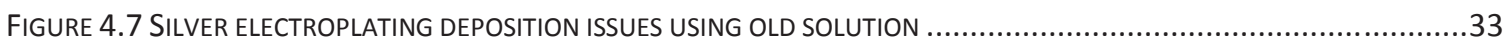

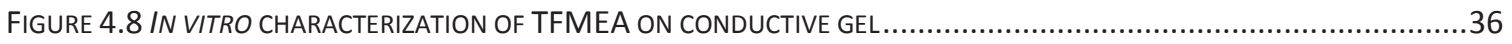

FIGURE 4.9 ONE GROUP OF FIVE ELECTRODES, SHOWING THE CENTER, NORTH, SOUTH, EAST, AND WEST PADS .....................37

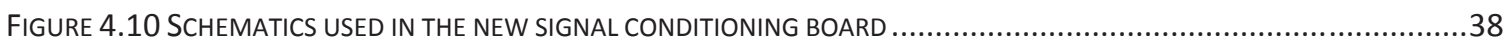

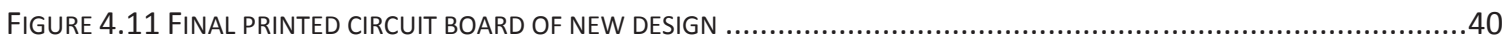

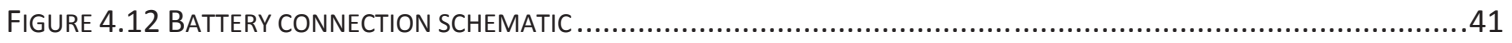

FIGURE 5.1 IN VITRO GEL CHARACTERIZATION OF IMPROVED TFMEA WITH SCM, ELECTRICAL NOISE SHOWN BELOW............45

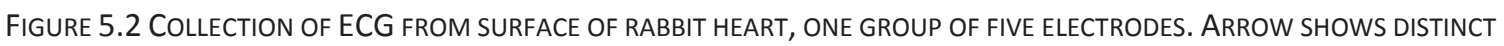

JUMP.

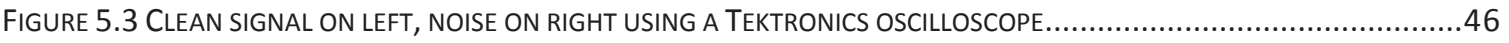

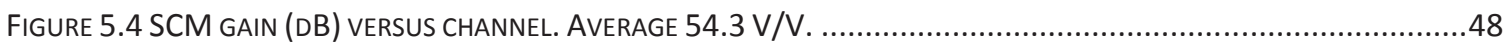

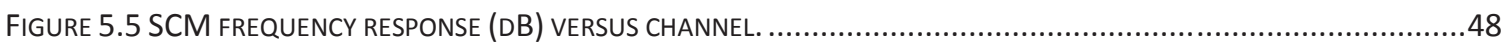

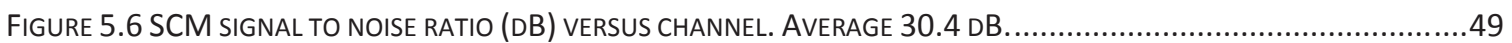

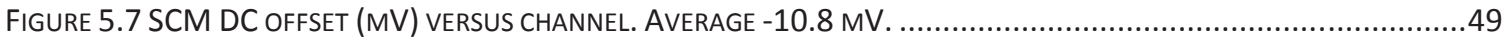

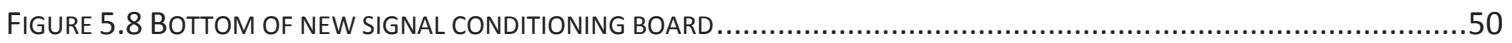

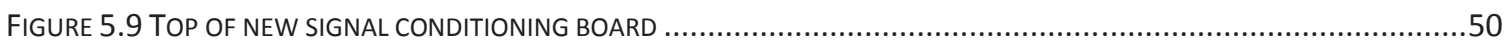

FIGURE 5.10 GAIN MEASUREMENTS FOR ALL CHANNELS ACQUIRED FOR NEW SCBS ................................................5

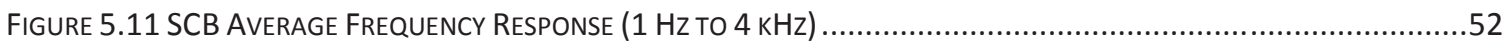

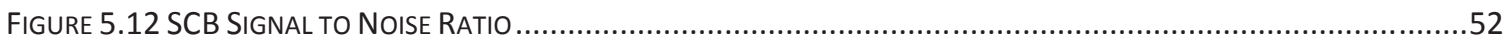

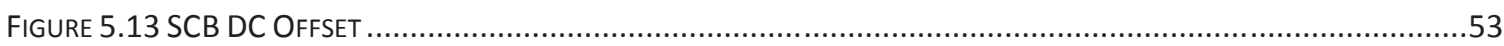

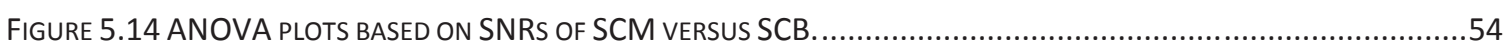

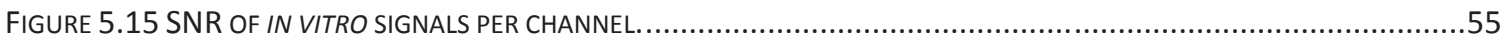

FIGURE 5.16 ONE GROUP OF FIVE ELECTRODES USING IMPROVED TFMEA AND SCM. .........................................56

FIGURE 5.17 ONE GROUP OF FIVE ELECTRODES USING IMPROVED TFMEA AND SCB. ...........................................56

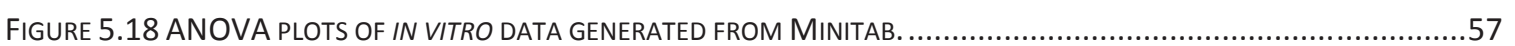

FIGURE 5.19 FFT FROM RIGHT VENTRICLE DATA COLLECTED IN A DOG, 0 TO 1.5 kHz. SIGNAL LEVEL -40DB........................59

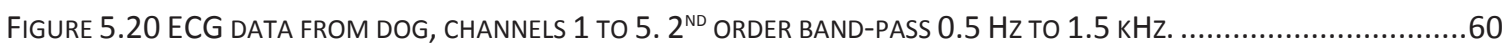

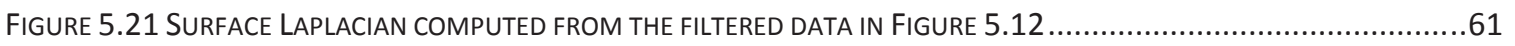


FiguRE 5.22 FFT FROM LEFT VENTRICLE DATA COLLECTED IN A GOAT RUN 2, 0 TO 1.5 kHz. SignAL LEVEL -20 dB.............62

FIGURE 5.23 ECG FROM GOAT LEFT VENTRICLE RUN 2, CHANNELS 1 TO 5. $2^{\text {ND }}$ ORDER BAND-PASS 0.5 HZ TO 1.5 KHZ.........63

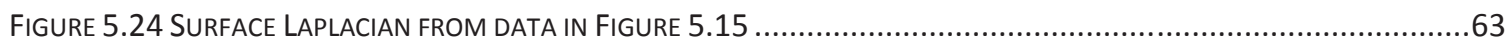





\section{INTRODUCTION}

\subsection{Purpose}

Each year nearly 360,000 Americans die as a result of out-of-hospital sudden cardiac arrest (SCA) [1]. During SCA the normal rhythm or beat of the heart is disrupted and becomes erratic, which stops the heart from circulating blood. This disruption is called an arrhythmia. The electrical events that occur inside the heart during arrhythmias such as ventricular fibrillation (VF) are complex and not fully understood. Through advancements in the field of cardiac mapping, researchers are able to better understand the complex electrical network of the heart and potentially decrease the occurrence of diseases like SCA $[2]$.

Electrical activity in the heart usually occurs in a well concerted sequence. During a VF event it is difficult to determine the exact time in which a particular area of tissue is activated because electrical activity in the heart no longer occurs in sequence and is chaotic.

It is important to know when tissue is being activated in order to deliver a successful defibrillation. The surface Laplacian electrogram is a measurement derived from multiple unipolar electrograms which provides accurate information on the time of local tissue activation [3].

Microelectrode arrays (MEA) are an enabling technology, which has been used in a wide variety of biological applications, including neural and cardiac signal recording and stimulation. There are several types of MEAs which have been developed for different applications. Two of the most often used types are the planar (flat) and the needle (three

dimensional) electrode. Several advantages and disadvantages exist between the two. For 
example, planar electrodes can be less difficult to fabricate than three dimensional needle electrodes and may be suitable for sensing or stimulation of relatively smooth tissues. However, needle electrodes can offer higher signal quality due to having greater electrode surface area, and are more suitable for sensing or stimulation of rougher tissue. The study outlined in this paper focuses on the use of a planar type MEA.

Current microelectrode array technologies suffer from issues such as poor signal to noise ratios (SNR) and/or spatial resolution [4]. Electrical noise limits the detail of information that arrays can provide by masking the signal of interest. This is especially true for biological potentials, where signal amplitudes are often very low (millivolts to microvolts) even with larger electrode sizes. As electrodes become smaller, noise increases dramatically [5]; a major issue since a key advantage of MEAs is the ability to resolve changes in electrical potential over progressively smaller distances, i.e. increase spatial resolution. To obtain accurate information on the cellular level, it is often necessary to decrease electrode size and spacing in order to obtain a high spatial resolution.

Previous work by our group [6] involved the design, development and characterization of a thin-film microelectrode array (TFMEA) directly interfaced to a custom signal conditioning microchip (SCM). This TFMEA-SCM was developed to acquire voltage potentials at the cellular level to calculate the surface Laplacian in cardiac tissue. Although the results from this initial device were promising, several limitations arose during the in vivo characterization of the device, such as reliability of the SCM when exposed to bodily fluids and difficulty obtaining low noise surface Laplacian signals. As a result, the team needed to redesign the TFMEA and signal conditioning component of the system in order to address these limitations. 


\subsection{Objective Statement}

The objective of this study was to modify the existing thin-film microelectrode arrays and signal conditioning components in order to improve the quality of recorded signals from the system to enhance the accuracy of the calculated surface Laplacian measurements obtained by the system.

\subsection{Hypotheses}

The SNR of the high spatial resolution recordings obtained with the modified TFMEA and signal conditioning board will significantly increase compared to the TFMEA-SCM system previously developed by our group. In order to prove the hypothesis the specific aims in section 1.4 will be achieved.

\subsection{Specific Aims}

Specific Aim 1: Decrease resistance of the metal trace from the electrode pad to bond pad by adding a thin layer of silver between the titanium and platinum layers. Noise will decrease with reduced electrical resistance, following the effects of Johnson-Nyquist noise.

Specific Aim 2: Develop an improved signal conditioning board to interface the MEA with the data acquisition device by using high performance off the shelf components. These devices feature ultra-low noise and input bias current and are application specific to devices such as the microelectrode arrays used in this project.

\subsection{Significance of the Study}

The results of in vivo testing from the previous TFMEA/SCM system demonstrated that the system could acquire cellular level potentials with good signal to noise ratios; 
however, the accuracy in the surface Laplacian calculations were highly dependent on the TFMEA placement location, i.e. epicardial vs. endocardial tissue. Having the ability to accurately acquire high spatial resolution data will enable the accurate determination of key cardiac parameters such as local activation time, transmembrane current density and the surface Laplacians of cardiac tissue. This information is critical to gaining a thorough understanding of the mechanisms responsible for cardiac arrhythmias. By improving the SNR, the accuracy of these key parameters should be improved leading to the development of better clinical therapies. 


\section{LITERATURE REVIEW}

\subsection{Electrocardiogram}

An electrocardiogram (ECG) is a summation of the electrical activity in the heart as seen on the surface of the body, most commonly taken in the thoracic region. An ECG provides a noninvasive method for measuring heart activity such as heart rate (pulse) and for identifying potential health risks associated with a diseased heart, such as arrhythmias. An idealized waveform of a normal ECG is shown in figure 2.1. The ECG signal is a measure of voltage amplitude versus time and is created from the difference in potentials obtained from several electrodes placed on the body's surface.

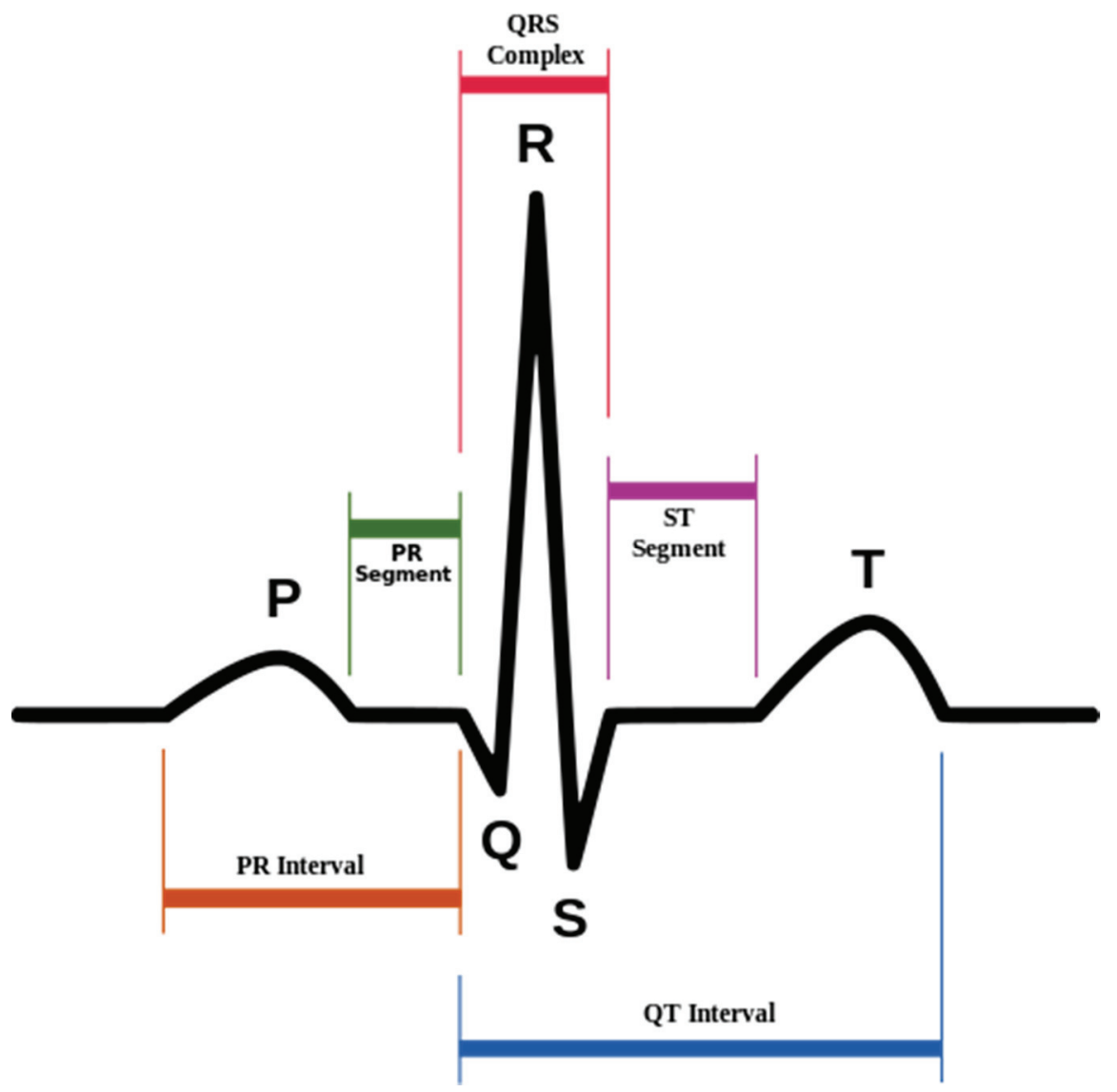

Figure 2.1 Idealized ECG waveform 
Each point and segment along the ECG waveform corresponds to a particular physical action in the heart. The $\mathrm{P}$ wave corresponds to atrial depolarization while the PR segment represents the delay of the action potential through the atrioventricular (AV) node. The QRS complex occurs during ventricular depolarization, which is also the period in which atrial repolarization occurs. The ST segment is the period during which the ventricles are contracting. Finally, the T wave represents ventricular repolarization and the end of a complete heartbeat.

\subsection{Arrhythmias}

Any deviation from normal electrical activity in the heart is known as an arrhythmia, of which there are many different types. For example, an abnormally fast heartbeat is called tachycardia and generally refers to a heart rate above 100 beats per minute. An abnormally slow heartbeat is called bradycardia and generally refers to a heart rate below 60 beats per minute. Other arrhythmias include those which cause an irregular heartbeat, such as atrial flutter, atrial or ventricular fibrillation, or heart block. Arrhythmias can be life-threatening to those affected because the normal flow of blood throughout the body is reduced or stopped in the case of ventricular fibrillation.

\subsection{MEA Cardiac Mapping Approaches}

\section{a. Work of others}

MEA technology has been successfully applied to both neurological and cardiac tissues in order to measure cellular voltage potentials as well as to stimulate these tissues. Kim et al [7] investigated the use of an MEA fabricated from iridium oxide $\left(\mathrm{IrO}_{\mathrm{x}}\right)$ coated film on silicon shanks for cardiac electrogram recording. A probe containing a single spike was applied to the left ventricle

of a mouse heart. The spontaneous and paced transmural potentials traversing between the 
epicardium to the endocardium were recorded. Additionally, a multiple spike electrode was used to record longitudinal cardiac wavefronts from a rabbit heart. Jacquir et al [8] designed an MEA contained within a substrate on which a monolayer of cardiac tissue was cultured. Excitation of action potentials was investigated using 60 electrodes spaced $200 \mu \mathrm{m}$ apart. This distance is considerably larger than the dimensions of a single cardiomyocyte and therefore limits the individual cell to cell potentials which can be detected. Frey et al [9] developed a CMOS compatible MEA with up to 126 selectable channels spaced $18 \mu \mathrm{m}$ apart. While this achieves sub-cellular level spatial accuracy, the device itself is large and not feasible for use during in vivo studies of cardiac tissue. Xin et al [10] developed a planar MEA to investigate sinoatrial (SA) node action potentials using a 2 by 50 electrode array. The array was fabricated using gold electrodes and SU-8 photoresist as a passivation layer. In addition, a custom ASIC was developed for this application. In addition to the physical construction of an electrode, its impedance plays a large role in the quality of recorded electrochemical signals. Reduction of this impedance is almost always beneficial. Layer et al [11] showed that the impedance of silver chloride, the type of electrode used in the work described in this paper, could be reduced by annealing it at $450{ }^{\circ} \mathrm{C}$ for two hours and then rapidly quenching it in cool air.

\section{b. University of Louisville TFMEA-SCM system}

Dong et al [6] developed and characterized a TFMEA-SCM system to investigate electrocardiograms, specifically surface Laplacian measurements, at the level of individual cardiomyocytes. The electrode array was fabricated using standard photolithographic techniques inside a class 100 cleanroom at the University of Louisville. Each array contained five groups of five electrodes. Two different sized arrays were designed onto the same photo mask. One of the two MEA designs had an effective area of $16 \mathrm{~mm}^{2}$ while the other had an effective area of $4 \mathrm{~mm}^{2}$. Within each group, the inter-electrode spacing was 
chosen to be $75 \mu \mathrm{m}$, which is the average size of a cardiomyocyte [12]. By choosing these dimensions it is possible to achieve accurate measurements at the cellular level, which can be considered high spatial resolution.
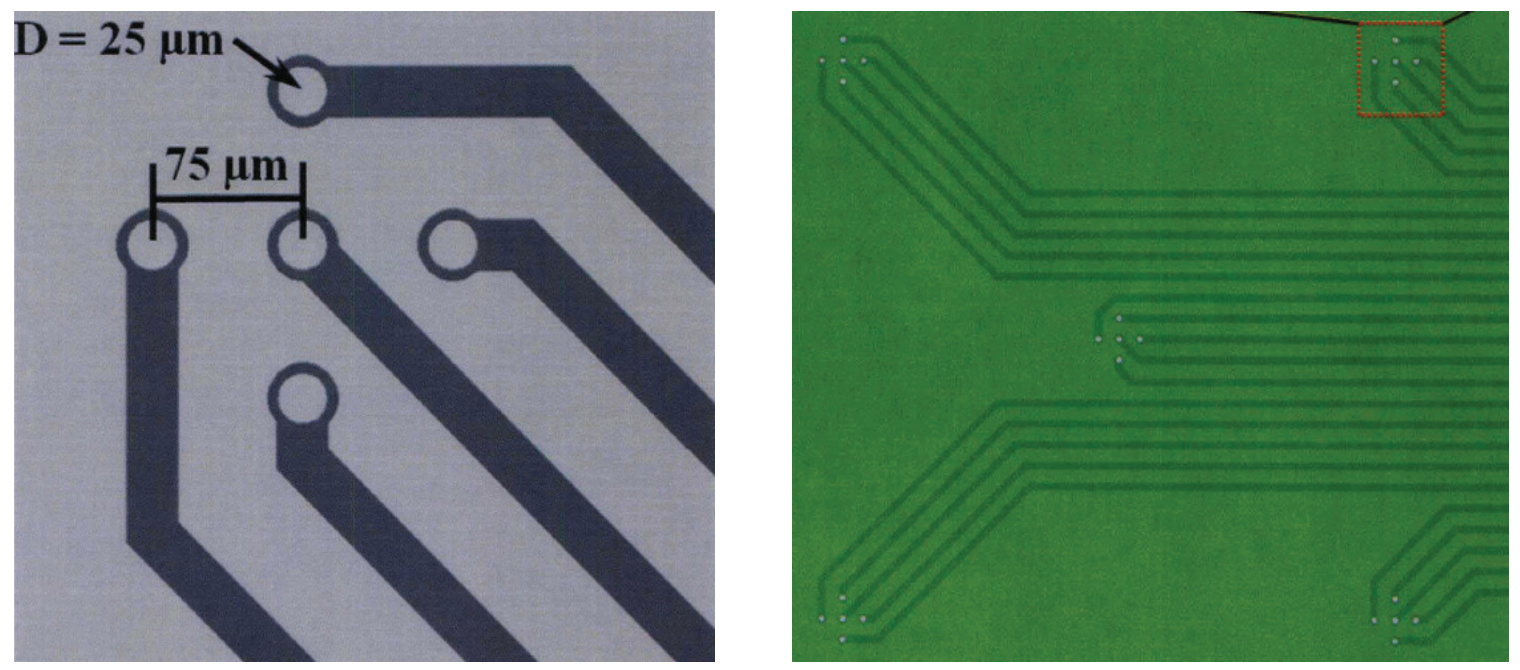

Figure 2.2 Left, inter-electrode spacing is shown to be $75 \mu \mathrm{m}$ in each group, diameter of each electrode is $25 \mu \mathrm{m}$. Right, five groups of five electrodes shown, total of 25 electrodes.

The results of in vivo testing proved that this TFMEA/SCM system could acquire cellular level potentials with high signal to noise ratio, approximately $40 \mathrm{~dB}$. Using this data acquisition system, biological properties such as local activation time and transmembrane current density of cardiac tissue were derived and studied. The ability to acquire such information makes this system an attractive option in the cardiac mapping field.

Some improvements for future development were suggested in this work. High spatial resolution measurements made on the order of $75 \mu \mathrm{m}$ did not produce better results in some cases, such as during endocardium surface Laplacian measurements. This was noted to possibly have been caused by the rough nature of the endocardium and could be 
improved if contact between the MEA and the tissue was improved, perhaps by forcing the array onto the tissue. Also, further miniaturization of the electrode array and/or signal conditioning system was suggested as a potential future improvement. Finally, wireless telemetry was suggested as a way to communicate with the data acquisition device, but some technical challenges such as data throughput given the high sampling rate and channel count were brought up as potential issues.

\subsection{Summary}

MEA technology has enabled researchers in fields such as cardiac mapping to investigate complex biological signals further than ever before. There is potential for expanding MEA use into the clinical setting in order to improve diagnostics and treatment. Microelectrode arrays provide great flexibility in that they can be used to sense or stimulate any electrogenic tissue. However, several challenges remain, such as ease and cost of fabrication, packaging methods, and signal quality versus electrode density. 


\section{INSTRUMENTATION AND EQUIPMENT}

\subsection{Fabrication}

Fabrication of the TFMEA devices was performed in the University of Louisville micro/nano technology center (MNTC) class 100 cleanroom. The MNTC cleanroom is a full featured facility that provides access to highly specialized equipment and the environment needed to fabricate MEMS devices and integrated circuits. This section describes the materials and equipment that were used in the MNTC cleanroom during fabrication of the TFMEA.

Headway spinners (Headway Research, Garland, TX) were used to spin coat HD Microsystems (Hitachi/DuPont Chemical) polyimide PI2611 onto the oxidized silicon wafer as a bottom and top layer for the TFMEA body. HD Microsystems photoresist 1827 was spin coated on top of the polyimide layer after curing and used to apply photomask number one. After metal deposition and coating of top polyimide layer, HD Microsystems photoresist 1813 was spin coated on top of the polyimide layer and used to apply photomask number two and number three.

Yield Engineering Systems (Livermore, CA) vacuum oven (YES-550PB6/81122P-CP) shown in figure 3.1 was used after coating the silicon wafer with polyimide to fully cure it. This oven can be programmed with different profiles in order to ramp up, hold, and ramp down to specified temperatures over a given period of time. The atmosphere inside the chamber is also controllable. Various different gases which may be connected to the oven can be selected and their pressures inside the chamber specified over the duration of curing. 


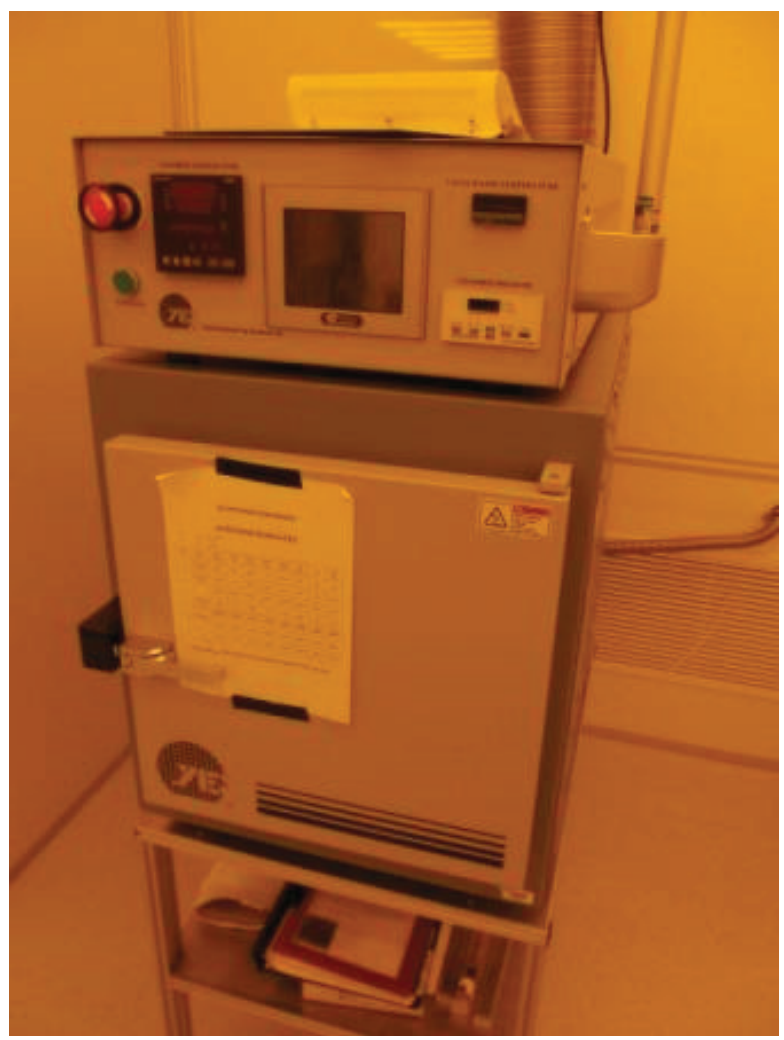

Figure 3.1 YES Vacuum Oven

After the polyimide was cured, the SUSS MicroTech aligner shown in figure 3.2 was used to apply photomask number one onto the substrate which had been coated with a photoresist. Necessary exposure times increased as the light intensity of the mercury lamp used to activate the photoresist decreased. A log was kept of each exposure by each user, so it was possible to estimate the necessary exposure time for each use of the aligner. On average, this was around 30 seconds for photoresist 1827 and 15 seconds for photoresist 1813. 


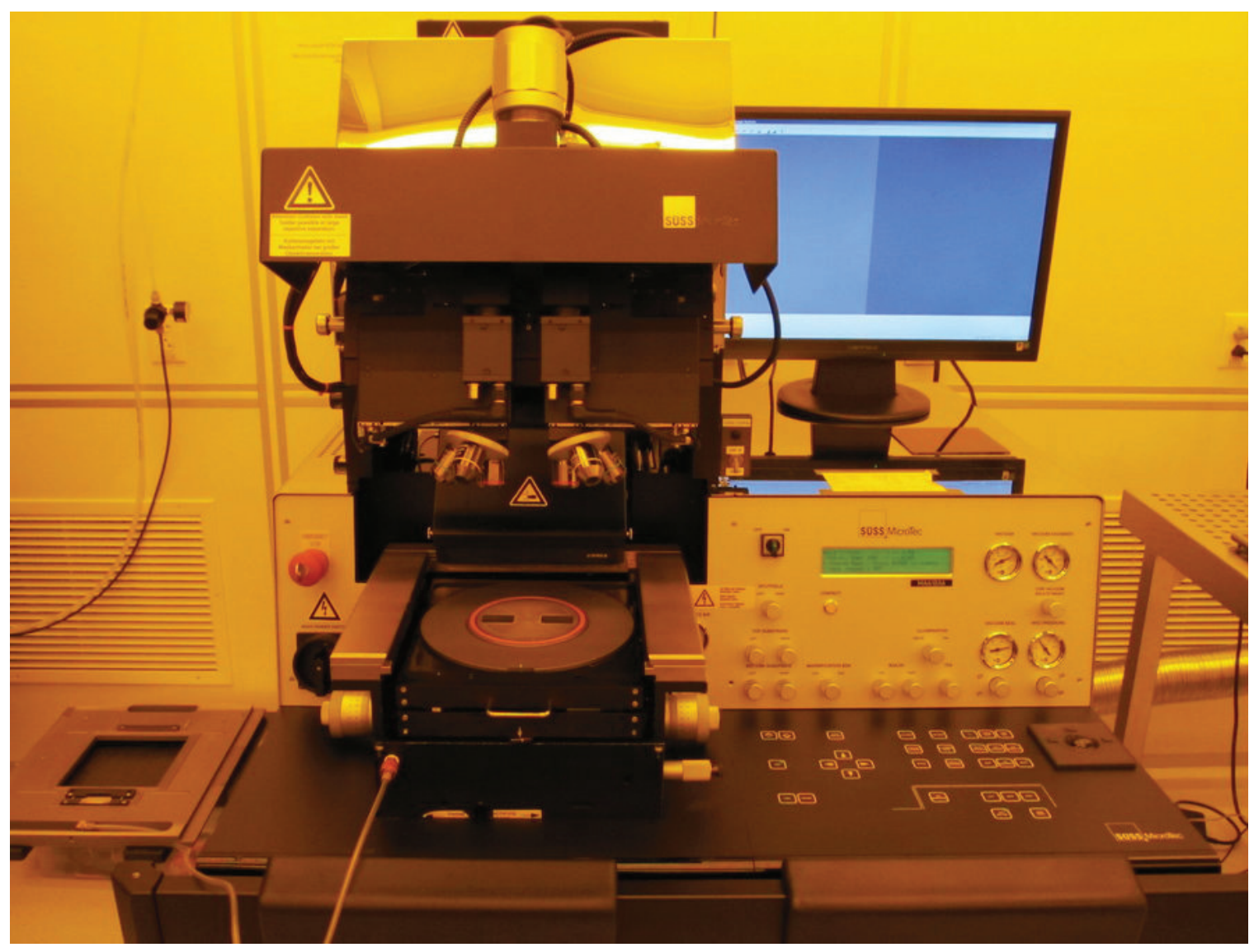

Figure 3.2 SUSS MicroTech mask aligner

Yield Engineering Systems (Livermore, CA) image reversal oven (310-TA) shown in figure 3.3 was used along with $\mathrm{NH}_{3}$ gas after application of mask one to reverse the polarity of the photoresist. Mask one was designed as an inverse of the actual pattern needed. This was done because it increases the resolution of the mask pattern as it is transferred to the photoresist. There was a fifteen minute wafer warm up delay, after which the ammonia gas flowed into the chamber. The gas was located a considerable distance away from the oven and this caused an issue once the gas began to flow. It was necessary to enter the storage area to reset the alarm several times until the gas was primed. 


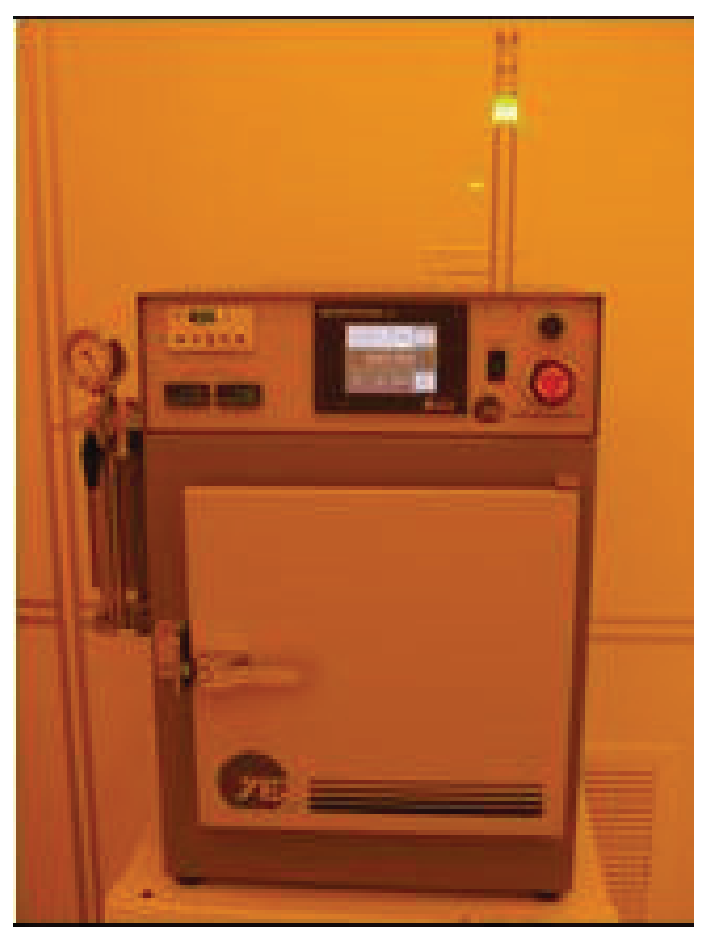

Figure 3.3 YES Image Reversal Oven

Kurt J Lesker PVD75 (Jefferson Hills, PA) sputtering system shown in figure 3.4 was used with several metal targets to create the metal traces which run from the bond pads to the electrode pads. The PVD75 allows three separate metal targets to be used in one run. Two 3" sputtering guns and one 4" gun are available. DC up to $1500 \mathrm{~W}$ and RF up to 300W sputtering options are present, however only DC sputtering was used. A titanium 3" target was used as the first metal layer to provide excellent adhesion of the subsequent metal layers to the polyimide. A silver 4" target was used as the second metal layer in order to reduce electrode trace resistance. The final trace metal was platinum and a 3" target was used to provide a surface for silver electroplating. A 4" aluminum target was needed to act as an etch stop layer when opening the electrode vias. A Technics sputtering system was used with the aluminum target for troubleshooting aluminum sputtering issues. 


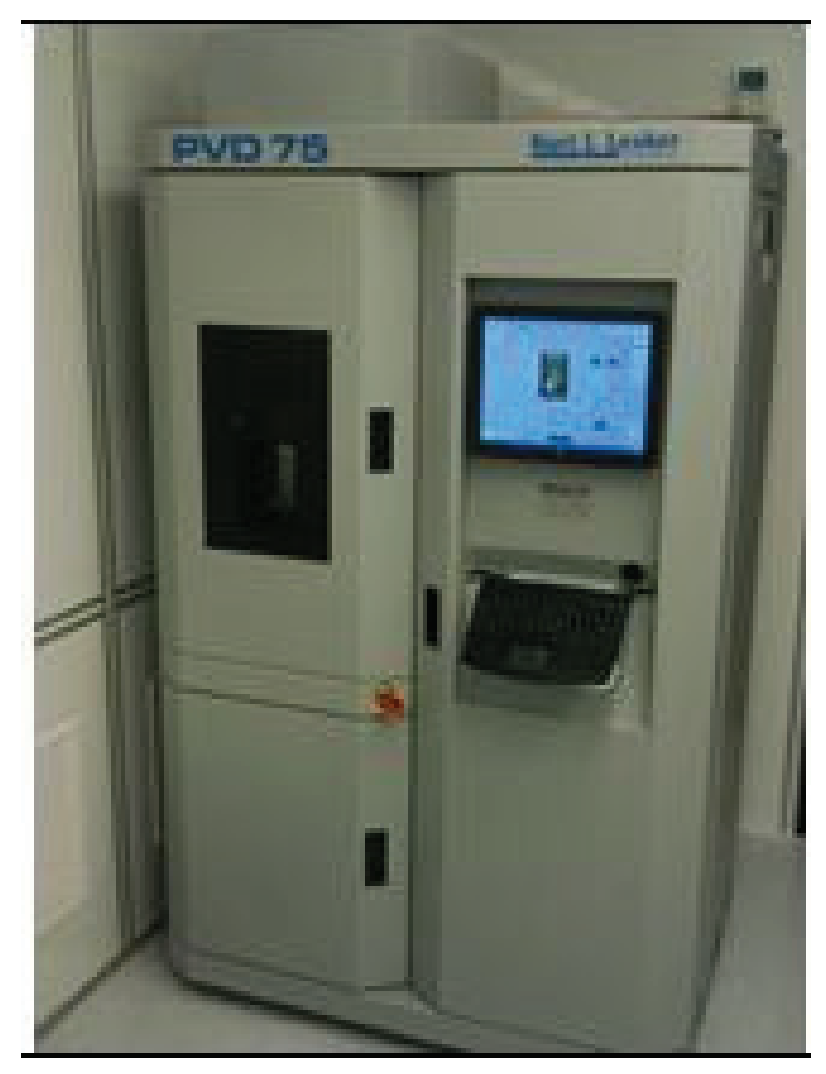

Figure 3.4 Kurt J Lesker PVD75 sputtering system

Nordson March CS-1701 reactive ion etching (RIE) machine (Concord, CA) shown in figure 3.5 was used to etch anisotropic vias in the top polyimide layer in order to open the electrode contacts below and also to roughen the polyimide surface in preparation for sputtering. The RIE was used with oxygen and $\mathrm{CF}_{4}$ gases to perform etching. Oxygen gas forms a plasma inside the chamber which etches exposed polyimide. Tetrafluoromethane gas helps to clean up the ash that is produced from etching the polyimide, so that the electrode pad is clean and suitable to be electroplated. 


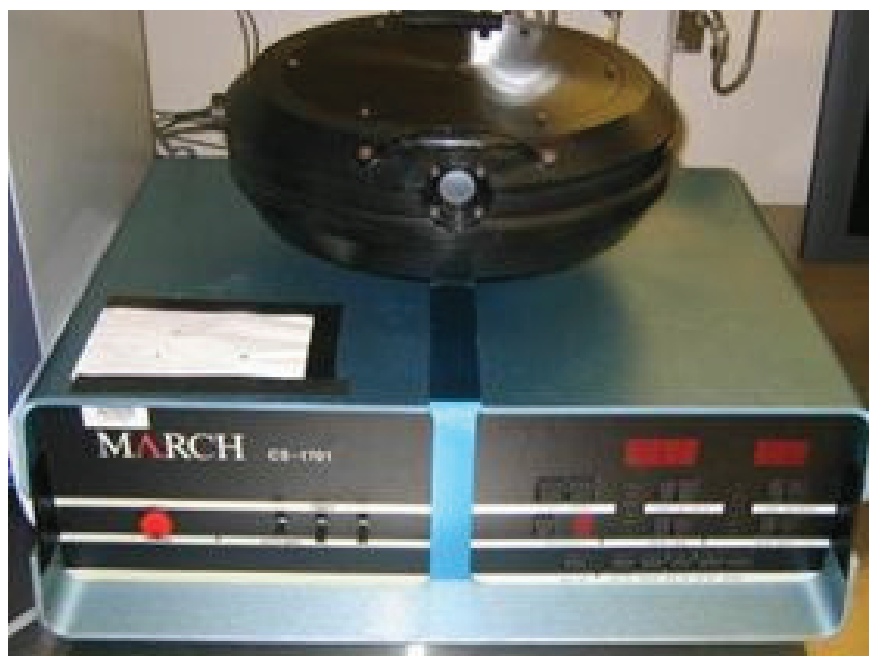

Figure 3.5 March reactive ion etcher (RIE)

Veeco Dektak 8 profilometer (Plainview, NY), shown in figure 3.6, was used after reactive ion etching to measure the depth of the vias leading to the electrode's platinum layer beneath. This was very important to ensure that the platinum had been exposed and that no polyimide was left in the via, which would cause failure of the silver electroplating step. Several times during the silver electroplating step the Dektak 8 was used to determine when the via had been fully plated with silver.

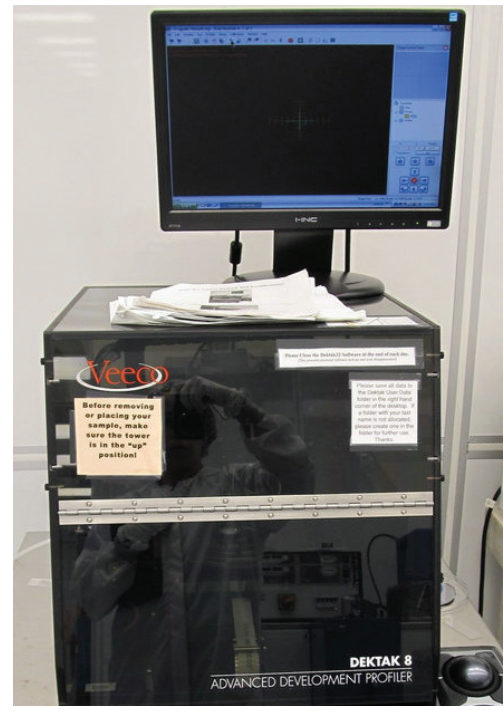

Figure 3.6 Veeco Dektak 8 Profilometer 
Technic silver cyless II was premixed by Technic (Cranston, RI) and used to electroplate silver in the via of each electrode up to the surface of the top polyimide layer. This product did not contain cyanide, which is commonly found in electroplating solutions. Silver cyless II has a stated shelf life of six months. A Dynatronix DuPR 10-3-6 pulse plating supply provided the correct pulse waveform to electroplate the silver. An Agilent $34410 \mathrm{~A}$ precision current meter was needed to measure on the microampere scale to determine correct electroplating current.

\subsection{Test and Packaging}

Once the TFMEA had been fabricated it was necessary to ensure that each sensor was fully functional before it could be used for in vivo studies. TX-151 powder (Oil Center Research, Lafayette, LA) was used along with agar and sucrose to create an electrically conductive gel on which the TFMEA could be tested. Sucrose was mixed with deionized water and heated to boiling point, agar was then added and mixed well. Upon addition of the pink TX-151 powder the mixture thickened and was poured into plastic containers to cool. Metal electrodes were placed on either end to enable a voltage potential to be applied across the gel, simulating cardiac tissue.

A National Instruments USB-6353, 32 channel X-series data acquisition (DAQ)

device was used to verify MEAs and signal conditioning boards in the lab before in vivo data collection. This DAQ has 32-channels of high speed, 16-bit analog to digital converters suitable for acquiring all 25 channels on the TFMEA. National Instruments LabVIEW 2011 was used to collect data and perform data filtering and analysis. 


\subsection{Signal conditioning board design and testing}

The design and simulation of the new signal conditioning board was performed mainly

using National Instruments circuit design software, Multisim. This CAD software allows a schematic to be laid out using resistors, capacitors, and opamps and then simulated. Multisim was very useful in prototyping the circuit that was used for amplification and band-pass filtering of the TFMEA signals.

ExpressPCB CAD software was used to create the printed circuit board layout of the new signal conditioning board. This is a software package which is distributed with the ExpressPCB manufacturing service and allows a user to design their two or four layer circuit board to be manufactured. Once the completed design was submitted, ExpressPCB manufactured and shipped the completed boards. One percent tolerance resistors and capacitors as well as the operational amplifiers from Texas Instruments were purchased from Digikey (Thief River Falls, MN) and used to populate the new circuit boards. 


\section{PROCEDURE}

A TFMEA and SCM system for cardiac extracellular potential measurements was previously designed and characterized [6]. This section focuses on the procedures followed to further improve and develop these devices. The first area of improvement targeted was to decrease the electrical resistance of the thin metal traces in order to improve signal transmission and reduce noise. The second area focused on designing a new SCB system to interface the TFMEA to data acquisition hardware.

\subsection{Brief Overview of Previous Generation System}

This thesis was based on the work of Dong et al, in which a complete TFMEASCM system was developed and characterized. This work proved to add valuable information to the field of cardiac mapping by allowing researchers to see cardiac electrical activity at the cellular level. The SCM was realized as a custom, high channel count, integrated circuit conditioning system which directly interfaced with the TFMEA to increase SNR.

The TFMEA fabrication methods employed in the prior work were followed, with the addition of silver to reduce electrode trace resistance. The SCM device was at first used, but issues with its performance led to the creation of an all new signal conditioning board (SCB) for this thesis. This new SCB employed the use of high quality off the shelf operational amplifiers, and provided the same functionality as the SCM, but with increased performance. These improvements included lower noise, lower input bias current, lower input offset voltage, as well as being application specific to sensors and having much more robust electrostatic discharge protection. 


\subsection{Design improvements}

Each of the 25 metal traces was designed to be $25 \mu \mathrm{m}$ thick; this parameter was not modified. On average each metal trace was $36 \mathrm{~mm}$ in length, the distance from the bonding pads to the electrode itself. This parameter was also not modified so that the MEA retained its previous size and shape. In order to decrease electrical resistance, the metal layers were modified to include a third metal layer comprised of silver $(\mathrm{t}=200 \mathrm{~nm})$ sandwiched between the previous design that consisted of two metal layers, titanium $(\mathrm{t}=20 \mathrm{~nm})$ and platinum $(\mathrm{t}=200 \mathrm{~nm})$. Theoretical calculations of the expected electrical resistance in the metal traces for previous generation and new generation TFMEAs are shown below.

Equation for calculating the resistance of a material:

$$
R=\frac{\rho L}{A}
$$

Length of each electrical trace, on average:

$$
L \approx 36 \mathrm{~mm}
$$

Cross-sectional area of metals on each trace:

$$
\begin{gathered}
A_{T i}=25 \mathrm{um} * 20 \mathrm{~nm} \\
A_{A g}=25 \mathrm{um} * 200 \mathrm{~nm} \\
A_{P t}=25 \mathrm{um} * 200 \mathrm{~nm}
\end{gathered}
$$

Resistivity constants of metals used:

$$
\begin{gathered}
\rho_{T i}=4.2 * 10^{-7} \Omega m \\
\rho_{A g}=1.59 * 10^{-8} \Omega m \\
\rho_{P t}=1.06 * 10^{-7} \Omega m
\end{gathered}
$$

Theoretical resistance of a single trace, previous TFMEA generation:

$$
R_{\text {old }}=R_{T i} \| R_{P t}=763.2 \Omega
$$

Theoretical resistance of a single trace, new TFMEA generation:

$$
R_{\text {new }}=R_{T i}|| R_{A g} \| R_{P t}=99.5 \Omega
$$


A decrease in electrical resistance will always cause a reduction in electrical noise. This is due to the effects of Johnson-Nyquist noise, also known as thermal noise. The equation for this noise is shown:

$$
v_{n}=4 k_{B} T R
$$

This equation states that the noise voltage is proportional to temperature and resistance. As either temperature or resistance is reduced noise is also reduced.

Note that reducing the trace resistance from $\sim 1000 \Omega$ to $\sim 100 \Omega$ may have little effect on the input voltage to the amplifier, since the input resistance is so large. However, an input current must flow to bias and turn on the FET gates of the operational amplifier and this current flows from the electrode through the metal trace itself. Thus, any reduction in resistance is beneficial, especially if low quality amplifiers are used in which large bias currents are required for the amplifiers to function. Another issue that exists is the mutual capacitance and inductance between the 25 metal traces. Since the traces are spaced so close together, when the resistance is high, the effects of capacitive or inductive coupling between neighboring traces could potentially become an issue. This issue is unlikely a major concern in the low frequency range of electrocardiograms $(0.5 \mathrm{~Hz}$ to $1 \mathrm{kHz})$, but should be minimized to the extent feasible nonetheless.

In addition to directly reducing the resistance of the electrical traces with the addition of silver, some wafers with finished TFMEAs were annealed at $450{ }^{\circ} \mathrm{C}$ under argon for two hours [11] to further reduce the impedance of the electrodes. Additionally, the annealing was performed to increase the adhesion of the silver chloride layer to the electroplated layer, since the silver chloride layer had a propensity for shearing off or having low durability. However, a concern with this process was that the glass transition 
temperature of polyimide PI2611 is $360{ }^{\circ} \mathrm{C}$, which may lead to flow of the polyimide and alteration in the structural features of the TFMEA. As a result, electrode height measurements were taken before and after annealing for comparison using the Dektak 8 profilometer described in Chapter 3.

\subsection{Fabrication}

Starting with a new four-inch silicon oxide $(500 \mathrm{~nm})$ wafer, polyimide PI2611 from HD Microsystems was spun on to the wafer using a spinner from Headway Research Inc. Table 4.1 shows the spin parameters for the first coating of polyimide. Once the wafer was centered and vacuum seated to the chuck, a quarter sized amount of PI2611 was poured on the wafer. After the spinner was activated the wafer was accelerated to $500 \mathrm{rpm}$ and held there for 5 seconds. After 5 seconds the wafer was accelerated to $4000 \mathrm{rpm}$ and held there for 30 seconds. A clean piece of paper was folded and held in close proximity to the wafer as it spun in order to prevent residue from migrating underneath the substrate. If residue ended up on the bottom of the substrate, subsequent mask alignment steps were difficult to perform because hard contact of the mask to the substrate was not achievable. The cured polyimide thickness at the final spin speed of $4000 \mathrm{rpm}$ was approximately $4.5 \mu \mathrm{m}$.

\section{Table 4.1 Polyimide spin parameters}

\begin{tabular}{|l|c|c|c|}
\hline & Spread & Spin & Stop \\
\hline Speed (rpm) & 500 & 4000 & 0 \\
\hline Time (s) & 5 & 30 & - \\
\hline
\end{tabular}

The coated wafer was allowed to sit for one minute on the vacuum chuck to allow the edges to level off where polyimide has a tendency to accumulate. This also helped to reduce imperfections in the polyimide, such as bubbles or streaks that may have formed 
during spinning. The wafer was soft baked on a hotplate at $115{ }^{\circ} \mathrm{C}$ for two minutes to evaporate solvents and allow the polyimide to be cured.

After removing from the hotplate the wafer was placed in a glass wafer boat holder and placed in the Yield Engineering Systems (YES) polymer curing oven. Program number four was used to cure the polyimide. The temperature profile for this program is shown in Table 4.2. The idle temperature of the oven is held at $50{ }^{\circ} \mathrm{C}$ and ramps up to $350{ }^{\circ} \mathrm{C}$ over three hours. Once the oven reaches $350{ }^{\circ} \mathrm{C}$ it holds this temperature for one hour. After this the oven decreases the temperature back to $50{ }^{\circ} \mathrm{C}$ over three hours and then curing is complete.

Table 4.2 Polyimide curing parameters

\begin{tabular}{|l|c|c|c|}
\hline Step & 1 & 2 & 3 \\
\hline Temperature $\left({ }^{\circ} \mathrm{C}\right)$ & 50 & 350 & 50 \\
\hline Ramp rate $\left({ }^{\circ} \mathrm{C} / \mathrm{hr}\right)$ & 100 & 0 & -100 \\
\hline Time (hrs) & 3 & 1 & 3 \\
\hline
\end{tabular}

After curing, the wafer was briefly cleaned with acetone and DI water and fully dried to remove any contaminates that may have existed in the oven. Photoresist 1827 was spin coated on the wafer with a 0.2 second spread at $500 \mathrm{rpm}$ with a final speed of 4000 $\mathrm{rpm}$ for ten seconds and then soft baked at $115{ }^{\circ} \mathrm{C}$ for one minute. Using the SUSS MA6 mask aligner, photo mask number one shown in Figure 4.1 was applied to the wafer using an ultraviolet exposure time of approximately 25 seconds. 


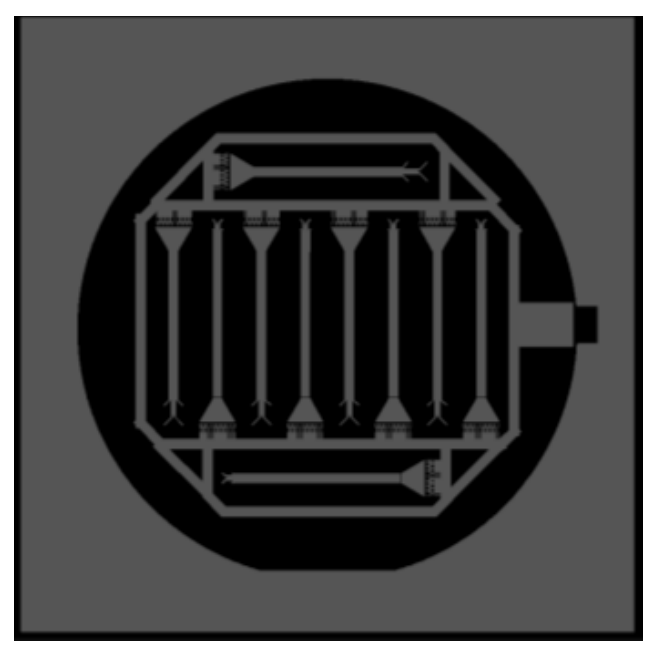

Figure 4.1 Photomask number one

Mask number one provided an inverted pattern for the metal traces of the electrode arrays. Before the metal traces could be sputtered, reversal of the transferred mask was necessary. By the use of an inverted mask followed by image reversal, the correct geometries for the metal traces were created. By using an inverted mask it was possible to use photoresist 1827, which is a positive photoresist.

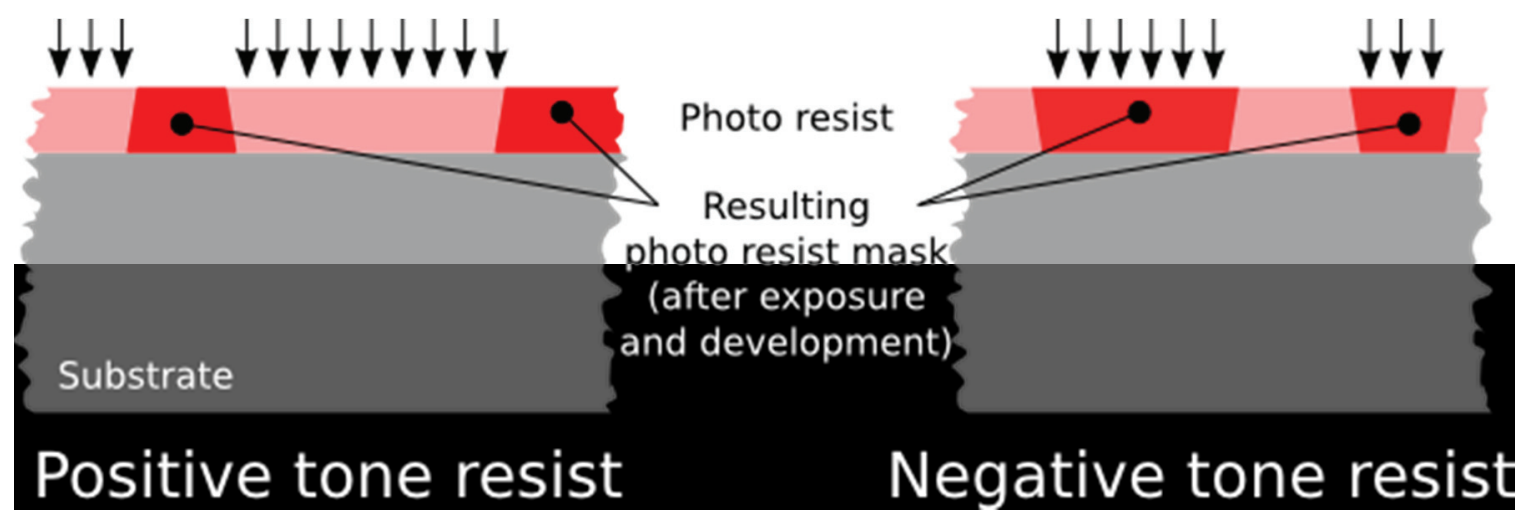

Figure 4.2 Positive versus negative photoresist [13]

By using a positive photoresist it was possible to achieve higher resolution on the sputtered metal traces and electrode pads. See Figure 4.2 for a visual representation, notice that the 
top of the photoresist has a smaller aperture than that of the negative photoresist. Image reversal was performed at $90{ }^{\circ} \mathrm{C}$ in the YES 310-TA image reversal oven under $\mathrm{NH}_{3}$ gas for 45 minutes. There was a fifteen-minute wafer warm up delay, after which the user needed to enter the maintenance area of the cleanroom to reset the ammonia alarm as the oven begins to perform the image reversal. The image reversal step renders the exposed photoresist insoluble by the developer.

Once image reversal was complete the wafer was flood exposed using the SUSS MA6 aligner for 30 seconds in ultraviolet light without a mask, causing only the previously un-exposed areas to become activated. Next, the wafer was developed using Rohm and Haas Microposit MF-319 developer for one and a half minutes while gently agitated, using a medicine dropper to repeatedly pass developer across the wafer. The wafer was washed with DI water and dried. To ensure that all photoresist had been removed from the metal trace patterned areas, a cotton swab was wet with acetone and gently brushed on the surface in a large area where resist should have been completely removed. If any resist was still present, a streak would appear and further development was required in 30 second increments. During fabrication of the first few improved TFMEAs an artifact, shown in Figure 4.3, was noticed after development. 

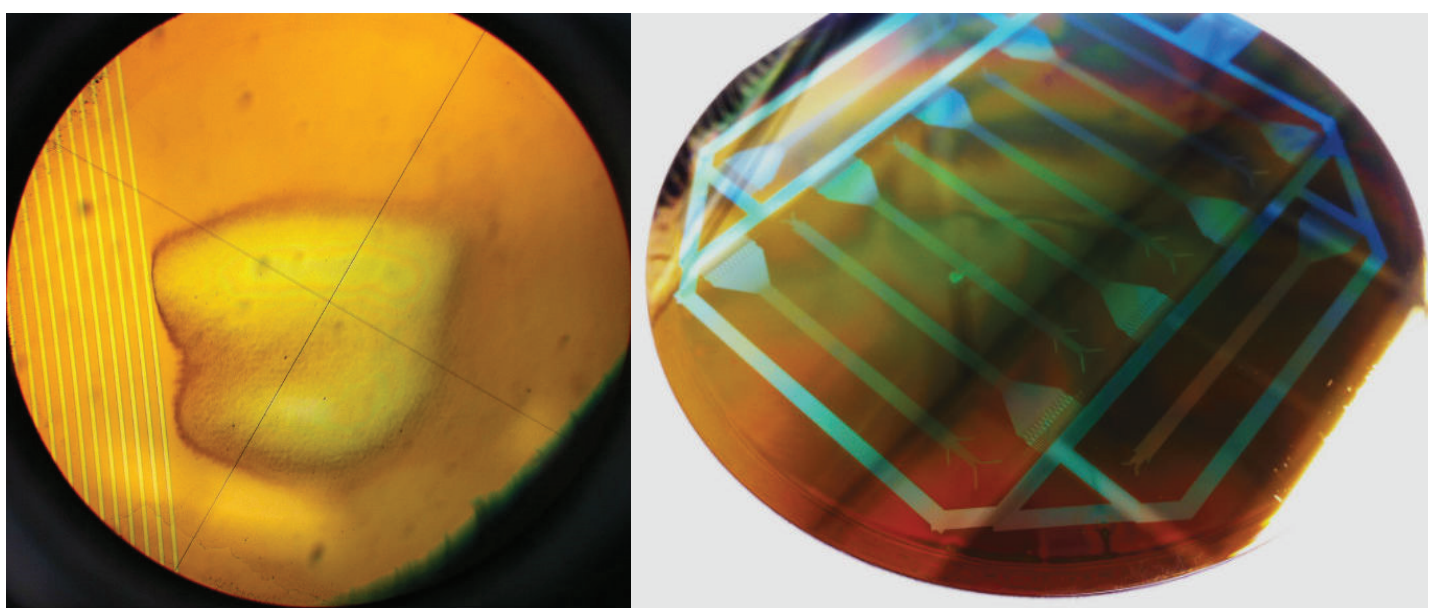

Figure 4.3 Artifact found during development of mask number one

This artifact was repeatedly found on other wafers during fabrication. The cleanroom staff was notified and it was found that some type of photoresist had been deposited on the exposure lamp, causing light to be scattered in that area which caused the artifact to appear on a fully developed wafer.

Before metal traces were sputtered onto the patterned area, the surface of the wafer was roughened using the March RIE (reactive ion etching) for ten seconds at $300 \mathrm{~W}$, under an oxygen pressure of 250 mTorr with $20 \mathrm{sccm}$ flow rate. This step removed any traces of photoresist that could have still been in the pattern areas and allowed for better adhesion of the metals during lift-off and in subsequent processing steps.

Sputtering was performed on top of the patterned photoresist and the polyimide below it to create the metal traces. The Kurt J. Lesker PVD 75 machine has the ability to use three metal targets, two different three inch targets and one four inch target. The first metal to be deposited was titanium, using a three-inch target. Through characterization, it was found that two minutes of sputtering at $300 \mathrm{~W}$ under 5 mTorr argon pressure yielded a film thickness of $20 \mathrm{~nm}$. This thin layer of titanium provided excellent initial adhesion to the polyimide and acted as an interface with the metal layers above it. In order to reduce 
the resistance of the thin metal traces, the next metal deposited was silver, using a four inch target. $300 \mathrm{~W}$ of power under 5 mTorr argon pressure for three minutes and ten seconds was required to yield $200 \mathrm{~nm}$ of silver, which was the target thickness calculated for decreasing the resistance of the $25 \mu \mathrm{m}$ thick metal traces by a factor of 10 . Finally, platinum was sputtered using a three inch target. It was found that five minutes of sputtering at 300 W under 5 mTorr argon pressure yielded a $200 \mathrm{~nm}$ layer platinum. Pre-sputtering of these metal targets in the PVD75 was critical to achieve a good initial deposition, especially in the case of titanium. Approximately 30 seconds of sputtering with the source and substrate shutters closed was enough. Between sputtering each metal, two minutes of cool down was allowed to prevent thermal damage to the polyimide and photoresist.

Once deposition of the metal traces was completed, the excess metal on top of the photoresist was removed by placing the wafer in acetone for a period of several hours to dissolve the photoresist. A medicine dropper filled with acetone was then used to target individual areas of the wafer until all excess metal was removed. The wafer was cleaned with fresh acetone, rinsed well with DI water and finally dried with the nitrogen gun. The wafer was placed on the $115{ }^{\circ} \mathrm{C}$ hotplate for ten to twenty minutes to evaporate any residual acetone that may have absorbed into the polyimide. It was found that if this step was not performed, bubbling occurred when the second layer of polyimide was applied and cured, which rendered the wafer useless.

Another layer of polyimide PI2611 was spun onto the newly patterned metal traces using the same parameters as indicated above in Table 4.1, except at a final spin speed of $5500 \mathrm{rpm}$ was used that resulted in a final cured thickness of approximately $3 \mu \mathrm{m}$. The higher final spin speed produced a thinner top layer and made the etching process easier 
when opening the electrode vias for the electroplating step. The newly coated wafer was soft baked at $115^{\circ} \mathrm{C}$ for two minutes and cured again at the same settings as before in the same oven. After curing, reactive ion etching was once again performed for ten seconds at $300 \mathrm{~W}$ under $250 \mathrm{mTorr}$ of oxygen pressure at $20 \mathrm{sccm}$ flow rate to roughen up the polyimide surface and allow for better adhesion of the aluminum layer during the next process.

Aluminum was sputtered onto the second polyimide layer surface as an etch stop layer, with a target thickness of $200 \mathrm{~nm}$. After deposition of aluminum, photoresist 1813 was spun on top of the aluminum with an initial spread spin at $500 \mathrm{rpm}$ for 1 second with a final spin speed of $4000 \mathrm{rpm}$ for 10 seconds. The wafer was then soft baked for one minute at $115^{\circ} \mathrm{C}$. Using the SUSS MA6 mask aligner, mask number two shown in Figure 4.4 was applied and exposed for approximately 15 seconds.

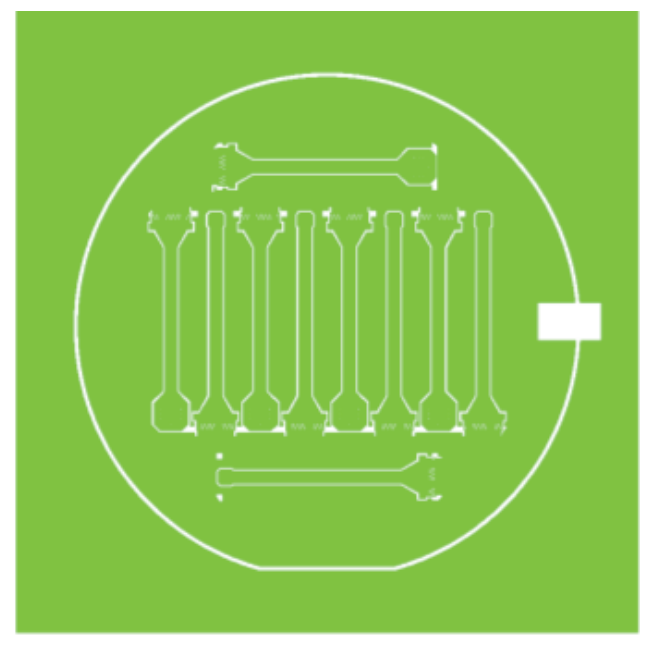

Figure 4.4 Photomask number two

Alignment of this mask was the most critical step since it determined the location where the microelectrode openings (holes) would be etched (through to the platinum layer) relative to the metal traces. Alignment markers existed below the second polyimide layer on the substrate and care was taken to align them well with the second mark. The exposed 
wafer was then developed in MF-319 developer. After development, the wafer was hard baked for 20 minutes at $115{ }^{\circ} \mathrm{C}$ to ensure stability of the photoresist during the subsequent aluminum wet etching step. After the hard bake, the wafer was wetted with DI water and placed in an aluminum etchant for approximately two minutes until the outline of the second photo mask was clearly visible. Following wet etching, the wafer was dried and inspected under the microscope to ensure complete etching of the aluminum.

A special note on aluminum sputtering: When using the KJL PVD75 to sputter the aluminum etch stop, some interesting characteristics were noted. A four-inch aluminum target had been freshly sand blasted and cleaned to remove previous contamination. When using the aluminum target at $300 \mathrm{~W}$ under 5 mTorr argon pressure, plasma was visible as a violet glow indicating that deposition was occurring. The sputtering voltage was around $225 \mathrm{~V}$ and the current around 1.3 A. However, after several minutes of sputtering no deposition was visible or measureable using the Dektak 8 profilometer. It was found that by initially increasing the argon pressure to a very high value, $\geq 50 \mathrm{mTorr}$, then performing a pre-sputtering step with the aluminum and gradually bringing the pressure back to 5 mTorr caused activation of deposition. The sputtering voltage after the high argon pressure exposure increased to $330 \mathrm{~V}$, the current decreased to around $0.9 \mathrm{~A}$ and a good deposition was achieved.

After aluminum wet etching, the substrate was placed in the March RIE at a power of $300 \mathrm{~W}$ under $250 \mathrm{mTorr}$ of oxygen pressure at $20 \mathrm{sccm}$ flow rate for four minutes. The RIE was powered off for two minutes to allow the substrate time to cool down slightly, 
then the power was turned on again for four additional minutes. Subsequently, the wafer was inspected visually and under an optical microscope. It is desired to have no polyimide remaining in the $25 \mu \mathrm{m}$ microelectrode openings (holes) for the platinum plating process. In some instances, a polyimide residue was seen by slowly moving the microscope objective up and down and observing the edges of the electrode holes to see whether concentric rings appeared via refracted light. If residue was present, additional reactive ion etching processes were performed at two minute intervals to completely etch through the polyimide. Care was taken not to over etch because over-etching tended to open the microelectrode openings (holes) more than the designed $25 \mu \mathrm{m}$, which directly affected the electroplating process characteristics and the calculation of the surface Laplacian, which is dependent on inter-electrode spacing.

Once complete dry etching had been confirmed, the wafer was placed in an aluminum wet etch again to completely remove all of the aluminum, which generally took 20 minutes. After the final wet etching, the wafer was cleaned using the ultrasonic cleaner, in water, at $60{ }^{\circ} \mathrm{C}$ on power level two for ten minutes to remove any nano-sized particles that may have adhered to the electrode pads, such as ash from the photoresist or polyimide. The wafer was once again washed with DI water, dried, and inspected under the microscope. Any additional ultrasonic cleaning was performed until the electrode pads were completely clean.

Photoresist 1813 was once again spun on the wafer, with electrode holes now open and ready for silver electroplating. A third and final photo mask, shown in Figure 4.5, was applied for approximately 15 seconds. 


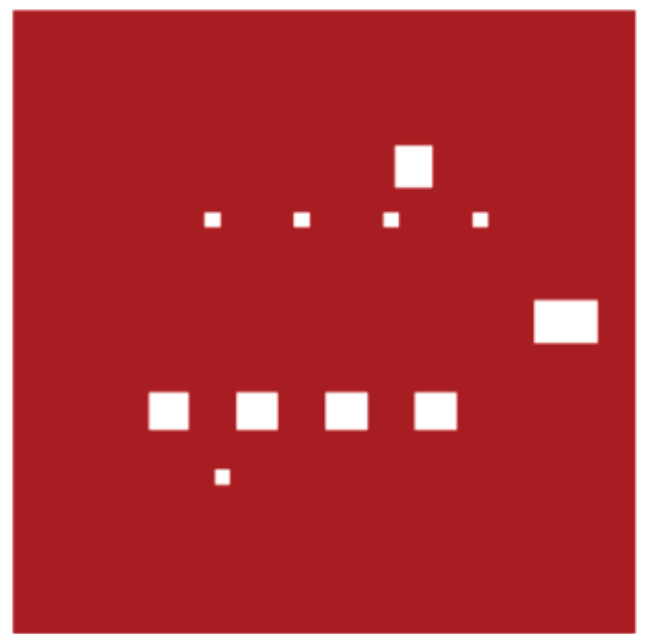

Figure 4.5 Photomask number three

This mask covered the entire wafer except where the silver electroplating connection pad and the electrodes were located. The wafer was developed in MF-319 developer and then re-exposed for a second time and re-developed to ensure that no photoresist was left in the electrode holes. The wafer was hard baked for 20 minutes to ensure stability in the electroplating solution and reduce excess current leakage from areas not plated. Finally, a ten second reactive ion etch was performed at $300 \mathrm{~W}$ under 250 mTorr of oxygen pressure at $20 \mathrm{sccm}$ flow rate to once again ensure the electrode pads were completely clean.

In the final processing steps of the thin film microelectrode array, the freshly exposed platinum electrode pads were electolytically-plated with silver up to several hundred nanometers above the second polyimide surface. Silver electroplating proved to be one of the most challenging parts of the fabrication process. Typically for MEMS applications, an acceptable plating current density should be in the $2 \mathrm{~mA}$ to $20 \mathrm{~mA}$ per square $\mathrm{cm}$ range $[6,14]$. The current density value can be used to calculate the plating current needed to plate the 250 electrode pads per wafer:

Radius of one electrode: 


$$
r=12.5 \times 10^{-6} \mathrm{~m}
$$

Total area to be plated:

$$
A_{\text {total }}=250 \times \pi \times r^{2}=0.0012 \mathrm{~cm}^{2}
$$

Given current density:

$$
I_{d}=\frac{20 m A}{c m^{2}}
$$

Total current to be used for plating, on average:

$$
I_{\text {total }}=I_{d} * A_{\text {total }}=24 \mu A
$$

According to the calculations above, an average plating current of $24 \mu \mathrm{A}$ should be maintained. Electroplating was performed using a Dynatronix DuPR 10-3-6 pulse plating power supply. The silver electroplating solution used was Technic Silver Cyless II, which is a cyanide free silver plating solution. A $100 \mathrm{~ms}, 40 \%$ duty cycle pulsed DC waveform was used for plating. An Agilent oscilloscope, model DSO1004A, was connected in parallel with the output of the plating power supply and an Agilent model 34410A precision current meter was placed in series between the plating power supply and the pure silver anode in order to measure the average plating current.

Silver Cyless II solution was added to a clean one-liter beaker placed on a hot plate, with a magnetic stir bar on the bottom of the beaker. The hot plate stirrer was turned on to provide gentle agitation of the solution. The wafer to be plated, or cathode, and the pure silver anode were placed parallel to each other and held in place with two wafer holders mounted together, see Figure 4.6. 


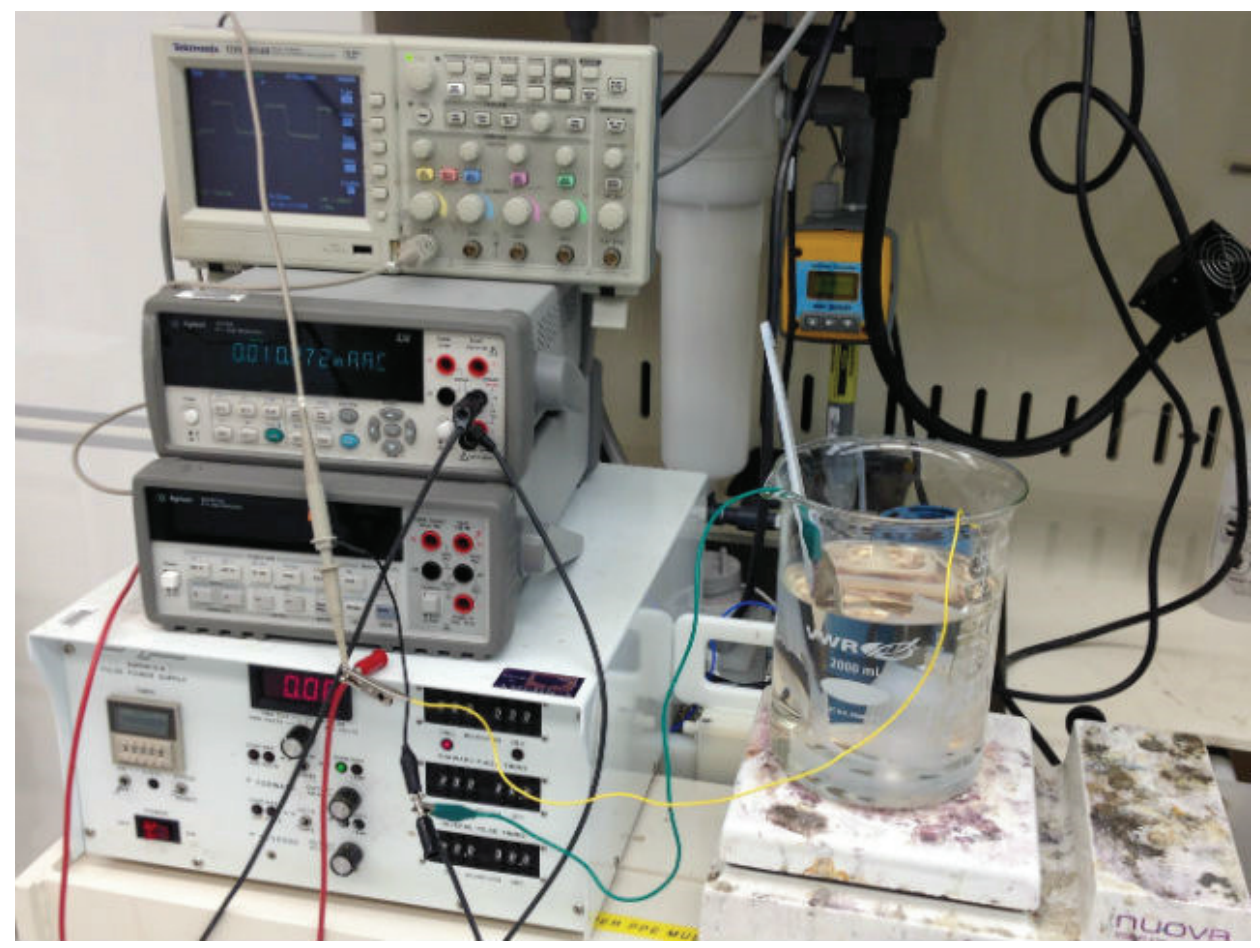

Figure 4.6 Silver electroplating system

The starting voltage was set to zero, then the power was turned on and the voltage was gradually increased until the desired current level was reached. A timer was set for one hour and the plating current was constantly monitored. In general, plating current rose as the hour progressed, up to approximately 30-40 $\mu \mathrm{A}$. After one hour, the wafer was taken out, washed with DI water and dried with the nitrogen gun. The wafer was placed in the Dektak 8 profilometer to measure the amount of silver grown in the electrode trenches. Typically, the trench was expected to grow from 2.5 to $3 \mu \mathrm{m}$ below the polyimide surface up to $\sim 200 \mathrm{~nm}$ above the surface. The extra silver ensured enough material was present after the chloridization process to guarantee electrode contact with the tissue.

During the first wafer fabrication runs, silver electroplating was unsuccessful. Some electrodes were plated while others were not (Figure 4.7). Attempts to clean the Silver Cy-less II solution were unsuccessful. It was discovered that the silver plating solution was over 2 years old, but the product lists a shelf life of only 6 months. Thus, new 
silver electroplating solution was purchased and the silver-plating fabrication step was no longer an issue. High quality, bright silver was seen using the new solution.

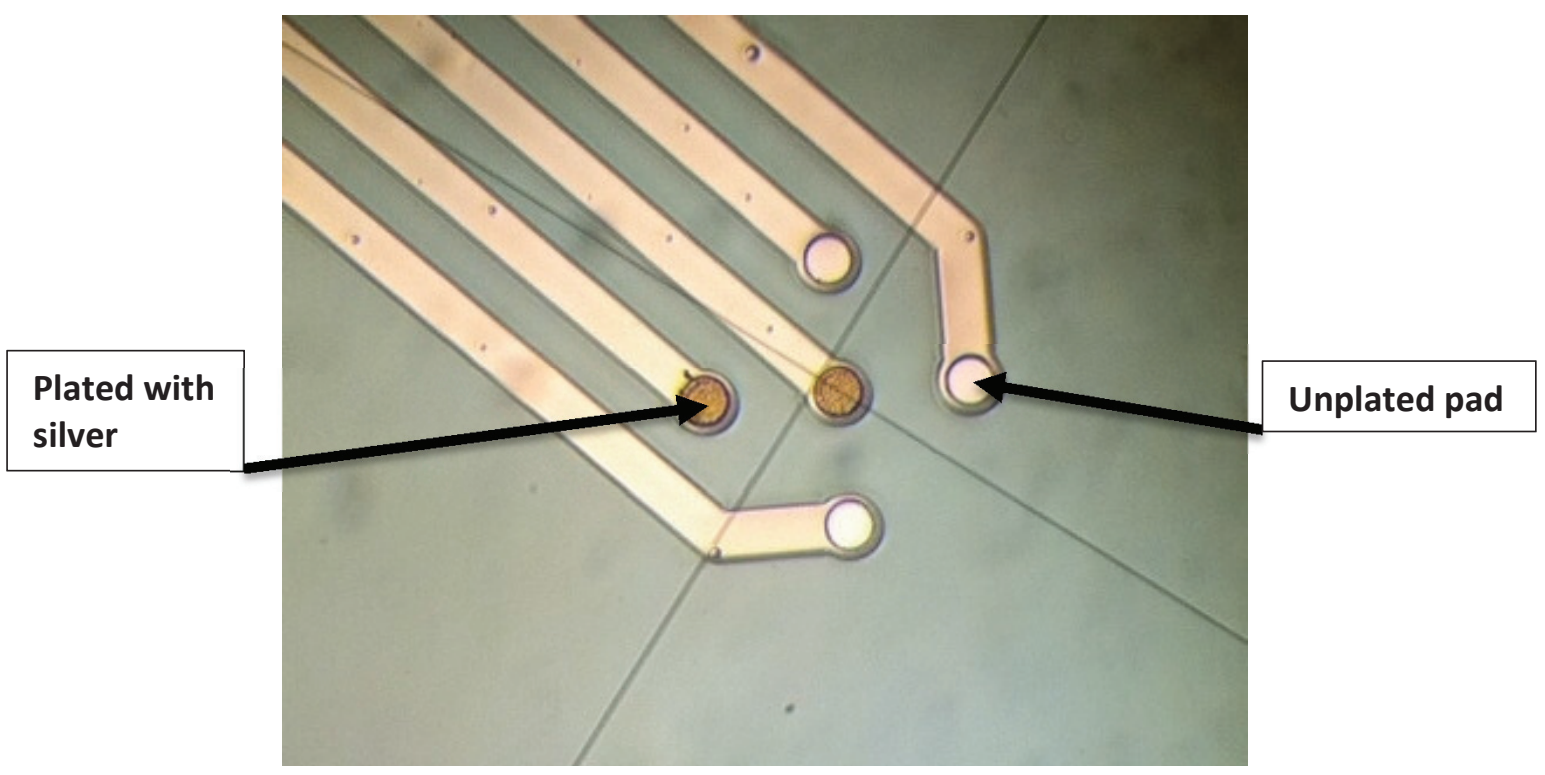

Figure 4.7 Silver electroplating deposition issues using old solution

After silver electroplating was successfully completed, the electrodes were chloridized. The chloridization process was similar to silver electroplating process except the polarity was reversed to attract the chloride ions to the electrode surface to react with the silver, creating a boundary of silver and silver/chloride to form. The wafer anode and platinum cathode were placed in $1 \mathrm{M}$ saline solution and a $100 \mathrm{~ms}, 40 \%$ duty cycle, $50 \mathrm{mV}$ pulsed DC waveform was run through the system for ten seconds. The height of the electrodes was once again measured to ensure that a few hundred nanometers growth occurred indicating the addition of chloride. The photoresist from mask three was stripped off with acetone. 


\subsection{Packaging}

The MEA sensors were removed from the wafer by first cutting the metal leads that provide an electrical connection to deliver current to each sensor for the electroplating and chloridization processes. Then each MEA sensor was very carefully cut with an X-Acto knife. A plastic reinforcement tab was created using a laser cutter. The plastic reinforcement tab was glued to the backside of the MEA sensor connector to provide structural rigidity and the necessary thickness to enable attachment of a ZIF clamp to hold the MEA sensor in place. After mounting the plastic reinforcement tab, the MEA bonding pads were aligned under a microscope with the bonding pads of the ZIF connector and the connector was carefully forced down, locking the MEA sensor connection pads to the 25pin connector. Care was taken to prevent misalignment due to MEA sensor fragility, since it was impossible to remove the plastic tab and realign without destroying the MEA. A five minute quick setting epoxy was found to give the best results in terms of allowing time to properly align the connector as well as providing structural integrity and withstanding the insertion forces into the connector.

\subsection{Testing and Characterization}

To verify that each of the 25 electrodes per MEA sensor were functional, the potential voltage difference between each electrode and a reference silver / silver chloride electrode was measured in $1 \mathrm{M}$ saline using a bench top $\mathrm{pH}$ meter in $\mathrm{mV}$ function. The MEA and reference electrode were placed together in a beaker with the saline. The reference electrode was connected to one of the $\mathrm{pH}$ meter leads and one of the electrodes from the MEA was connected to the other $\mathrm{pH}$ meter lead. If the MEA electrode was intact, had a good electrical connection, and had a viable layer of silver / silver chloride, the 
potential voltage difference between the two electrodes in $1 \mathrm{M}$ saline was near $0 \mathrm{mV}$. Differences greater than tens of millivolts indicated a bad electrode. This could be caused by a broken or damaged trace on the MEA, a misalignment or bad connection of the 25pin connector or improper chloridization or silver plating. In measuring each electrode, the entire MEA was characterized without damage to the electrodes themselves.

After the above verification, a conductive gel was made in order to test in vitro the performance of each MEA sensor before being used in vivo. A product manufactured by Oil Center Research called TX-151, known also as "The Improved Amazing Rubber Water with 1001 Uses" was used as a thickening agent to make a gel on which the TFMEA was placed. To make the conductive gel, one liter of water was heated to boiling point while being stirred with a magnetic bar on a hotplate. Next, 31 grams of agar was added and allowed to mix well. When the agar was fully mixed with the boiling water, 162.4 grams of sucrose was added and allowed to fully dissolve. The sucrose acted as the conductive medium for the gel and in conjunction with the TX-151 gel created a material with similar electrical and physical properties to cardiac tissue. Finally, 16.9 grams of the TX-151 powder was added. Care had to be taken to add the powder very slowly to prevent clumping of the powder. The rubberized clumps could not be broken apart and dissolved, so a new batch would have to be started if this occurred. Once the TX-151 powder was added, the solution thickened considerably and manual stirring was necessary. When the TX-151 was fully incorporated, the thick gel was poured into a flat plastic container and placed in a refrigerator to solidify.

After the gel was allowed to set up, large flat electrode plates were added to either side of the container. A voltage was applied across the plates to provide a potential gradient 
across the gel to be measured by the TFMEA. A cardiac pacer and an Agilent function generator with cardiac function were used separately to verify signals of different types. The pacer provided a square wave like signal similar to a cardiac action potential while the Agilent provided a simulated ECG signal. The TFMEA was placed on the gel perpendicular to the metal plates, in the middle of the gel (Figure 4.8).

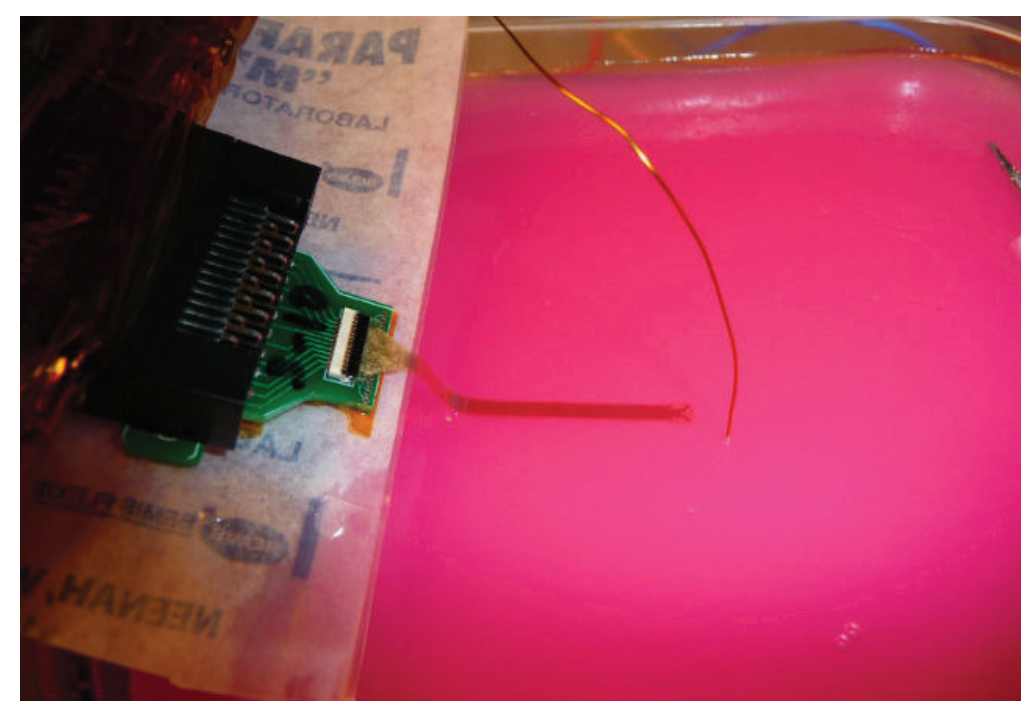

Figure 4.8 In vitro characterization of TFMEA on conductive gel

A silver / silver chloride reference electrode was placed in the gel within a few centimeters of the TFMEA, saline was added to improve the interface between the TFMEA and the gel surface. The closer the reference electrode to the TFMEA, the greater the amplitude of the resultant signal from the signal conditioning board. The signal conditioning microchip (see description below) was connected to the NI-DAQ and to the TFMEA and the reference electrode was connected to the ground of the SCM as well as the DAQ. Both metal plates in the gel were connected to their respective output and turned on. Data was recorded for later analysis using National Instruments Signal Express software at a rate of $16 \mathrm{kHz}$ per channel, using the USB-6353 DAQ. 


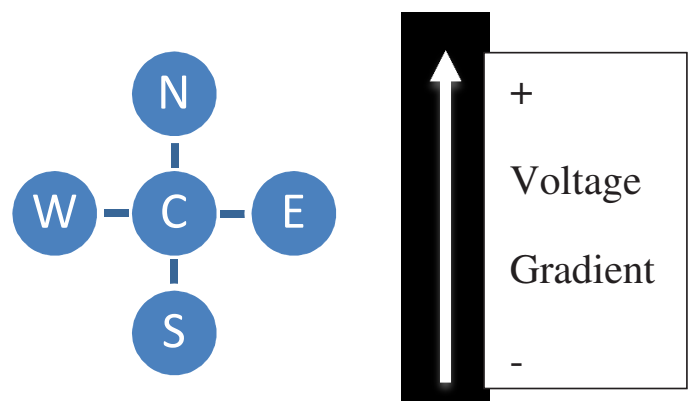

Figure 4.9 One group of five electrodes, showing the center, north, south, east, and west pads

If a voltage gradient is applied from south to north as shown in Figure 4.9, then the west, center, and east electrodes will see the same potential, while north will see a more positive potential and south will see a more negative potential. The surface Laplacian is calculated in the following manner:

$$
S L=\frac{4 * V_{C}-V_{W}-V_{N}-V_{E}-V_{S}}{d^{2}}
$$

Where $\mathrm{V}_{\mathrm{C}}$ is the voltage at the center electrode, $\mathrm{V}_{\mathrm{W}}$ is the voltage at the west electrode, $\mathrm{V}_{\mathrm{N}}$ is the voltage at the north electrode, $\mathrm{V}_{\mathrm{E}}$ is the voltage at the east electrode, and $\mathrm{V}_{\mathrm{S}}$ is the voltage at the south electrode. The variable $\mathrm{d}$ is the distance from electrode to electrode, which is $75 \mu \mathrm{m}$.

\subsection{New Signal Conditioning Board}

Due to problems with the original signal conditioning circuit board, a new signal conditioning circuit board was designed to interface the microelectrode array to a signal acquisition device. The new design focused on the use of the Texas Instruments LMP770x series of operational amplifiers. These were precision amplifiers packaged in one, two, and four channel versions. The LMP770x series was particularly attractive when paired with a microelectrode array. Often the electrical resistance at the interface between tissue and sensor was very high, limiting the amount of bias current available to drive the input of the 
amplifier. In order to obtain the largest possible input voltage without loading down the electrode, an amplifier with very low input bias current was selected. The LMP770x series was specifically designed for this application and has an extremely low input bias current, less than $\pm 1 \mathrm{pA}$. These chips also had very low noise and offered a large gain-bandwidth. The LMP770x series was operated from a DC power supply in the range of $2.7 \mathrm{~V}$ to $12 \mathrm{~V}$ with a low supply current of $2.9 \mathrm{~mA}$ (four channels), making this device ideal for batterypowered applications.
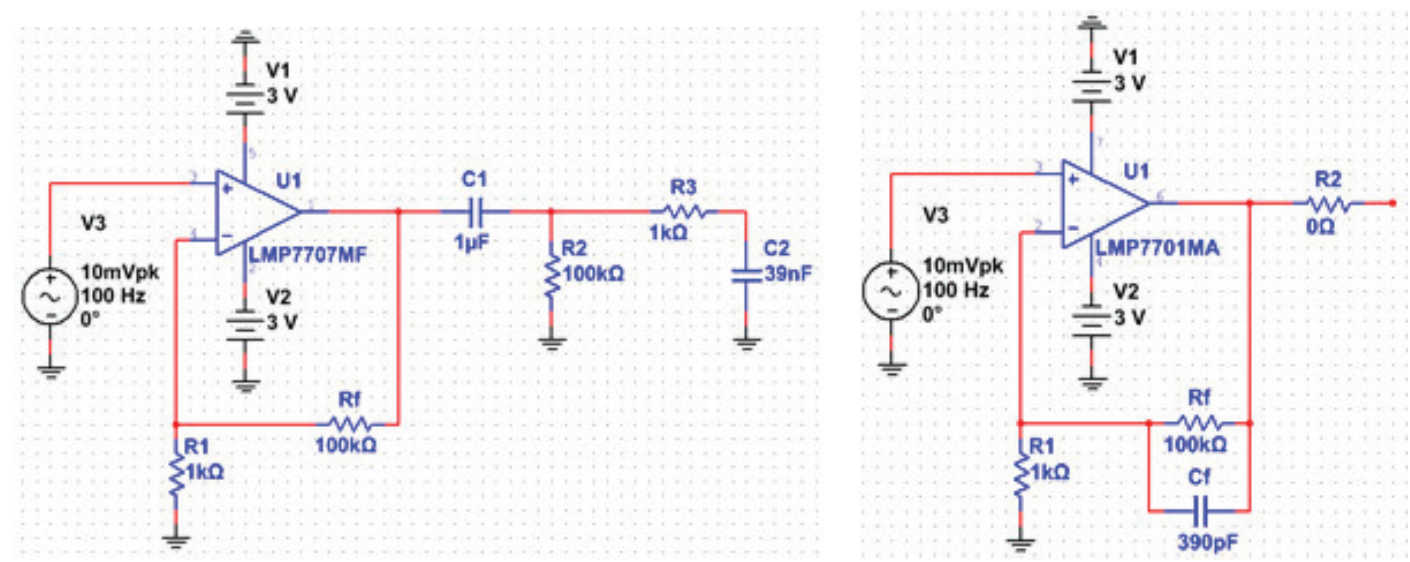

Figure 4.10 Schematics used in the new signal conditioning board

Shown on the left in Figure 4.10 is the schematic for the first signal conditioning design. A passive band-pass filter was chosen over an active design because it was important to not load down the microelectrode array at the input of the amplifier. Because of this, the signal path from the MEA ran directly into the op-amp non-inverting input. Components $\mathrm{C} 1, \mathrm{C} 2, \mathrm{R} 2$, and $\mathrm{R} 3$ make up the passive filter, which was designed so that the high-pass cutoff point of the filter occurred at $1.5 \mathrm{~Hz}$ and the low-pass cutoff point of the filter occurred at $4 \mathrm{kHz}$.

Where possible, components values were made to be the same so that identical value parts could be used in several different locations in the design. This approach enabled 
the ordering of parts in bulk, which was cheaper and easier, and also aided in reducing errors when assembling the device. Since the output of the passive filter is connected to a high impedance analog to digital converter (10 G $\Omega$ ) input, loading down of the passive filter was near zero causing no additional voltage drop or frequency response change from the design specifications.

Shown on the right in Figure 4.10 is a secondary design that was DC coupled. This design used a small capacitor in the feedback loop to create the $4 \mathrm{kHz}$ low-pass filter instead of relying on the passive filter. The passive filter section was removed entirely. Only one of the three boards was populated using this design primarily to verify functionality versus the passive filter board design. This design used the LMP7701/7704, which was stable at unity gain. Unity gain stable refers to an amplifier that is stable when used with a gain equal to one, in which the amplifier acts simply as a buffer. This was important because the LMP7707/7709 was not unity gain stable and therefore could not be used in the secondary design because above $4 \mathrm{kHz}$ the gain of the op-amp approached 1 (unity) and progressed there well before the total bandwidth of the op-amp (14MHz). If the LMP7707/7709 were used with the feedback capacitor, it would be unstable. 


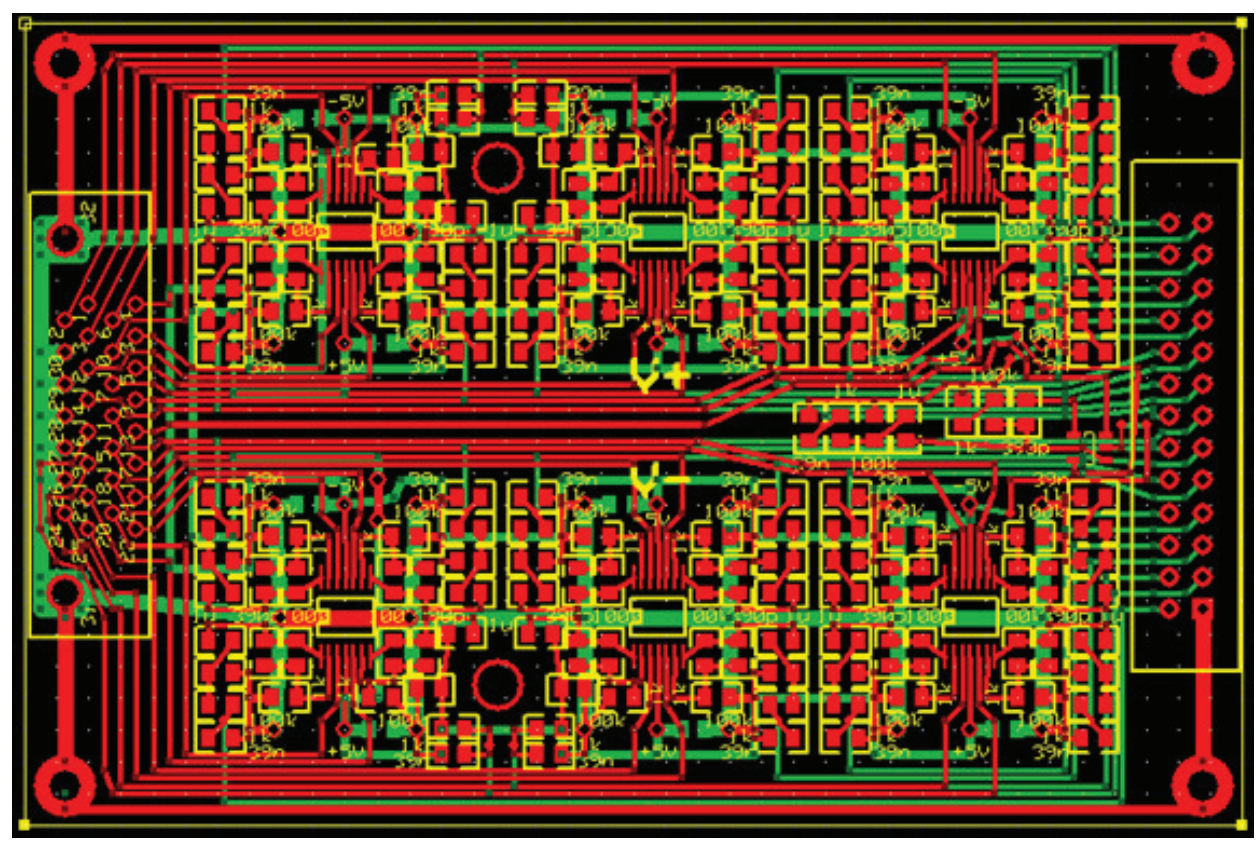

Figure 4.11 Final printed circuit board of new design

The new board was designed to fit in the same physical footprint as the original design and use the same number of input and output connectors while offering better analog electrical performance and physical reliability than the original design. ExpressPCB was chosen as the PCB manufacturer because of fast turnaround time in addition to providing easy to use CAD software. Total board space available was 3.8 " by 2.5 ". Using this service, three high quality silkscreened boards were manufactured and delivered within four business days for $\$ 70$. Figure 4.11 showed the final layout of the printed circuit board in ExpressPCB. The red layer was the top copper layer, the green layer was the bottom copper layer, and the white layer was the silkscreen used to display component positioning and value information. All surface mount components, except for 12 decoupling capacitors, were placed on the top layer of the board. Two large through holes were placed near the middle of the board to allow for placement of screws to hold a 4 x AAA battery holder. The schematic for battery connection was shown in Figure 4.12. Vec is $3 \mathrm{~V}$ and Vdd is $3 \mathrm{~V}$, with $0 \mathrm{~V}$ ground referenced in the middle of the four AAA batteries. With four fresh 
alkaline batteries, this design had a battery life of approximately 50 hours of continuous use.

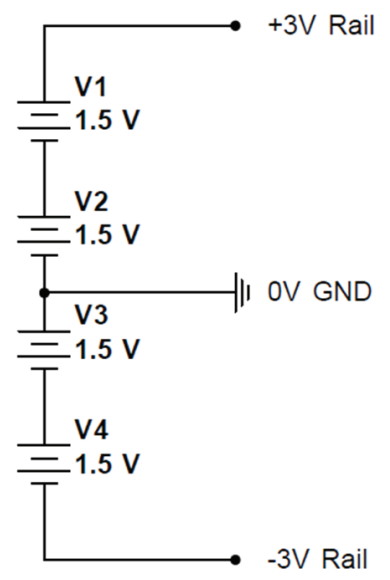

Figure 4.12 Battery connection schematic

Once the newly manufactured printed circuit boards were populated, each channel of each board was characterized for gain, frequency response, noise, and DC offset. Individual sine waves of $10 \mathrm{mV}$ amplitude were input to all channels simultaneously and the output measured by the National Instruments USB-6353 DAQ. Sine waves of $1 \mathrm{~Hz}, 10$ Hz, $100 \mathrm{~Hz}, 1000 \mathrm{~Hz}, 2000 \mathrm{~Hz}, 3000 \mathrm{~Hz}$, and $4000 \mathrm{~Hz}$ were used. Recordings were also made with all inputs tied to $0 \mathrm{~V}$ so that a baseline noise measurement could be acquired. 


\section{RESULTS AND DISCUSSION}

Once fabrication and packaging had been completed, the individual MEAs were tested to ensure all channels were functional. Electrical resistance measurements were carried out on a small number of electrodes, because this effectively ruined the entire MEA by damaging all electrodes tested. All channels on all MEAs were checked for continuity using an electrochemical method that did not harm the delicate silver chloride cap. Once the MEA passed the continuity check it was placed on a conductive gel used for in vitro verification. After completing in vitro testing the MEAs were washed and stored until used for in vivo testing.

\subsection{TFMEA Metrology and Electrical Resistance Measurements}

Reduction in resistance of the metal traces was measured using a probe station and digital multimeter and the comparison in resistances between old and new MEAs is shown in Table 5.1 below. Resistance of the original Ti/Pt traces was slightly higher than the theoretical predicted values derived in Chapter 4, most likely due to small variances in the actual amount of metal ( $\mathrm{Ti}$ and $\mathrm{Pt}$ ) deposited on the polyimide thin film. However, the resistances of the $\mathrm{Ti} / \mathrm{Ag} / \mathrm{Pt}$ traces were very close, within $\pm 7 \Omega$, to the theoretical calculations. Thus, the addition of the third metal layer of silver did significantly reduce the resistance by one order of magnitude. 
Table 5.1 Comparison of trace resistances

\begin{tabular}{|c|c|c|}
\hline Trace & $\begin{array}{l}\text { Resistance } \\
\text { Ti/Pt }(\mathbf{\Omega})\end{array}$ & $\begin{array}{l}\text { Resistance } \\
\text { Ti/Ag/Pt }(\mathbf{\Omega})\end{array}$ \\
\hline $\mathbf{1}$ & 1500 & 92.4 \\
\hline $\mathbf{2}$ & 1040 & 89.6 \\
\hline $\mathbf{3}$ & 1130 & 90.6 \\
\hline $\mathbf{4}$ & 1068 & 94.5 \\
\hline $\mathbf{5}$ & 1135 & 94.0 \\
\hline MEAN & $\mathbf{1 1 7 5}$ & $\mathbf{9 2 . 2}$ \\
\hline STDEV & $\mathbf{1 8 6}$ & $\mathbf{2 . 1}$ \\
\hline
\end{tabular}

During the silver electroplating step, careful records of the electrode trench depth were kept before, during, and after the silver electroplating, silver chloridization, and annealing processing steps. Table 5.2 shows a summary of this data from two different silicon wafers during fabrication. Both wafers initially had electrode trenches measuring 3 $\mu \mathrm{m}$ below the polyimide surface prior to electroplating. After electroplating was finished, it was desirable to have material plated slightly above the surface to ensure the microelectrodes are in direct contact with the cardiac tissue. This was achieved as indicated by the positive values of $325 \mathrm{~nm}$ and $528 \mathrm{~nm}$ post processing of the silver electroplating step. After silver chloridization was complete, as expected, another growth had occurred due to the addition of chloride to the silver, but the $40-100 \%$ increase was not anticipated. While silver electroplating was a relatively time consuming process which took on the order of two hours to complete, silver chloridization was a very quick process. In $1 \mathrm{M}$ saline, the silver plated electrodes were chloridized in a matter of seconds. It was important to keep voltages and currents low (less than $100 \mathrm{mV}$, less than $20 \mu \mathrm{A}$ ) to avoid over chloridization. In the case of a $40-100 \%$ increase in electrode height it may have been possible that the electrodes were over chloridized. This was an issue because too much 
silver chloride caused the fragile electrode cap to break off easily, destroying the electrode altogether.

Interestingly, after annealing at $450{ }^{\circ} \mathrm{C}$ for two hours, another growth was seen, $14 \%$ for wafer 1 and $36 \%$ for wafer 2 . One possibility for this increase could be attributed to some of the chloride diffusing further into the electroplated silver. Another possibility could be the flow of the polyimide material from the sidewalls of the well into the electroplated silver due to the annealing temperature being above the glass transition temperature of the polyimide. The Dektak measurements also indicated that annealing increased surface uniformity on the electrodes, except for near the edges of the electrodes, where large spikes of material had formed. It was possible that when the wafer was removed after two hours at $450{ }^{\circ} \mathrm{C}$ the silver chloride layer had at least partially melted, since its melting temperature is $455^{\circ} \mathrm{C}$. Once the wafer was taken out of the furnace it was quenched rapidly, perhaps some of the silver chloride which had melted solidified into the spikes that were seen.

Table 5.2 Depth of electrode trench, before and after silver electroplating, chloridization, and anneal

\begin{tabular}{|l|c|c|c|c|}
\hline Depth (nm) & Pre-AgEP & Post-AgEP & Post-AgCl & Post-Anneal \\
\hline WAFER 1 & -3000 & 325 & 657 & 750 \\
\hline STDEV & & 137 & 69 & 168 \\
\hline WAFER 2 & -3000 & 528 & 764 & 1040 \\
\hline STDEV & & 284 & 341 & 129 \\
\hline
\end{tabular}

\subsection{Preliminary In vitro Characterization of New TFMEA with previous SCM}

A sample of data taken from an improved TFMEA using the previous SCM system

on the conductive gel is shown in Figure 5.1. The external cardiac pacer was connected to the metal plates on either side of the conductive gel. The results of this in vitro testing were 
thought to be adequate in order to proceed with in vivo testing. Baseline noise seemed negligible compared to the signal of interest. However, ECG signals collected from the in vivo experiment were of much lower magnitude than what was seen in this gel experiment and the electrical noise became an issue. After revisiting these in vitro results, the same baseline noise was seen that was found during the in vivo experiment.

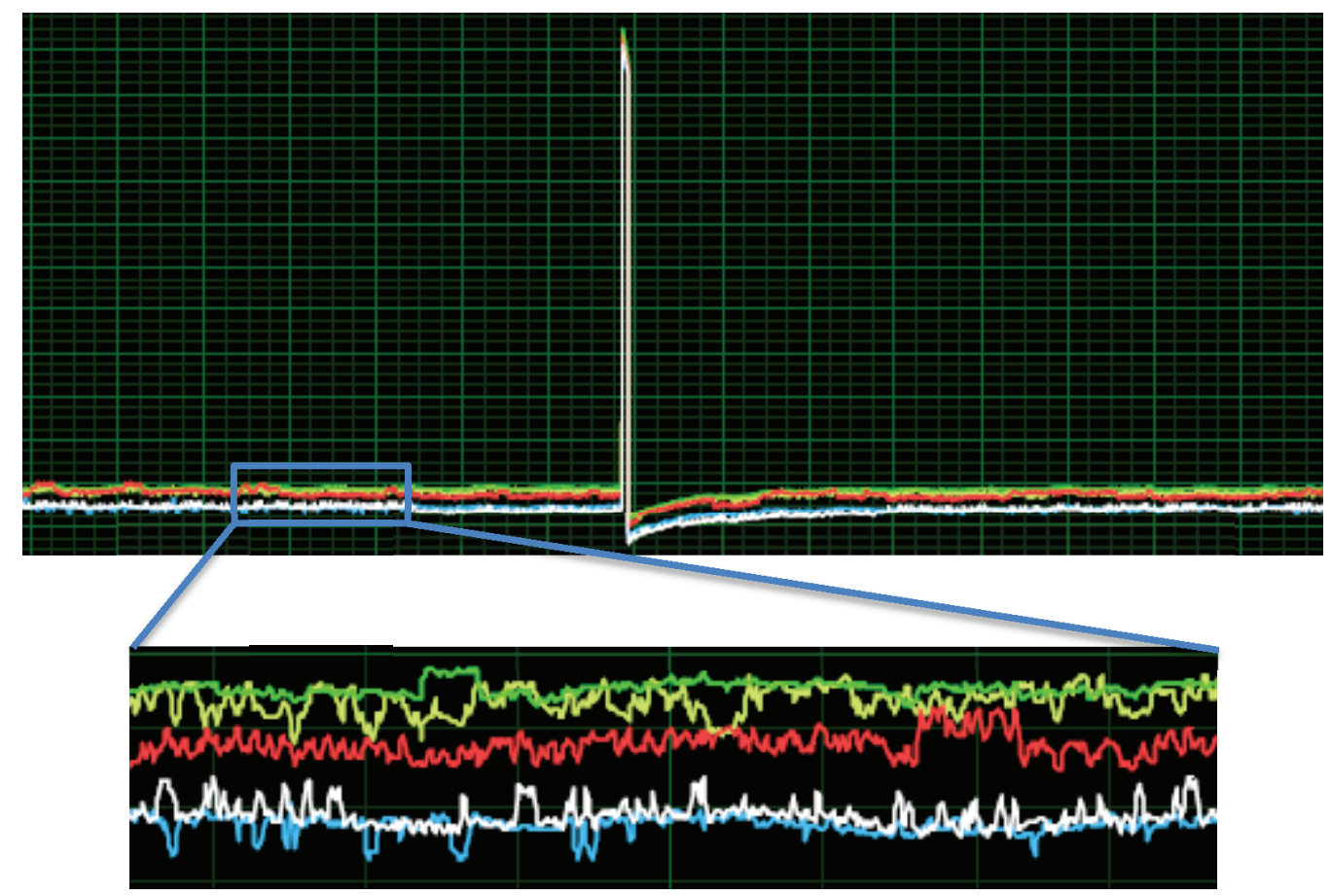

Figure 5.1 In vitro gel characterization of improved TFMEA with SCM, electrical noise shown below

\subsection{Preliminary In vivo Characterization of New TFMEA with previous SCM}

The first set of in vivo data was collected at the University of Utah on a rabbit heart and a substantial amount of electrical noise was noted. This noise appeared as distinct jumps in the output from the signal conditioning microchip as seen prominently in the blue ECG signal in Figure 5.2 below. The signal-to-noise ratio of this signal was $22.47 \mathrm{~dB}$. This noise was so great in magnitude compared with the actual ECG signal that it could not be 
effectively filtered out without removing important details of the ECG signal. Since the surface Laplacian data was generated from a complex combination of a group of five electrodes at one time, this noise prevented extraction of any useful information. Figure 5.3 shows the noise in more detail as seen on a Tektronix oscilloscope.

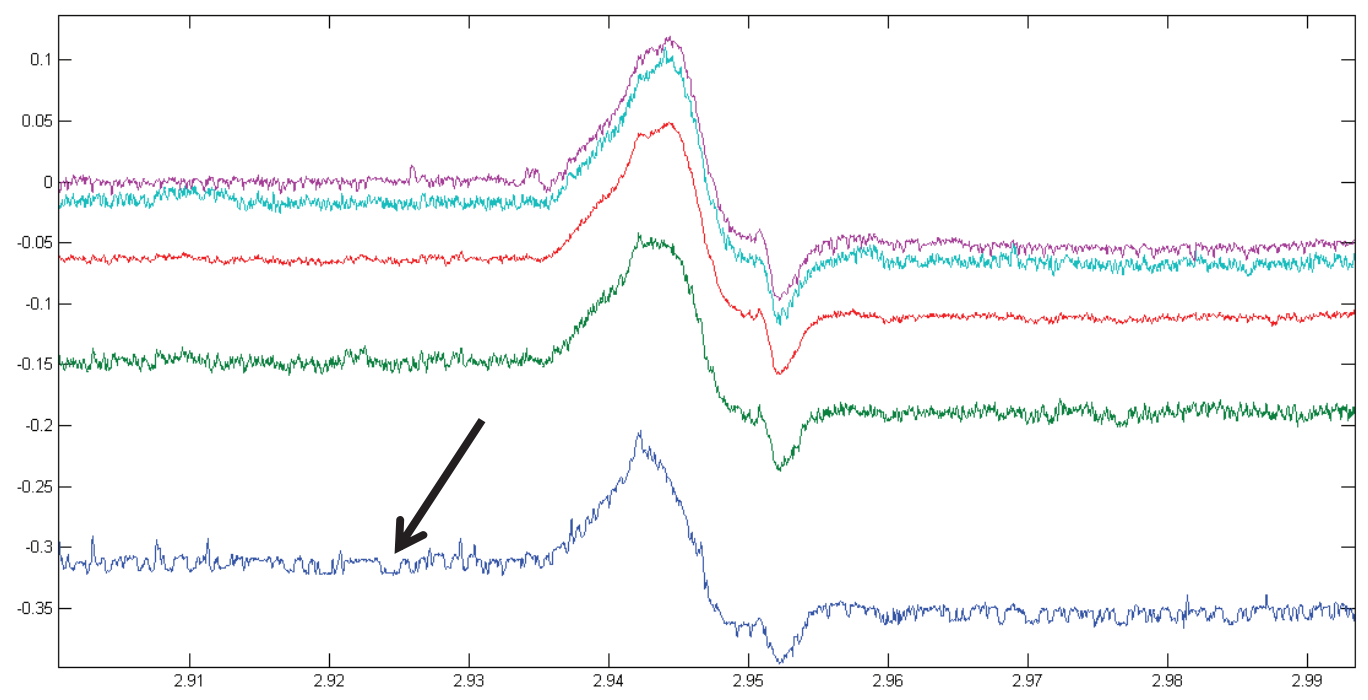

Figure 5.2 Collection of ECG from surface of rabbit heart, one group of five electrodes. Arrow shows distinct jump.

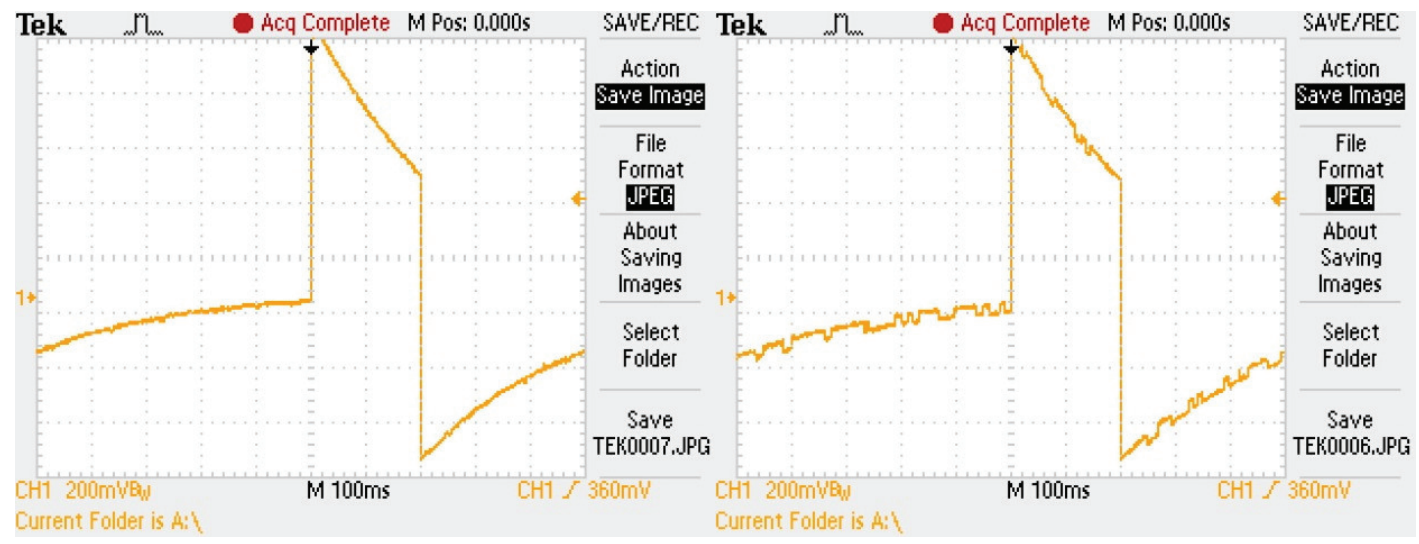

Figure 5.3 Clean signal on left, noise on right using a Tektronics oscilloscope

Since the signal conditioning microchip used MOSIS custom integrated circuits containing an array of operational amplifiers, it was possible that these chips could have deteriorated or had weak or failing output transistors. From the original MOSIS run, there 
were an additional 30 die, which had not been packaged into the PGA-121 ceramic package. These die were sent to a packaging and test company called CORWIL, located in Milpitas, CA. CORWIL packaged 25 of the 30 die. Upon arrival, the new packaged chips were tested and characterized. An Agilent arbitrary function generator was connected to all channels of the SCM and each output of the SCM was wired to an analog input channel on the NI DAQ. Sine, square, and simulated ECG signals of $10 \mathrm{mV}$ and varying frequency from $0.5 \mathrm{~Hz}$ to $4 \mathrm{kHz}$ were placed at the input of the SCM.

Unfortunately the issue was still present. In addition to packaging new devices, a blank circuit board was populated using new components and the newly packaged MOSIS chips. The above tests were performed again with new microchips and new circuit board. Again, the noise issue was present. This issue was present using the National Instruments USB-6353 DAQ as well as when viewed exclusively with the Tektronix oscilloscope. Since the noise was present with the newly packaged chips and newly constructed printed circuit board, the problem seemed to lie with the MOSIS die themselves. A major disadvantage to using custom fabricated integrated circuits is that the amount of troubleshooting and repair that can be performed is minimal compared to COTS components, which can be depopulated and replaced.

\subsection{SCM Re-characterization}

The SCM was re-characterized before designing a new signal conditioning board. All input channels were connected to a function generator. The outputs of the SCM were connected to the USB-6353 DAQ. This allowed for the calculation of key amplifier 
parameters such as gain, DC offset, frequency response, and signal to noise ratio. These parameters are shown in Figures 5.4 to 5.7 below.

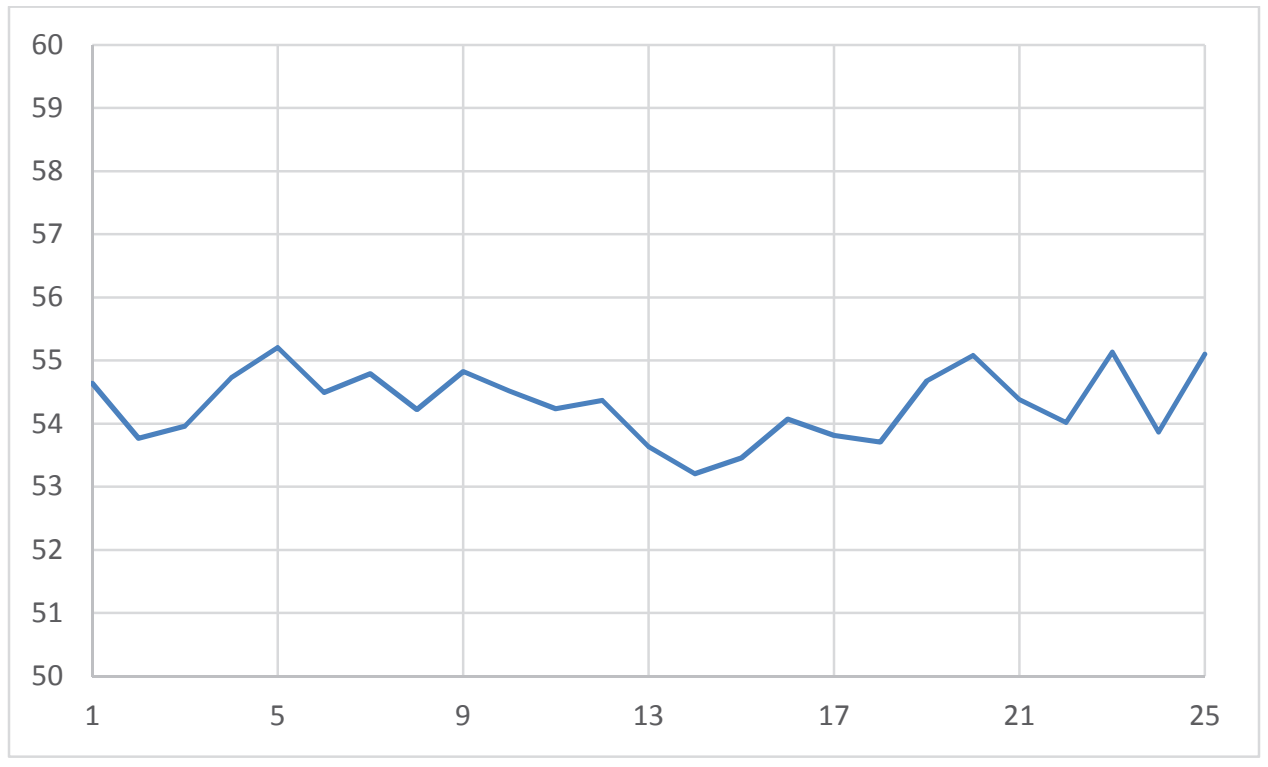

Figure 5.4 SCM gain (dB) versus channel. Average 54.3 V/V.

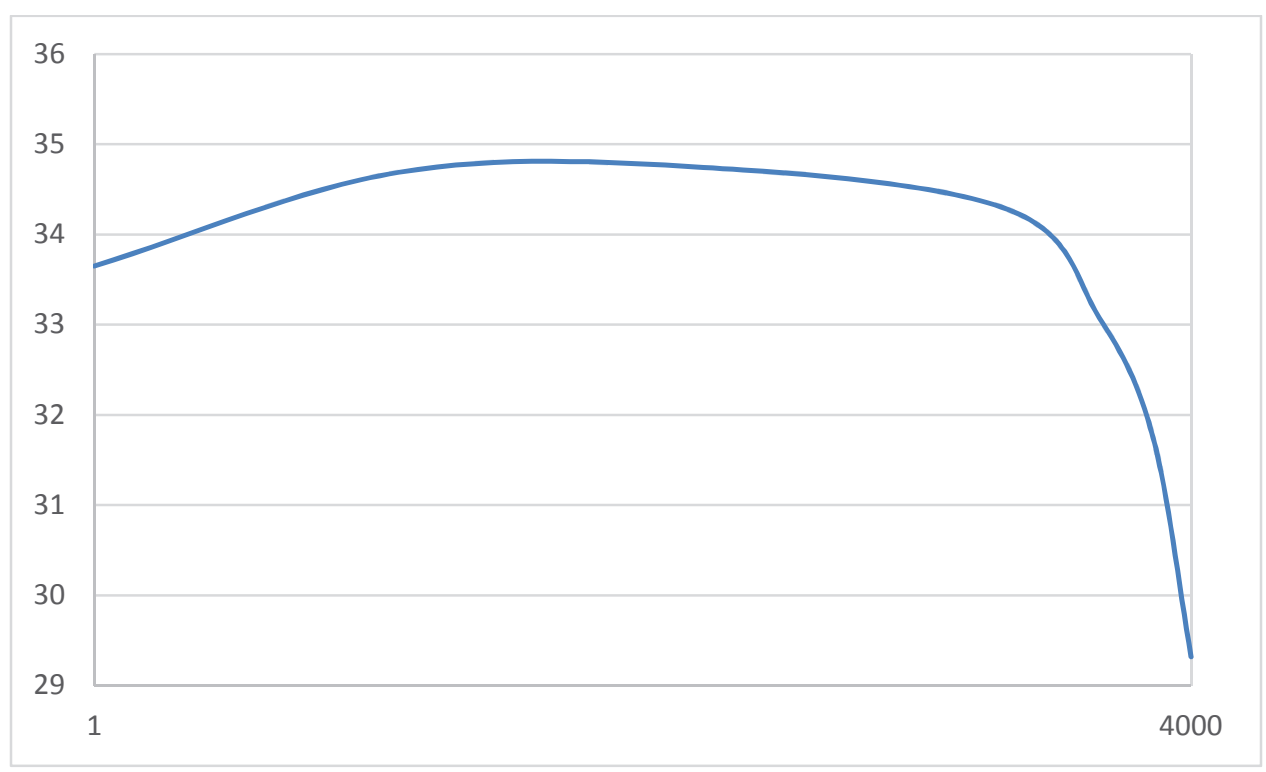

Figure 5.5 SCM frequency response (dB) versus channel. 


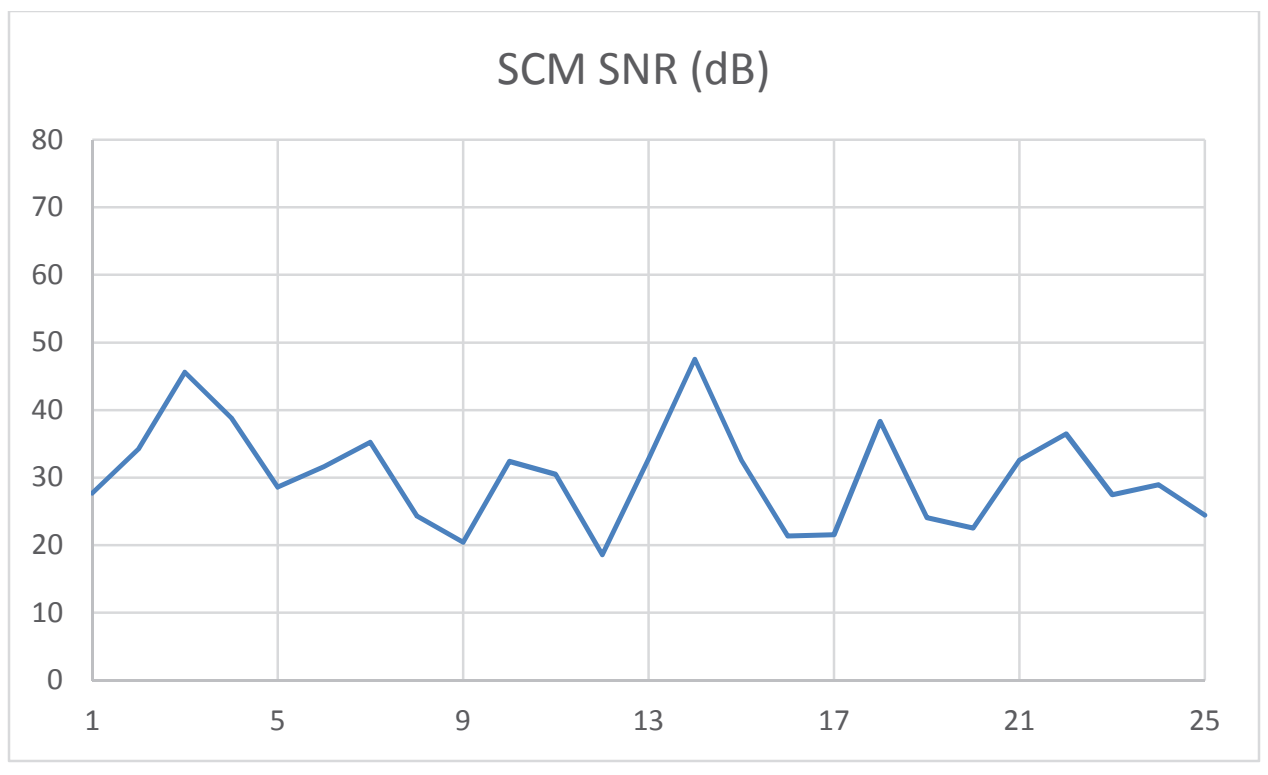

Figure 5.6 SCM signal to noise ratio (dB) versus channel. Average $30.4 \mathrm{~dB}$.

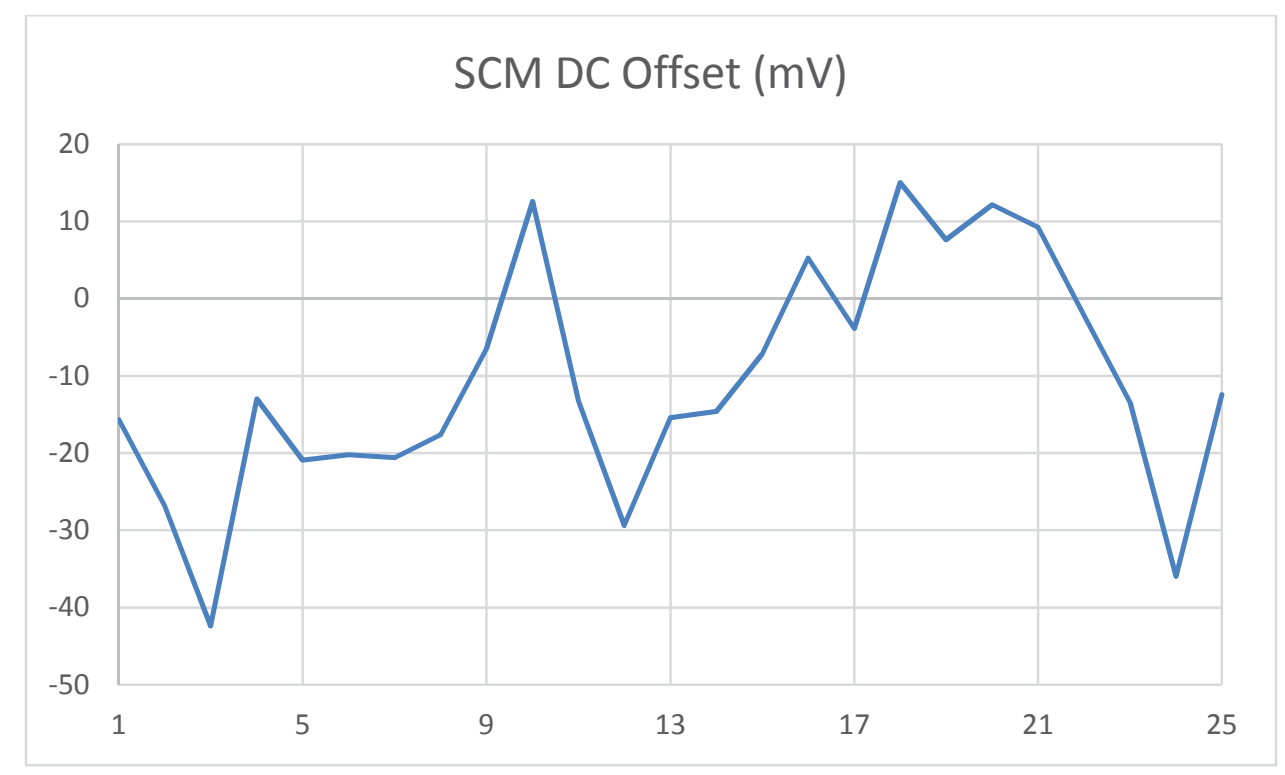

Figure 5.7 SCM DC offset $(\mathrm{mV})$ versus channel. Average $\mathbf{- 1 0 . 8} \mathrm{mV}$. Average gain was found to be $54.3 \mathrm{~V} / \mathrm{V}$ and deviated by approximately $+/-1 \mathrm{~V} / \mathrm{V}$ per channel. The signal to noise ratio was on average $30.4 \mathrm{~dB}$, with a minimum of $20 \mathrm{~dB}$ and a maximum of $47 \mathrm{~dB}$. DC offset for all channels averaged $-10.8 \mathrm{mV}$ but had a wide variation, with a maximum of $-42 \mathrm{mV}$. 


\subsection{New Signal Conditioning Board (SCB) Fabrication, Testing and Characterization}

In order to acquire high quality, low noise electrocardiogram data without a long lead-time and to avoid the high cost of designing and fabricating a new board, a new signal conditioning board (SCB) was developed. Instead of using a custom MOSIS design which takes months to manufacture, costs thousands of dollars, and which lacks the necessary ruggedness or performance, a custom-designed circuit board populated with commercialoff-the-shelf amplifiers was used to develop a new SCB. The off-the-shelf components were immediately available, low cost and possessed the electrical performance required for this microelectrode application.

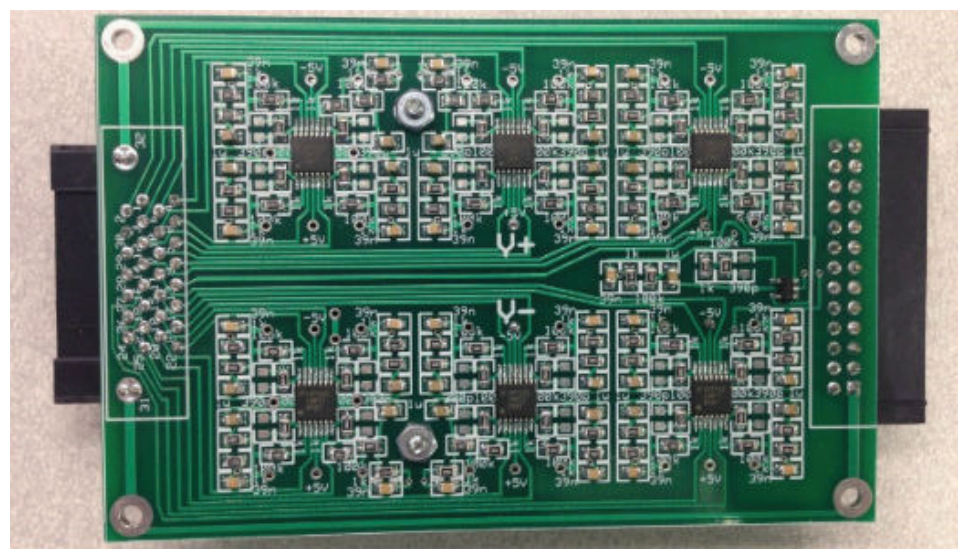

Figure 5.8 Bottom of new signal conditioning board

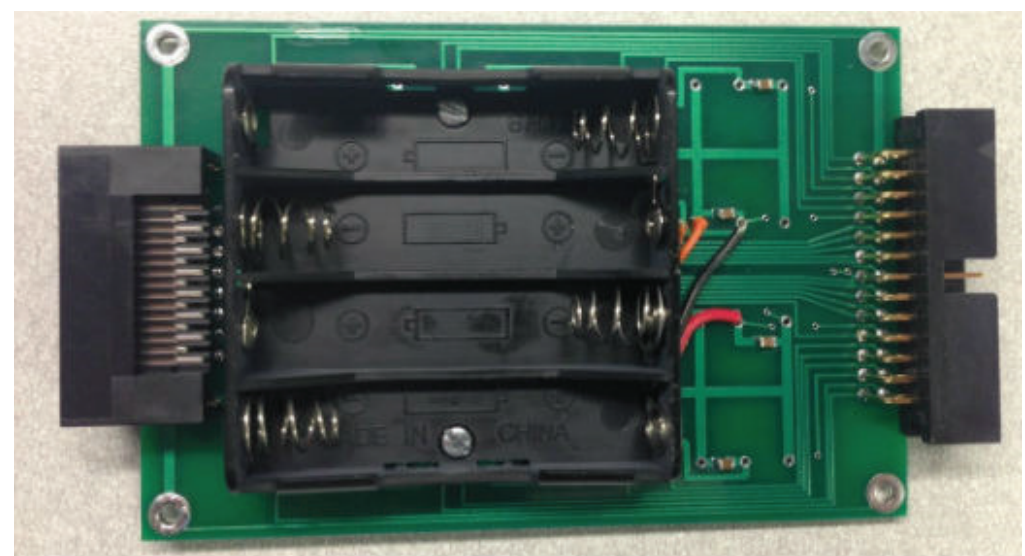

Figure 5.9 Top of new signal conditioning board 
Figures 5.8 and 5.9 show the final assembled printed circuit board. In total, three SCBs were built with standard surface mount packages: 0805 for resistors and capacitors, TSSOP-14 for quad channel op-amps, and SOT-23-5 for single channel op-amps. A 4 x "AAA" battery holder was placed on the top of the board and soldered to the positive and negative voltage rails of the board, with $0 \mathrm{~V}$ ground tied between the center two batteries. Final gain calculations were taken from data at $100 \mathrm{~Hz}$, representing the highest overall gain in the pass-band region of the amplifier (Figure 5.10). The design parameters were set to obtain a voltage gain of $100 \mathrm{~V} / \mathrm{V}$ and the actual gain achieved was on average $97 \mathrm{~V} / \mathrm{V}$ ( $3 \%$ of design specification). Deviation from channel to channel was less than $\pm 1 \mathrm{~V} / \mathrm{V}$ ( $1 \%$ compared to total gain). It was more important to achieve lower channel to channel deviation than to achieve gains that were closer to the design specification. This was because the surface Laplacian is highly dependent on the amplitudes of a group of channels, and not one individual channel.

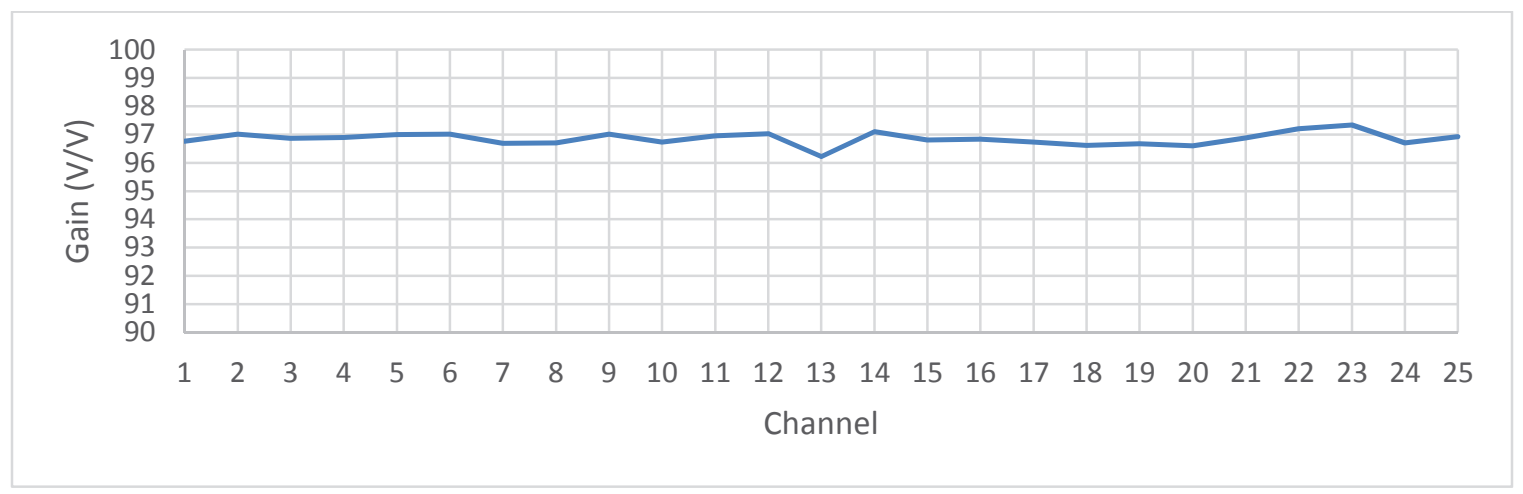

Figure 5.10 Gain measurements for all channels acquired for new SCBs

The average frequency response of all channels is shown in Figure 5.11, which illustrated that the actual frequency response was very close to the design parameters. This 
placed the $-3 \mathrm{~dB}$ pass-band between $1.5 \mathrm{~Hz}$ and $4 \mathrm{kHz}$. The signal to noise ratio (SNR) of each channel was found to average around $80 \mathrm{~dB}$ (Figure 5.12), a large improvement over the MOSIS design. Achieving a SNR of $80 \mathrm{~dB}$ allows the data acquisition system greater ability to resolve very small details in a signal, making this parameter a key point to optimize for ECG applications.

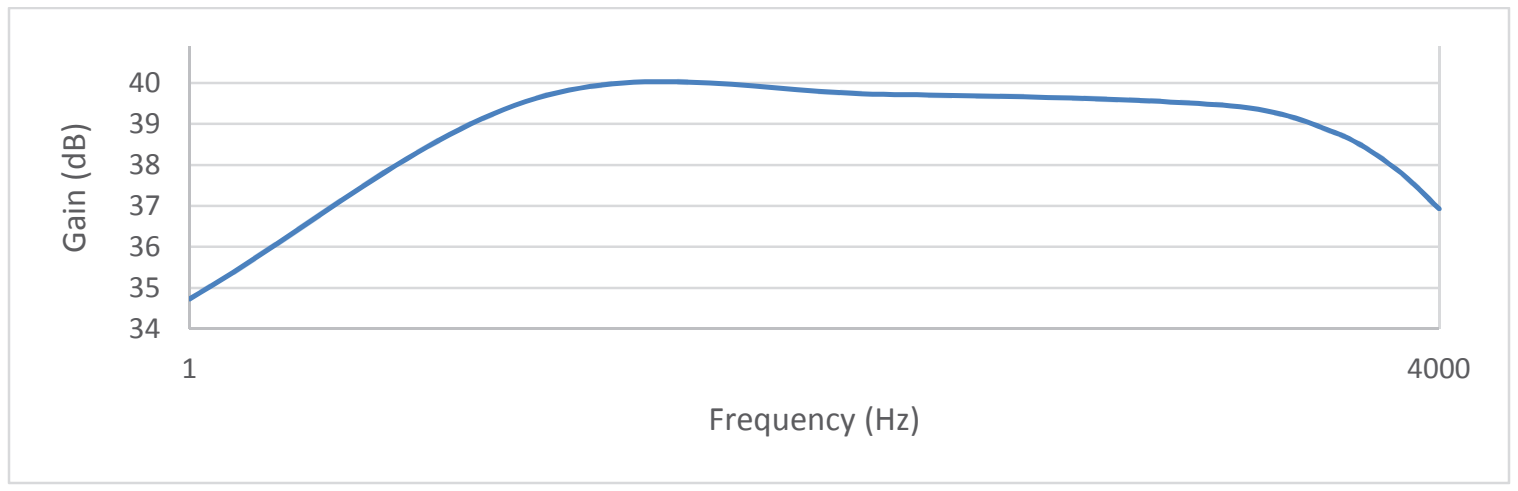

Figure 5.11 SCB Average Frequency Response (1 Hz to 4 kHz)

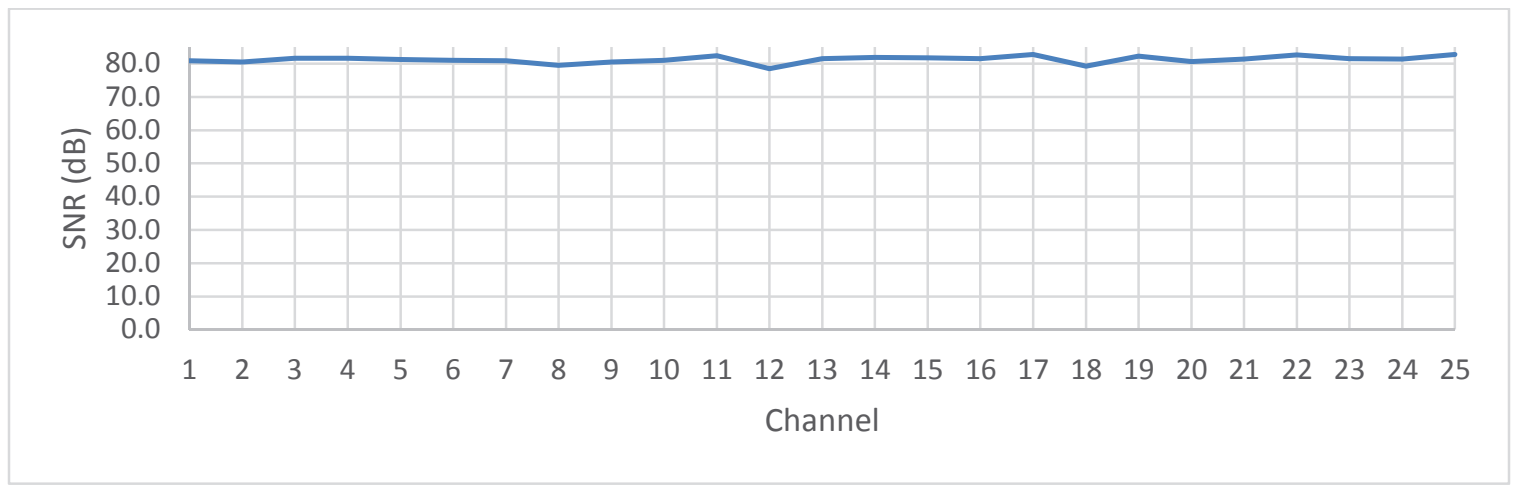

Figure 5.12 SCB Signal to Noise Ratio

Finally, the DC offset of each channel was generally found to be less than $500 \mu \mathrm{V}$ (Figure 5.13). This was also an improvement over the MOSIS design, which commonly had offsets of 10-20 mV. The DC offset was another very important parameter to minimize 
in this TFMEA application. Again, since the surface Laplacian is dependent on the combination of several channels, when each channel has a large offset compared to the features of the SL it greatly impacts the accuracy of the SL.

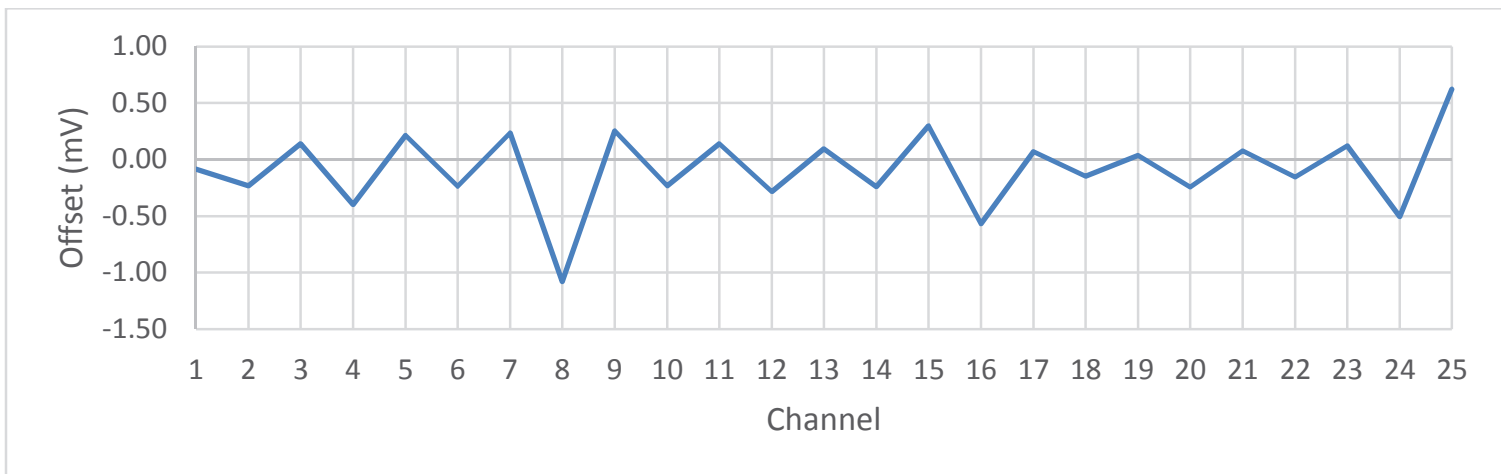

Figure 5.13 SCB DC Offset

Figures 5.10 through 5.13 illustrated that the new SCB closely matched design specifications. The new SCB coupled with the improved TFMEAs provided the necessary performance to proceed with in vitro testing. The results of in vitro testing with the new SCB are provided in section 5.7 below. TFMEAs used in the preliminary in vitro and in vivo study were not reused. To ensure maximum durability and signal quality, a new batch was fabricated for the experiments described in the sections below.

\subsection{Comparison of SCM versus SCB Performance}

A one-way analysis of variance was performed using Minitab 16 on the signal to noise ratio data obtained from the SCM and the SCB. The ANOVA plots are shown in Figure 5.14. The Minitab ANOVA results are shown below for a confidence level of 95\%. These results indicate that there is a significant $(\mathrm{p}=0.000)$ difference in the signal to noise 
ratios of the SCM versus the SCB. The R-squared value of greater than $95 \%$ indicates a good fit and the ANOVA plots in Figure 5.14 appear normal.

\section{One-way ANOVA: C12 versus C13}

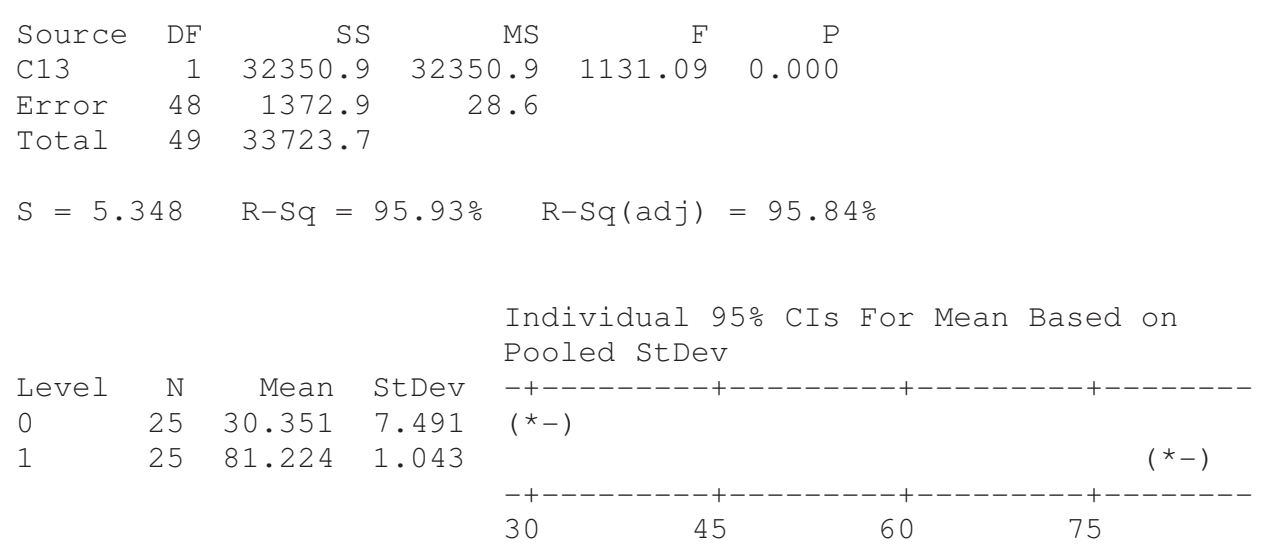

Pooled StDev $=5.348$

\section{Residual Plots for C12}
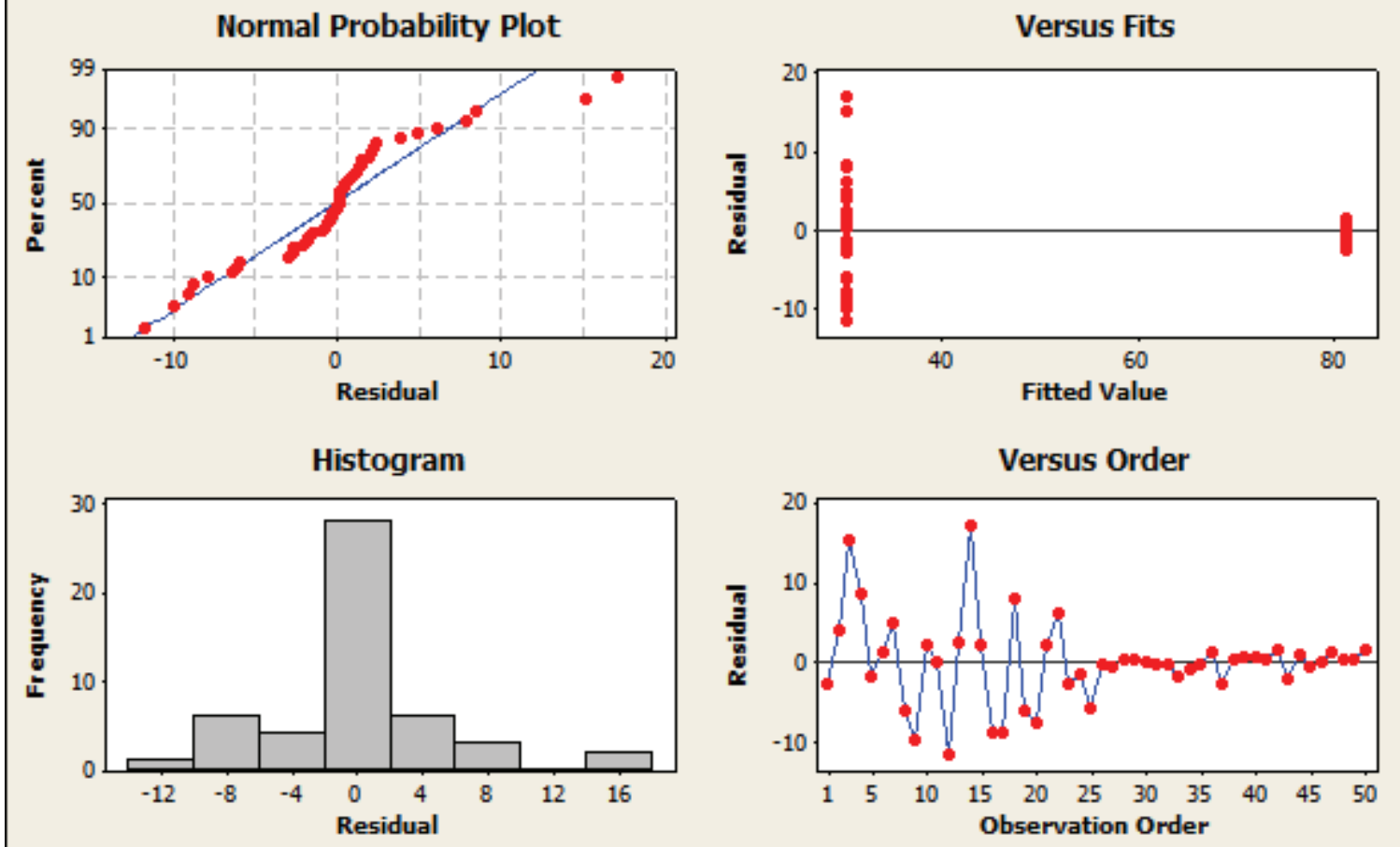

Figure 5.14 ANOVA plots based on SNRs of SCM versus SCB. 


\subsection{In vitro characterization of new TFMEA interfaced to new SCB}

After characterization of the new SCB, it was interfaced with improved TFMEAs and tested using a new batch of conductive gel, in a similar manned as in section 5.2. Signals obtained from a group of five electrodes were displayed in Figure 5.16 and 5.17 and were representative of the quality of signals acquired from most TFMEA groups. Compared to the signals from section 5.2, a large improvement in signal quality was visible. As expected, the strange noise present with the SCM was no longer present. Also, it was noted that the signals contained a much lower DC offset than was seen previously.

Each channel of each TFMEA was verified on the conductive gel before it was considered for use in the in vivo experiment. Arrays which contained bad channels were not used in further experiments. Once the new SCB had been tested in conjunction with the improved TFMEAs and the system as a whole proved to be working well, it was able to be utilized for in vivo experimentation.

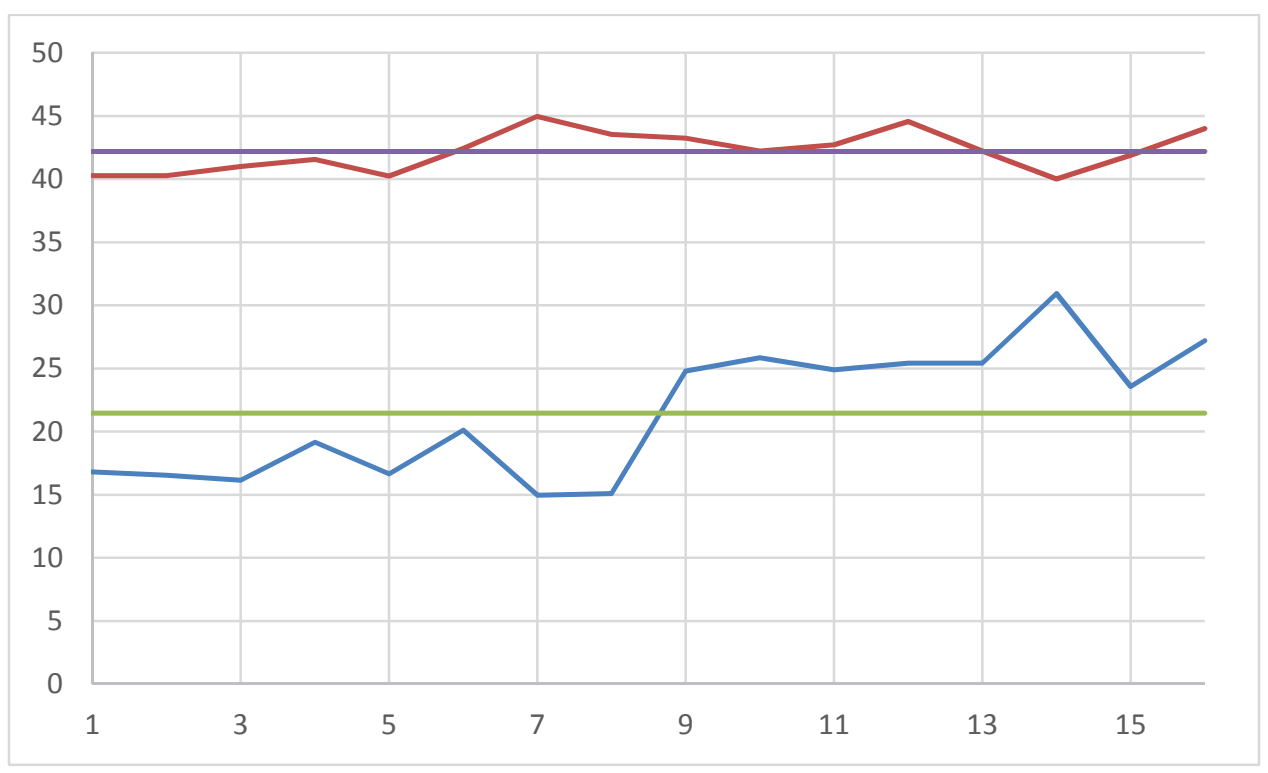

Figure 5.15 SNR of in vitro signals per channel. 
Figure 5.15 shows the signal to noise ratios of in vitro measurements taken with improved TFMEA on the conductive gel. The blue signal represents data obtained using the original SCM while the red signal represents data obtained using the new SCB. The green line shows the average SNR using the SCM, which was $21.5 \mathrm{~dB}$. The purple line shows the average SNR using the SCB, which was $42.2 \mathrm{~dB}$. Statistical analysis of this data is presented below.

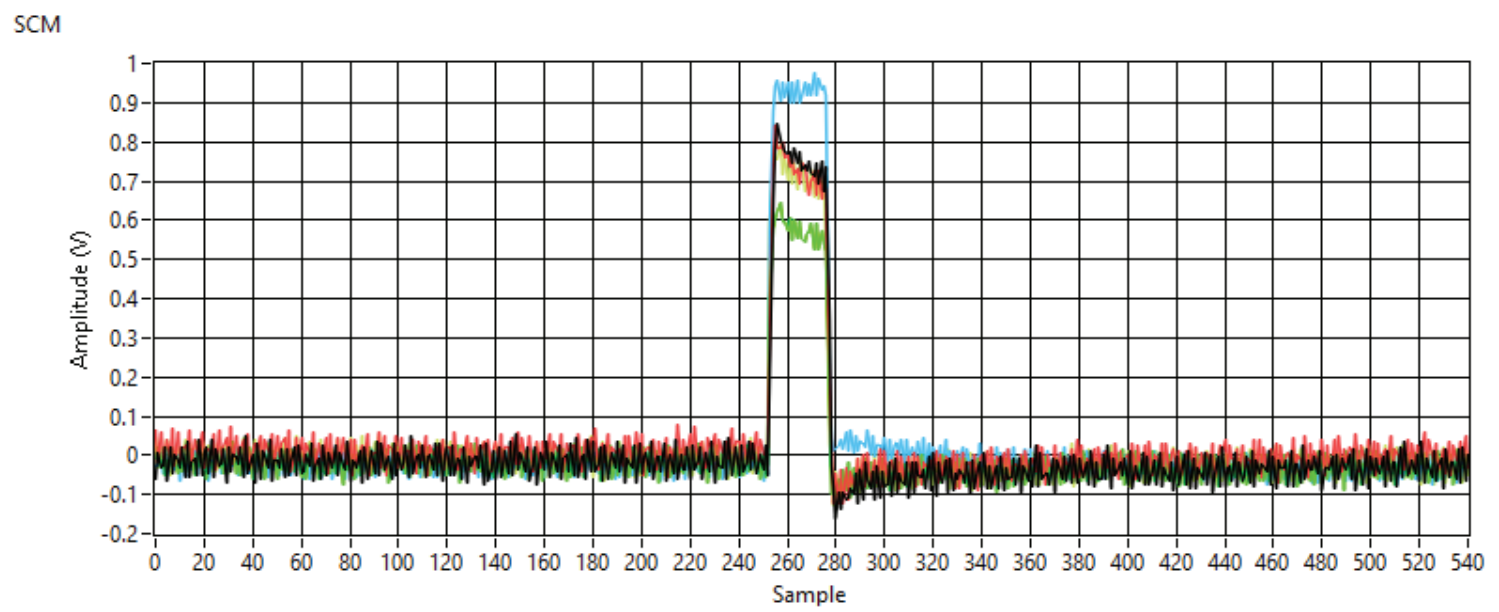

Figure 5.16 One group of five electrodes using improved TFMEA and SCM. $\mathrm{SCB}$

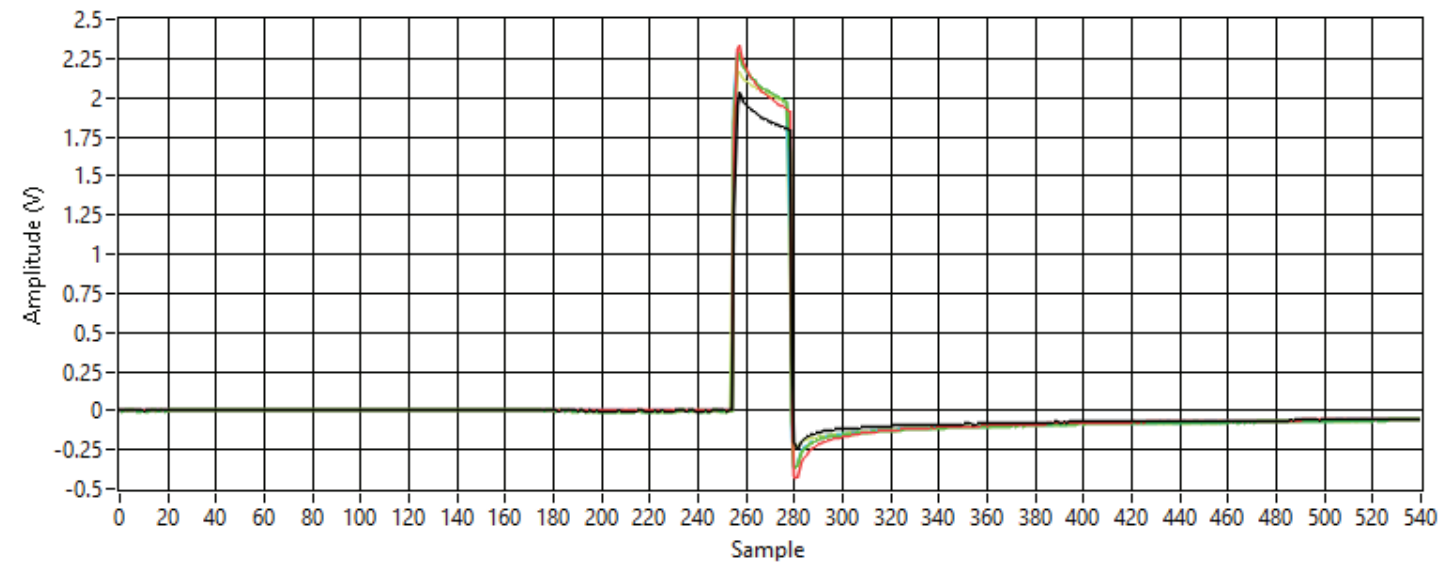

Figure 5.17 One group of five electrodes using improved TFMEA and SCB. 
Figure 5.16 shows a single pacing event from a single electrode group using the SCM. Figure 5.17 shows the same event using the SCB. Data was collected at $16 \mathrm{kHz}$ sample rate. Notice that the signal output from the SCB is much cleaner and has an overall greater amplitude compared to the output of the SCM.

\section{One-way ANOVA: C1 versus C2}
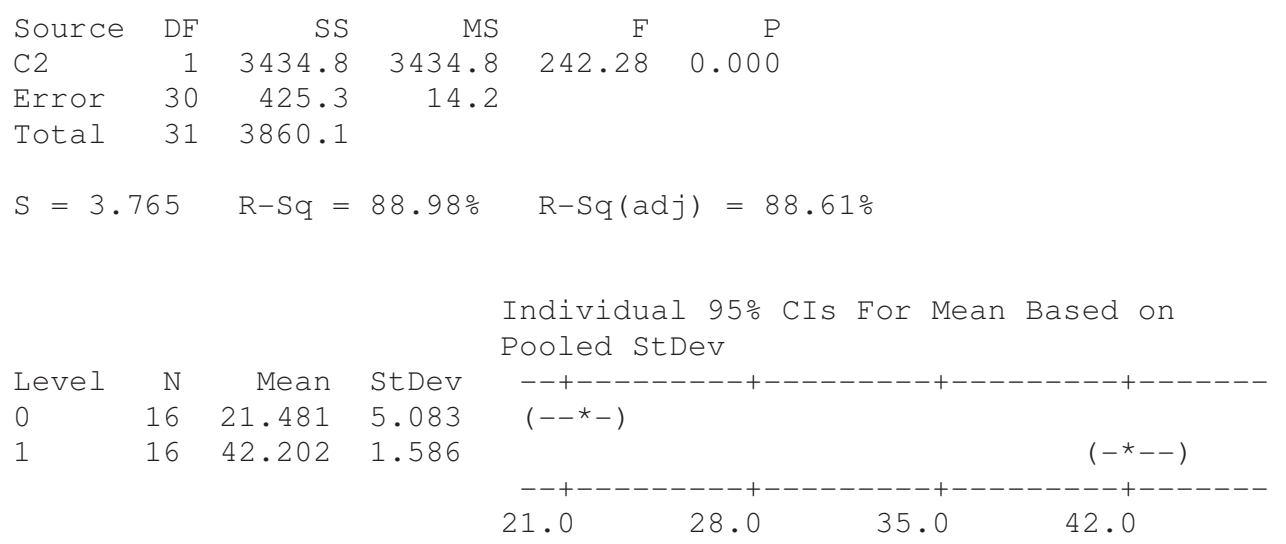

Pooled StDev $=3.765$

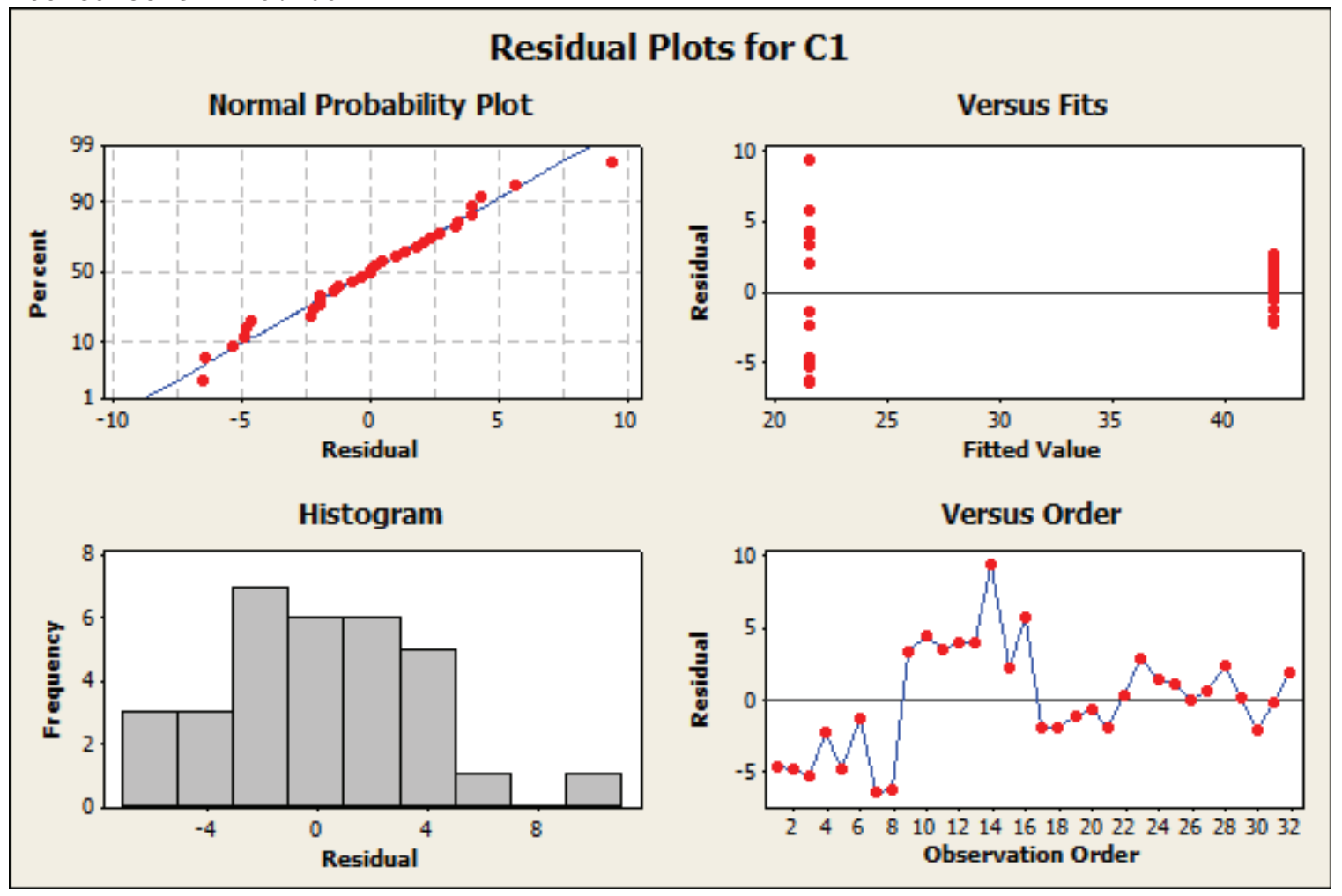

Figure 5.18 ANOVA plots of in vitro data generated from Minitab. 
A one way ANOVA was performed on the in vitro data using Minitab 16 with a confidence level of $95 \%$. The results indicated that there was a significant $(p=0.000)$ improvement in noise performance when using the new SCB and improved TFMEA versus the previous SCM. The R-squared value of $89 \%$ indicated a relatively good fit and the ANOVA plots shown in Figure 5.18 appear normal.

\subsection{In vivo characterization of new MEAs interfaced to new SCB}

Several months after the preliminary collection of data, a new batch of improved microelectrode arrays along with the new SCB were sent to the University of Utah. The following data was selected to be analyzed:

- dog, right ventricle, 20 seconds

- goat, right ventricle, 20 seconds

- goat, left ventricle, two 10 second runs

Data was collected at an $8 \mathrm{kHz}$ sample rate for all 25 channels using the new signal conditioning board. The first step in analyzing this data was to perform a fast Fourier transform (FFT) to determine where to set the high cutoff frequency for the software bandpass filter during data analysis. National Instruments LabVIEW was chosen to perform these FFTs, and to perform the data filtering. The FFT was computed from the data taken from the right ventricle of a dog in the frequency range of $0 \mathrm{~Hz}$ to $4 \mathrm{kHz}$ (which is the Nyquist limit of the $8 \mathrm{kHz}$ sampling rate). The FFT is shown in Figure 5.19 up to a frequency of $1.5 \mathrm{kHz}$. Beyond $1.5 \mathrm{kHz}$, there was little useful data compared to the actual signal, so this was chosen as the low-pass cutoff frequency. The high-pass cutoff frequency was chosen to be $0.5 \mathrm{~Hz}$, in order to filter out baseline drift. 


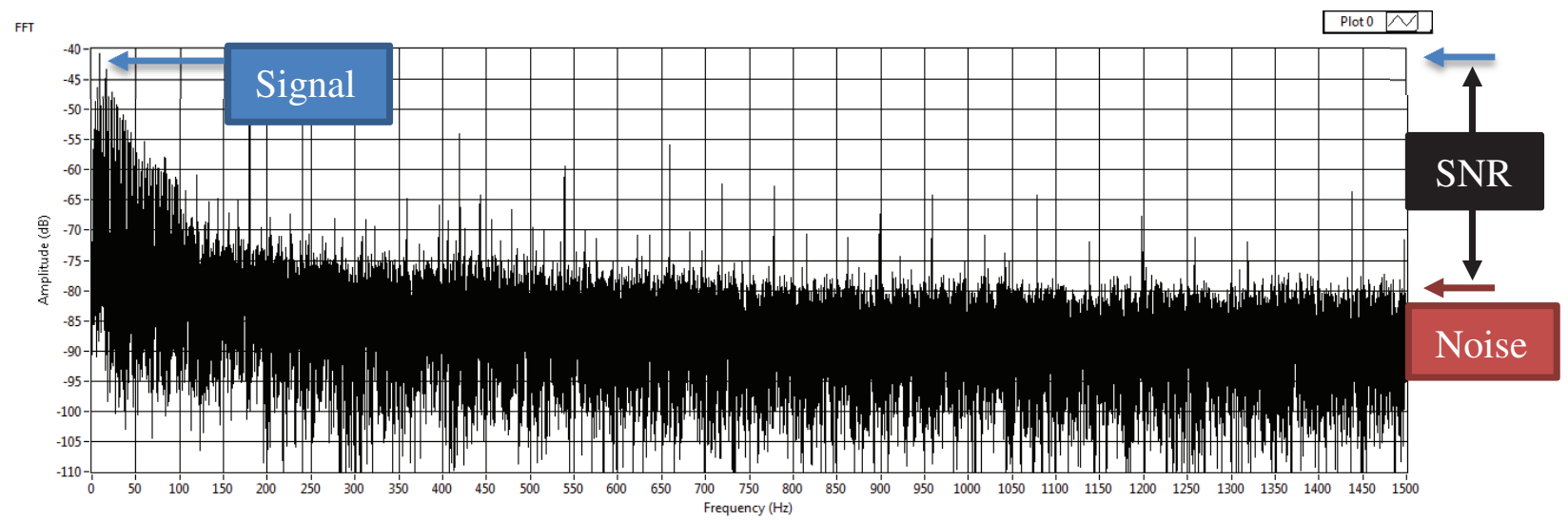

Figure 5.19 FFT from right ventricle data collected in a dog, 0 to $1.5 \mathrm{kHz}$. Signal level -40dB.

The signal level in figure 5.19 above was approximately $-40 \mathrm{~dB}$, while the noise level was $-80 \mathrm{~dB}$. This resulted in a signal to noise ratio of approximately $40 \mathrm{~dB}$. Important to note that the $-80 \mathrm{~dB}$ noise level was consistent with the noise floor measurements performed on the SCB alone (Figure 5.12). This shows that by connecting the TFMEA to the SCB, no additional noise was generated. A SNR of $40 \mathrm{~dB}$ from a dog heart was similar to that obtained in the original work by Dong, et al. However, the performance of the SCB alone remains superior to that of the SCM used previously.

In vivo recordings are highly susceptible to fabrication defects in the TFMEA. The conductive gel used in the in vitro experiments was stationary, so no movement artifacts were present in the recording. In the in vivo experiment the heart tissue was actively moving as the heart contracted and relaxed. This added to the demand placed on the electrodes, and, if there were fabrication errors they could manifest in the recorded data as noise. 
After the FFT was performed on each sample of data, the data was read into LabVIEW and filtered. For each of the data sets, the low-pass filter frequency used was the frequency identified from the FFT and the high-pass frequency was kept at $0.5 \mathrm{~Hz}$. A second order filter was chosen over a higher order filter to minimize phase shift as much as possible, which could affect interpretation of the SL. The filtered ECG data from

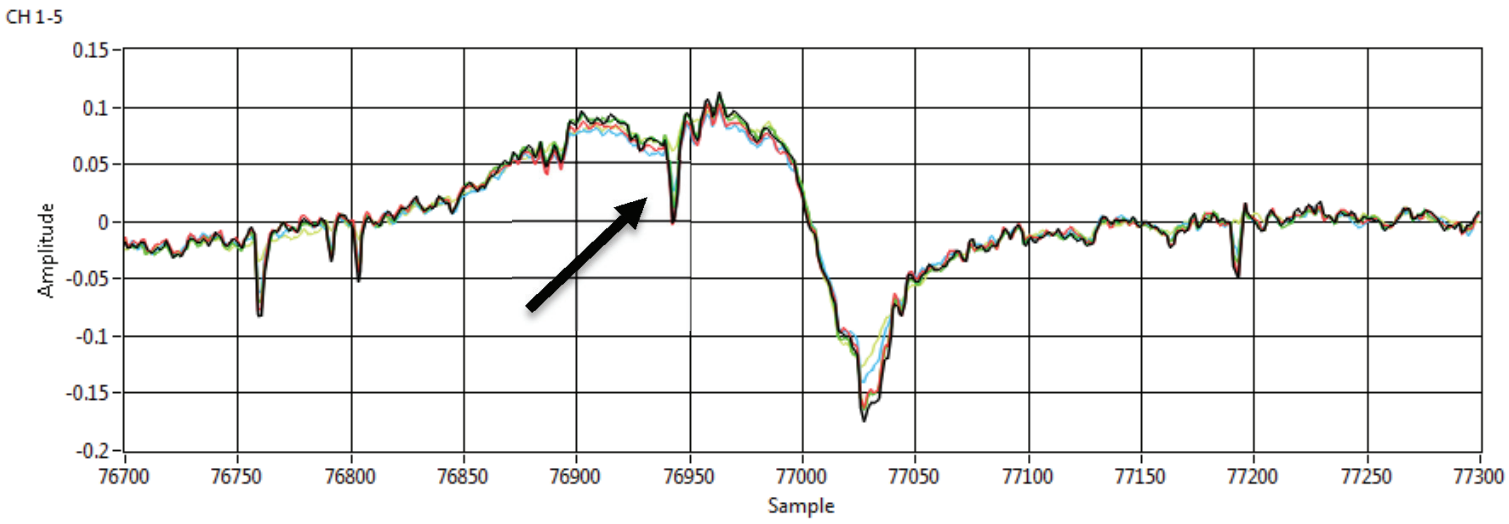

Figure 5.20 ECG data from dog, channels 1 to $5.2^{\text {nd }}$ order band-pass $0.5 \mathrm{~Hz}$ to 1.5 kHz.

channels 1-5 taken from the right ventricle of a dog heart is displayed in Figure 5.20. This group of channels was chosen because it illustrated the best example of the detail which the improved TFMEA-SCB system provided. Notice the black arrow pointing to a negative going slope in the ECG of Figure 5.20. Such detail is not normally seen in standard ECG recordings and represents detail from individual cardiomyocytes. If this data is filtered over the standard range for electrocardiograms $(\sim 0.5 \mathrm{~Hz}$ to $150 \mathrm{~Hz})$, this detail is almost completely lost. In addition, it would not have been visible at all at the surface of the body, where conventional ECGs are measured. 


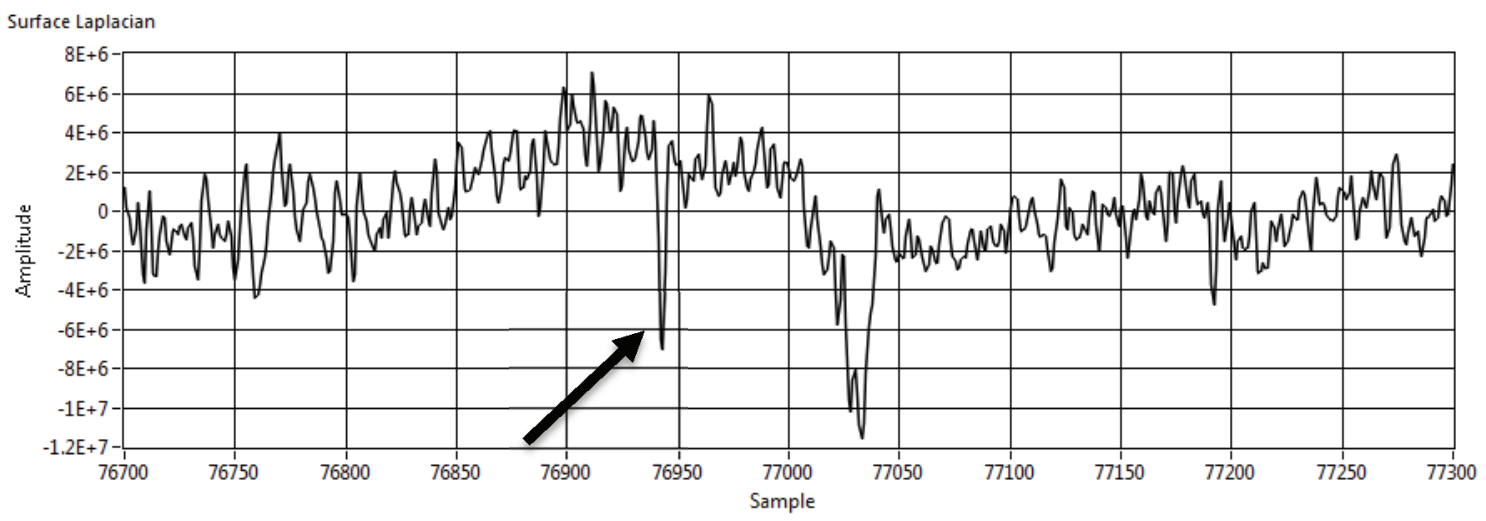

Figure 5.21 Surface Laplacian computed from the filtered data in Figure $\mathbf{5 . 1 2}$

The surface Laplacian of this group of five electrodes was also calculated from the filtered signals and is displayed in Figure 5.21. The black arrow on the SL signal corresponds to the feature in the ECG signals in Figure 5.20. The SL signal is useful in helping the researcher or clinician determine the moment of local tissue activation. This is often very important data to have during certain arrhythmias, especially ventricular fibrillation. During a VF event, cardiomyoctes are being activated all throughout the heart in a disconcerted fashion. Because of this, the resultant VF ECG signal is a summation of many different signals coming together at one point, making it nearly impossible to determine the moment when a localized region of heart tissue is being activated. Unfortunately, no VF events were recorded during either in vivo study. SL signals derived from normal sinus rhythm ECGs are not as useful as those derived from ECGs during an event such as VF. However, deriving the SL from the available data recorded with the 
improved TFMEA and SCB system proves that it is feasible to use the system during more complex events and useful information could be acquired from the SL.

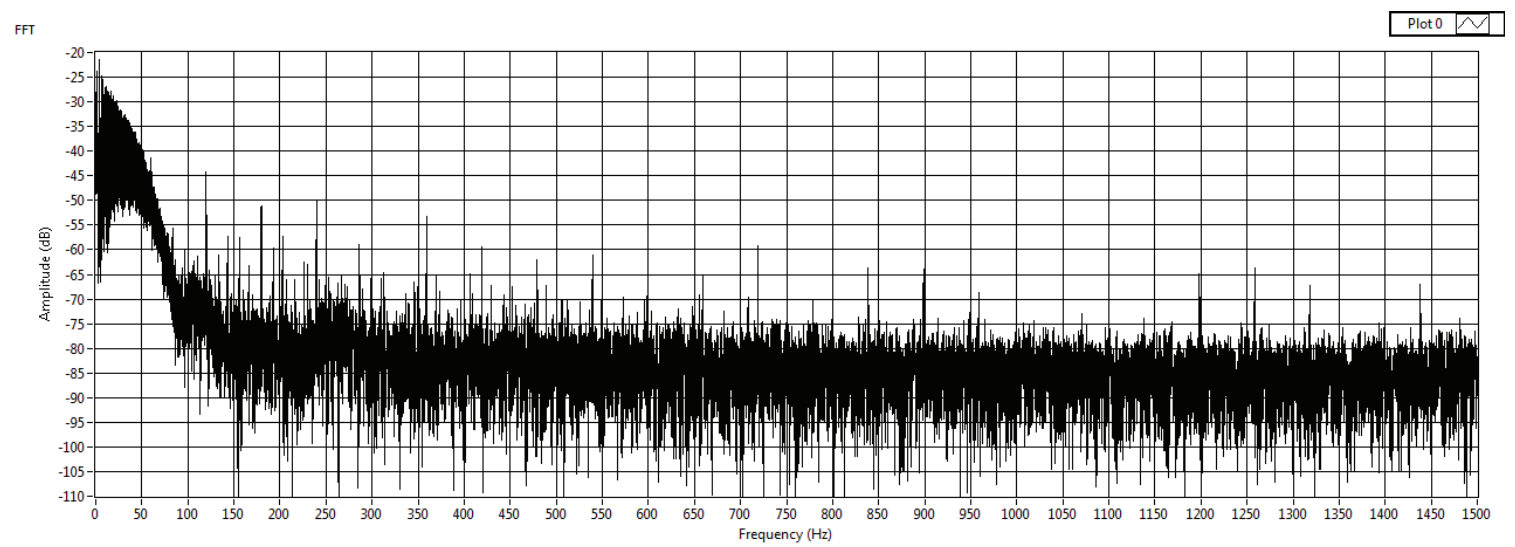

Figure 5.22 FFT from left ventricle data collected in a goat run 2,0 to $1.5 \mathrm{kHz}$. Signal level -20 dB.

From the FFT in Figure 5.22 it was shown that the signal level from the first data collected from the left ventricle of the goat had an amplitude of $-20 \mathrm{~dB}$. The noise level is approximately $-75 \mathrm{~dB}$, making the SNR for this group of data $55 \mathrm{~dB}$. Notice that compared to the right ventricle of the dog, the signal acquired from the goat was greater in amplitude. This could be because goats are slightly larger and produce a larger amplitude electrocardiogram. Because of this increase in amplitude the SNR increased considerably. A SNR of $55 \mathrm{~dB}$ is considerably higher than what was achieved in previous work, about $10 \mathrm{~dB}$ on average. 


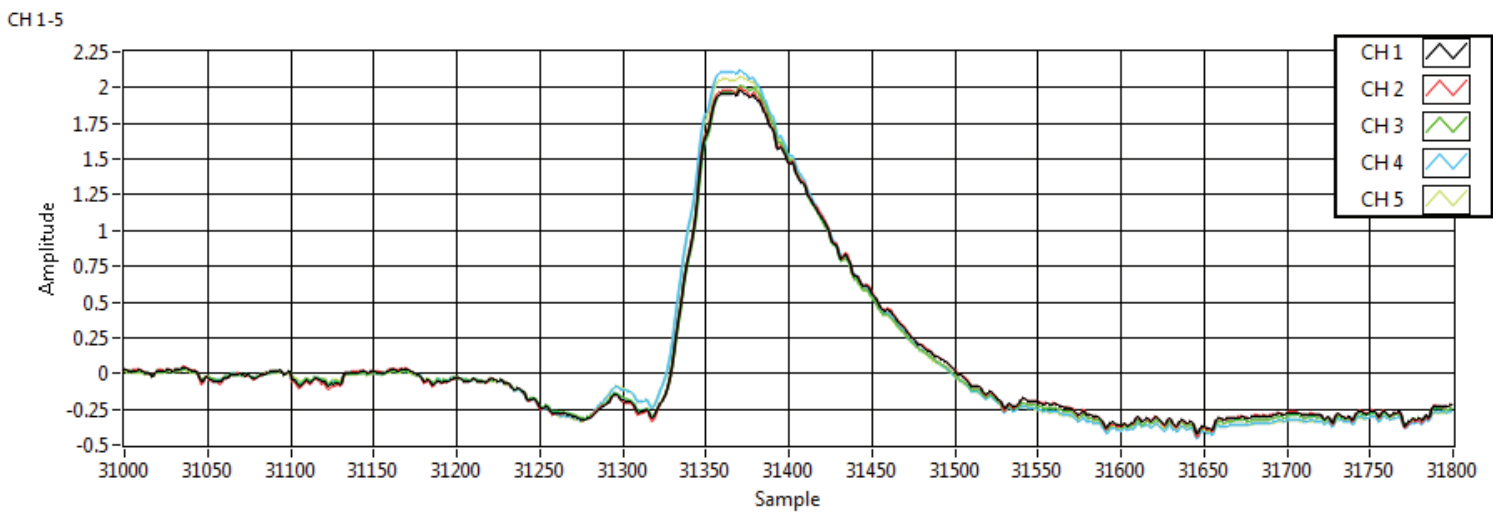

Figure 5.23 ECG from goat left ventricle run 2, channels 1 to $5.2^{\text {nd }}$ order band-pass $0.5 \mathrm{~Hz}$ to $1.5 \mathrm{kHz}$.

Surface Laplacian $\mathrm{CH} 1-5$

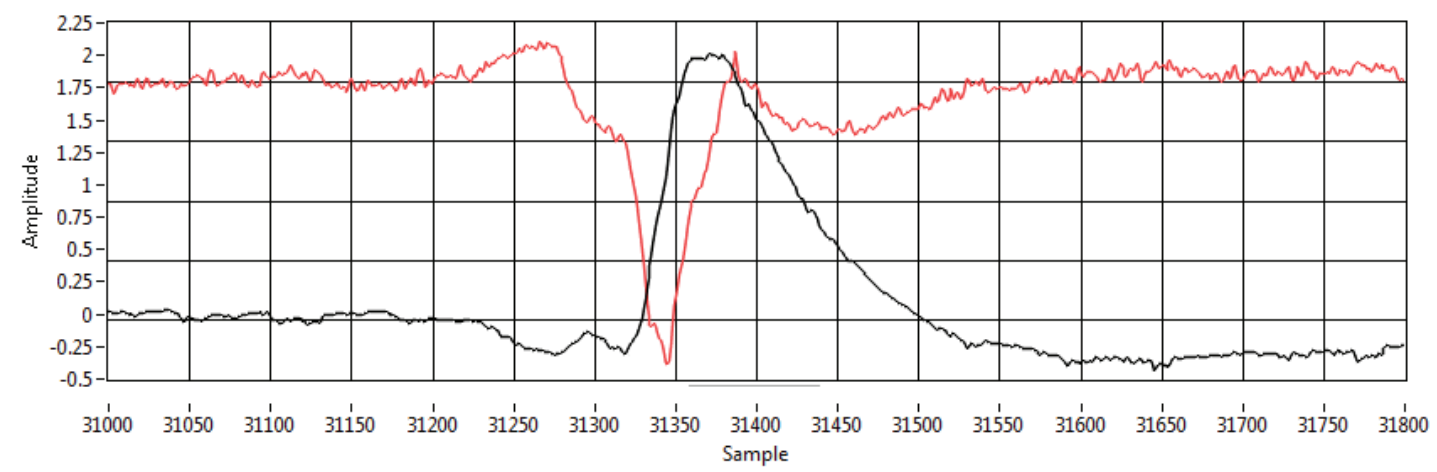

Figure 5.24 Surface Laplacian from data in Figure 5.15

The filtered ECG is shown in Figure 5.23 and the SL derived from these signals is shown in Figure 5.24. The data was band-pass filtered from $0.5 \mathrm{~Hz}$ to $1.5 \mathrm{kHz}$ using a $2^{\text {nd }}$ order filter. The black line in Figure 5.24 indicated the ECG signal from the center electrode from group 1-5 and the red line indicated the resulting SL electrogram. The most negative portion of the SL, to the left of sample 31350, indicated the moment of local tissue activation at the center electrode. Both the ECG and resulting SL electrograms are very clean and readable, owing to the reduction in electrode resistance and the performance of the new SCB. 
Compared to the preliminary in vivo data that used the TFMEA-SCM system it was shown that the TFMEA-SCB system provided a performance improvement. The SNR of recordings made with the previous SCM system were approximately $22 \mathrm{~dB}$. With such a high amount of noise it was not possible to achieve the level of detail required for more complex measurements such as the SL. An electrocardiogram recording with SNR of 40 dB was previously achieved by Dong, et al and shown to be adequate for SL calculation. With the TFMEA-SCB system, SNR as high as $55 \mathrm{~dB}$ was achieved with data from the left ventricle of a goat heart. 


\section{CONCLUSION AND RECOMMENDATION}

Electrode resistance was reduced by an order of magnitude, from $1000 \Omega$ to $100 \Omega$, with the addition of a $200 \mathrm{~nm}$ layer of silver to the metal traces. Issues with a previously developed signal conditioning microchip prompted the development of a new signal conditioning board which achieved low noise, low DC offset, and high gain performance. The reduction of TFMEA electrode resistance in conjunction with the development of a robust, low noise signal conditioning board was shown to significantly increase total signal to noise ratio of signals recorded in vitro, compared with results obtained previously by our group.

The current TFMEA design provides resolution at the cellular level. However, fabrication was quite difficult and required considerable microfabrication experience. Each TFMEA was extremely fragile and easily damaged. More work should be done to increase the durability of the overall sensor as well as the electrode. Considerable challenges were presented during MEMS silver electroplating. It would be well worth the effort to develop an array which is not so critically dependent on this step.

The SCB could be redesigned to be modular, which would allow it to be scalable. It could be used with MEAs of differing channel count and specifications, such as for neural recordings. This could be achieved by adding a header between two boards which would provide power. It may also be possible to power the SCB from the data acquisition lines instead of using a battery, this would reduce the size of the board and make it easier to use

during in vivo studies. An SCB which has integrated analog to digital conversion would also provide a large improvement in signal quality by being much more immune to noise. 
Modifications to the design and fabrication method of the TFMEA would enable greater experimentation. It required months to fabricate functional TFMEAs and if this were reduced to one week it would be possible to test many different designs over the course of a project. In combination with TFMEA improvements, increasing modularity and signal quality of the SCB will enhance the extraction of information from biological systems which is useful to researchers as well as clinicians. 


\section{REFERENCES}

1. Go, Alan S., et al. 2013. Heart Disease and Stroke Statistics - 2013 Update A Report From the American Heart Association. Circulation. 127:161.

2. Stett, A., et al. 2003. Biological application of microelectrode arrays in drug discovery and basic research. Anal Bioanal Chem. 377(3):486-95.

3. Coronel, R., et al. 2000. Laplacian electrograms and the interpretation of complex ventricular activation patterns during ventricular fibrillation. $J$ Cardiovasc Electrophysiol. 11(10):1119-28.

4. Heim, M., et al. 2012. Combined macro-/mesoporous microelectrode arrays for low-noise extracellular recording of neural networks. J Neurophysiol. 108(6):17931803.

5. Jones, I. L., et al. 2011. The potential of microelectrode arrays and microelectronics for biomedical research and diagnostics. Anal Bioanal Chem. 399(7):2313-2329.

6. Dong, H. 2011. Design, Characterization And Testing Of A Thin-Film Microelectrode Array And Signal Conditioning Microchip For High Spatial Resolution Surface Laplacian Measurement. Department of Electrical Engineering Ph.D., University of Louisville.

7. Kim, S., et al. 2004. Use of micro machined probes for the recording of cardiac electrograms in isolated heart tissues. Biosensors and Bioelectronics. 19(9):11091116.

8. Jacquir, S., et al. 2008. Cardiac arrhythmias induced by an electrical stimulation at a cellular level in Computers in Cardiology, 2008.

9. Frey, U., et al. 2010. Switch-Matrix-Based High-Density Microelectrode Array in CMOS Technology. IEEE Journal of Solid-State Circuits. 45(2):467-482.

10. Xin, Z., et al. 2011. Development of planar microelectrode array for extracellular characterization of action potentials in sinoatrial node cells. 2011 International Symposium on Bioelectronics and Bioinformatics (ISBB).

11. Layer, H. et al. 1962. Effects of Quenching and Annealing on Ionic Conductivity and on Dislocation Decorability in Silver Chloride. Journal of Applied Physics. 33(1):478-481. 
12. Göktepe, Serdar, et al. 2010. A multiscale model for eccentric and concentric cardiac growth through sarcomerogenesis. Journal of Theoretical Biology. 265(3):433-442.

13. Comparison between a positive tone resist and a negative tone resist. Available from: http://en.wikipedia.org/wiki/Photoresist.

14. Whitesides, George M., et al. 1997. Microcontact Printing and Electroplating on Curved Substrates: Production of Free-Standing Three-Dimensional Metallic Microstructure. Advanced Materials. 9(6):475-477. 


\section{VITA}

Mr. Christopher Hayward Osborne received his B.S. in Bioengineering from the University of Louisville in 2010 and has since pursued a graduate degree in the same field. He has developed advanced microfabriaction skills during time spent in the University's class 100 cleanroom as well as developed extensive knowledge of analog electronics, especially in the field of bioelectronics. Engineering in the field of audio systems has always been a passion for him and he wishes to pursue it further once completing his graduate degree. Mr. Osborne currently resides in Louisville, KY. 